\title{
PLANAR ANTENNAS IN LTCC TECHNOLOGY FOR ULTRA-WIDEBAND APPLICATIONS
}

\author{
By \\ Grzegorz M. Brzezina, B.Eng.
}

\author{
A thesis \\ presented to Carleton University \\ in fulfilment of the \\ thesis requirement for the degree of \\ MASTER OF APPLIED SCIENCE \\ in \\ ELECTRICAL ENGINEERING
}

\author{
Ottawa, Ontario, Canada \\ (C) Copyright 2005 \\ Grzegorz M. Brzezina
}




$\begin{array}{ll}\begin{array}{l}\text { Library and } \\ \text { Archives Canada }\end{array} & \begin{array}{l}\text { Bibliothèque et } \\ \text { Archives Canada }\end{array} \\ \begin{array}{l}\text { Published Heritage } \\ \text { Branch }\end{array} & \begin{array}{l}\text { Direction du } \\ \text { Patrimoine de l'édition }\end{array} \\ \begin{array}{l}\text { 395 Wellington Street } \\ \text { Ottawa ON K1A ON4 }\end{array} & \begin{array}{l}\text { 395, rue Wellington } \\ \text { Ottawa ON K1A ON4 } \\ \text { Canada }\end{array}\end{array}$

Your file Votre référence

ISBN: 0-494-06785-3

Ourfile Notre référence

ISBN: 0-494-06785-3

NOTICE:

The author has granted a nonexclusive license allowing Library and Archives Canada to reproduce, publish, archive, preserve, conserve, communicate to the public by telecommunication or on the Internet, loan, distribute and sell theses worldwide, for commercial or noncommercial purposes, in microform, paper, electronic and/or any other formats.

The author retains copyright ownership and moral rights in this thesis. Neither the thesis nor substantial extracts from it may be printed or otherwise reproduced without the author's permission.
AVIS:

L'auteur a accordé une licence non exclusive permettant à la Bibliothèque et Archives Canada de reproduire, publier, archiver, sauvegarder, conserver, transmettre au public par télécommunication ou par l'Internet, prêter, distribuer et vendre des thèses partout dans le monde, à des fins commerciales ou autres, sur support microforme, papier, électronique et/ou autres formats.

L'auteur conserve la propriété du droit d'auteur et des droits moraux qui protège cette thèse. $\mathrm{Ni}$ la thèse ni des extraits substantiels de celle-ci ne doivent être imprimés ou autrement reproduits sans son autorisation.
In compliance with the Canadian

Privacy Act some supporting forms may have been removed from this thesis.

While these forms may be included in the document page count, their removal does not represent any loss of content from the thesis.
Conformément à la loi canadienne sur la protection de la vie privée, quelques formulaires secondaires ont été enlevés de cette thèse.

Bien que ces formulaires aient inclus dans la pagination, il n'y aura aucun contenu manquant.

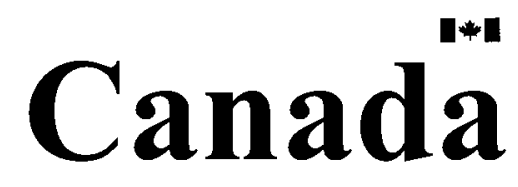




\section{Abstract}

This thesis investigates the feasibility of designing and fabricating small, low cost, ultrawideband (UWB) compliant antennas. UWB uses very short and low power pulses with precise timing to transmit data at high rates within the $3.1 \mathrm{GHz}$ to $10.6 \mathrm{GHz}$ band. These features have created the need to evaluate antennas in both the frequency and time domain for use in UWB systems.

The need for practical design guidelines for UWB antennas is addressed. Antenna designs based on these guidelines are presented and realizations in both FR-4 and low temperature co-fired ceramic (LTCC) technology are demonstrated. A finite difference time domain (FDTD) based electromagnetic simulator is developed, allowing the time domain response of UWB antennas to be studied under arbitrary pulse shape excitations. Its results are compared with a commercial FEM-based electromagnetic solver, and both tools are employed in the design of several candidate UWB antenna structures.

Given their planar topology, circuit integration possibilities and compact size (no larger than $7 \mathrm{~cm}$ on one side), antipodal Vivaldi antennas (AVA) and partial ground plane triangular monopole antennas (PGP-TM) are fully characterized.

The AVA constructed on an FR-4 substrate has a measured impedance bandwidth of 
$6 \mathrm{GHz}$, from $4 \mathrm{GHz}$ to $10 \mathrm{GHz}$. Radiation pattern measurements, performed in an anechoic chamber, revealed a directional pattern with a gain of $4.32 \mathrm{~dB}$ at $6 \mathrm{GHz}$. However, at $8 \mathrm{GHz}$ pattern stability degraded because of the limitations of the FR-4 substrate at high frequencies. The LTCC version of the AVA shows no such deficiencies while occupying $55 \%$ less area than its FR-4 counterpart. An increasing system $\left|S_{21}\right|$ slope of $19 \mathrm{~dB}$ per decade is observed - making this the only antenna in this study that provides a suitable cancelation for path loss. This antenna also incorporates a novel circuit feature to accommodate transceiver electronics. The impedance bandwidth for this antenna was 3.35 GHz, from $6.65 \mathrm{GHz}$ to $10 \mathrm{GHz}$. This makes it suitable for use in the upper band of the direct sequence (DS) UWB implementation. Gain was measured to be $5 \mathrm{~dB}$ at $8 \mathrm{GHz}$. A UWB pulse was transmitted and received with very little added distortion in an antenna system utilizing these AVAs.

Omni-directional antennas were also designed and fully characterized. Beginning with an orthogonal ground plane triangular monopole (OGP-TM) that has a bandwidth of 1.14 GHz from $4.5 \mathrm{GHz}$ to $5.64 \mathrm{GHz}$. This antenna has a gain of $0 \mathrm{~dB}$ and a monopole like pattern. This antenna shows that broadband antennas can be designed using very high permittivity substrates of $\varepsilon_{r}=68$. The characteristics of this antenna make it suitable for many wireless local area network applications. Planar omni-directional antennas were also investigated. The PGP-TM based on a FR-4 substrate has a return loss bandwidth of $3.35 \mathrm{GHz}$ or $4.9 \mathrm{GHz}$ when a small peak above $-10 \mathrm{~dB}$ at $8 \mathrm{GHz}$ is neglected - making this antenna nearly capable of spanning the full DS-UWB bandwidth. The expected decreasing system $\left|S_{21}\right|$ of almost $20 \mathrm{~dB}$ per decade is observed. The PGP-TM implemented in LTCC 
occupies $29 \%$ less area than its FR-4 counterpart while also providing better radiation and time domain performance. The impedance bandwidth is measured to be from $3.57 \mathrm{GHz}$ to $6.53 \mathrm{GHz}$ - making this antenna suitable for use in the lower band of the DS-UWB implementation. The slope of the system $\left|S_{21}\right|$ is the same as with the FR-4 PGP-TM. Both substrate implementations of the PGP-TM antenna utilize novel parasitic tuning elements for optimized performance.

The measurement results, which are in good agreement with the simulations, indicate that the antennas based on LTCC deliver similar or superior performance compared to their FR-4 counterparts, while having the advantages of smaller size and increased packaging possibilities. Improvements to the FDTD software, including upgrading absorbing boundary conditions and adding radiation pattern generation capability, are recommended as future work. 


\section{Acknowledgements}

I would like to thank Professor Langis Roy for his advice and supervision throughout this thesis work; Professor Len MacEachern for his advice and for providing me with valuable computer resources; Nagui Mikhail for his technical support; Atif Shamim for his help with the anechoic chamber; Michael Mariani for his help with HFSS and Yasser Soliman for sharing his knowledge of Latex. Many thanks to you all.

I would like to recognize the support of the Communications Research Center, VTT in Finland and Carleton University for providing the research and fabrication facilities. The financial support received from Nortel Networks is gratefully acknowledged.

A am grateful to my mother and sister for supporting me all this time. This thesis would not be possible without you. Finally, I wish to dedicate this thesis to the memory of my father. Thank you for inspiring me so much. 


\section{Table of Contents}

$\begin{array}{ll}\text { Abstract } & \text { iii }\end{array}$

$\begin{array}{lll}\text { Acknowledgements } & \text { vi }\end{array}$

$\begin{array}{ll}\text { Table of Contents } & \text { vii }\end{array}$

List of Tables $\quad$ xi

List of Figures $\quad$ xiii

1 Introduction $\quad 1$

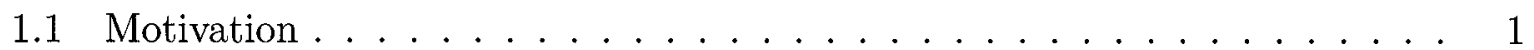

1.2 Thesis Goals . . . . . . . . . . . . . . . . . . . . 3

1.3 Thesis Outline ....................... . . 4

2 Technology Overview $\quad 7$

2.1 About UWB . . . . . . . . . . . . . . . . . 7

2.2 History of UWB . . . . . . . . . . . . . . 8

2.3 Two flavors of UWB . . . . . . . . . . . . . . . . 9 
2.4 Important Antenna Parameters . . . . . . . . . . . . . . . . . 12

2.4.1 Radiation Pattern . . . . . . . . . . . . . . 12

Radiation Pattern Lobes . . . . . . . . . . . . . . 13

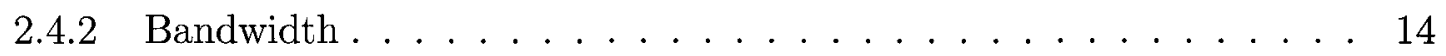

Impedance Bandwidth . . . . . . . . . . . . . . . 14

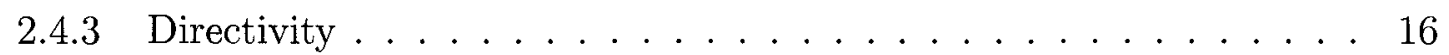

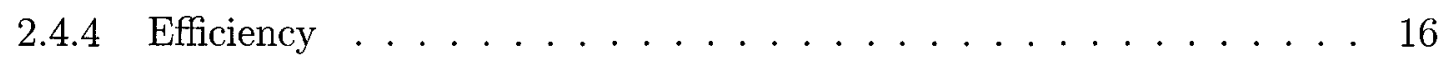

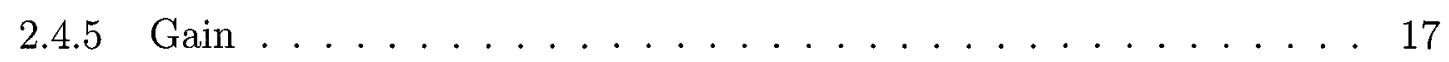

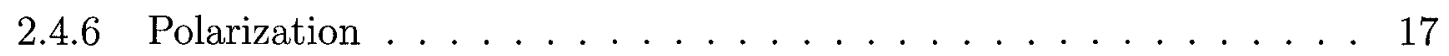

2.5 Low Temperature Co-fired Ceramic RF Modules . . . . . . . . . . . . . 18

2.6 A Critical Overview of Current UWB Antenna Requirements . . . . . . . . 21

2.6.1 UWB Antenna Requirements . . . . . . . . . . . . . . 21

2.6.2 Directivity and System Performance . . . . . . . . . . . . 23

2.7 UWB Principles . . . . . . . . . . . . . . . . . 25

2.7 .1 UWB Antenna Guidelines . . . . . . . . . . . . . . . 31

2.8 Conclusion . . . . . . . . . . . . . . . . 32

3 The Finite Difference Time Domain (FDTD) Technique 33

3.1 Introduction to Maxwell's Equations and the Yee Algorithm . . . . . . . . 33

3.2 Numerical Stability and Dispersion . . . . . . . . . . . . . 39

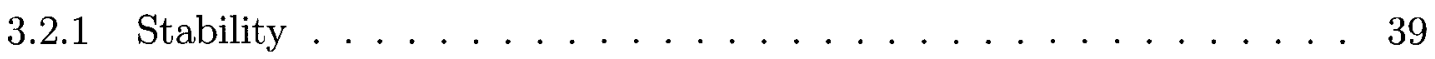

viii 
3.2 Dispersion ...................... 40

3.3 Excitation Methods . . . . . . . . . . . . . . . . 40

3.4 Absorbing Boundary Conditions . . . . . . . . . . . . . . . 43

3.5 Conductor and Media Interface Treatment . . . . . . . . . . . . . . 44

3.6 Software Development and Antenna Modeling . . . . . . . . . . . . 45

3.6.1 Near Field Probing .................. 51

3.7 Conclusion . . . . . . . . . . . . . . . . . . 52

4 Practical UWB Antenna Design $\quad 54$

4.1 Vivaldi Antenna Theory . . . . . . . . . . . . . . 55

4.2 Antipodal Vivaldi Antenna Design and Simulation . . . . . . . . . . . 58

4.2 .1 FDTD Source Excitation . . . . . . . . . . . . 62

4.2.2 FR-4 based Antipodal Vivaldi Simulation . . . . . . . . . . . . 62

4.2.3 LTCC-based antipodal Vivaldi simulation . . . . . . . . 66

4.3 Balanced Antipodal Vivaldi Antenna Design and Simulation . . . . . . . . 70

4.3.1 LTCC-based Balanced Antipodal Vivaldi Simulation . . . . . . . 72

4.4 UWB Monopole Antenna Theory . . . . . . . . . . . . . . . . 74

4.5 Partial Ground Plane Triangular Monopole Antenna Design and Simulation 76

4.5.1 FR-4 Based Partial Ground Plane Triangular Monopole Simulation 79

4.5.2 LTCC-based Partial Ground Plane Triangular Monopole Simulation 83 
4.6 Triangular Monopole Antenna with Orthogonal Ground Plane Design and Simulation . . . . . . . . . . . . . . . . 87

4.6.1 Orthogonal Ground Plane Triangular Monopole Simulation . . . . . 90

4.7 Planar Circuit Integration . . . . . . . . . . . . . . 91

4.8 Simulation Summary . . . . . . . . . . . . . . . . . 93

5 UWB Antenna Measurements and Comparisons $\quad 95$

5.1 Measurement Setup . . . . . . . . . . . . . . . 95

$5.25 \mathrm{GHz}$ Series Patch Array . . . . . . . . . . . . . . 98

5.3 Orthogonal Ground Plane Triangular Monopole . . . . . . . . . . . . . 99

5.4 FR4-based Antipodal Vivaldi . . . . . . . . . . . . . . . 102

5.5 FR-4 based Triangular Monopole with Partial Ground Plane . . . . . . . . 106

5.6 LTCC-based Antipodal Vivaldi . . . . . . . . . . . . . 110

5.7 LTCC-based Triangular Monopole with Partial Ground Plane . . . . . . . 115

5.8 Measurement Summary and Discussion . . . . . . . . . . . . . 119

6 Conclusions and Future Work $\quad 124$

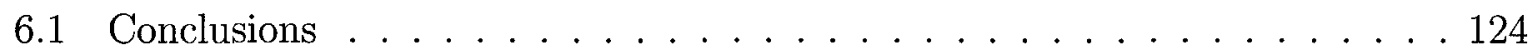

6.2 Main Contributions . . . . . . . . . . . . . . . . . 127

6.3 Future Work . . . . . . . . . . . . . . . 128

Bibliography 


\section{List of Tables}

2.1 Radiation Pattern Properties .................. . 14

2.2 Polarization States . . . . . . . . . . . . . . . . . 18

2.3 LTCC Tape Material Data . . . . . . . . . . . . . . . . . . 20

2.4 UWB Antenna Requirements . . . . . . . . . . . . . . . . . 22

2.5 Gain and Link Performance for Electrically Small Antennas . . . . . . . . 25

3.1 Sheen Patch Space Steps . . . . . . . . . . . . . . . . . 49

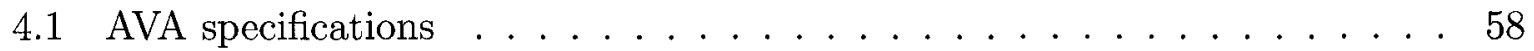

4.2 FR-4 AVA design parameters . . . . . . . . . . . . . . 61

4.3 LTCC AVA specifications . . . . . . . . . . . . . . 61

4.4 LTCC AVA design parameters . . . . . . . . . . . . . . 62

4.5 FR-4 based AVA FDTD analysis parameters . . . . . . . . . . . 63

4.6 Simulated FR-4 AVA radiation pattern properties . . . . . . . . . . 65

4.7 LTCC based AVA FDTD analysis parameters . . . . . . . . . . . 67

4.8 Simulated LTCC AVA radiation pattern properties . . . . . . . . . . 69

4.9 LTCC B-AVA design parameters . . . . . . . . . . . . . . 72

4.10 Simulated LTCC BAVA radiation pattern properties . . . . . . . . . . . 74 
4.11 FR-4 based PGP-TM specifications . . . . . . . . . . . . . . 77

4.12 PGP-TM dimensions . . . . . . . . . . . . . . . . . 78

4.13 FR-4 based PGP-TM FDTD analysis parameters . . . . . . . . . . 79

4.14 LTCC-based PGP-TM FDTD analysis parameters . . . . . . . . . . . . . 83

4.15 OGP-TM Specifications ...................... 88

4.16 OGP-TM dimensions . . . . . . . . . . . . . . . . . 89

5.1 Radiation pattern results summary for FR-4 based AVA . . . . . . . . 105

5.2 LTCC-based AVA radiation pattern results comparison . . . . . . . . . 114

5.3 UWB Antenna Measurement Summary . . . . . . . . . . . . . . . 123 


\section{List of Figures}

2.1 UWB Network $\ldots \ldots \ldots \ldots \ldots \ldots$

2.2 FCC Indoor Spectrum Mask . . . . . . . . . . . . . . . 10

2.3 OFDM Bands . . . . . . . . . . . . . . . . . . . . 10

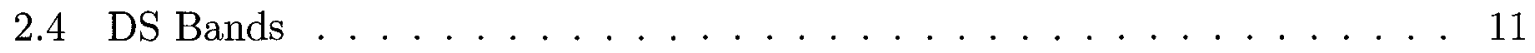

2.5 3-D LTCC Structure . . . . . . . . . . . . . . . . . . . . . . . 19

2.6 Double ridged horn antenna $\ldots \ldots \ldots \ldots \ldots \ldots$

2.7 Helical antenna . . . . . . . . . . . . . . . . . . . . 28

2.8 Equiangular spiral antenna . . . . . . . . . . . . . . . . . . . 29

2.9 Equiangular spiral antenna . . . . . . . . . . . . . . . . . . . . . . 29

2.10 Sierpinski monopole . . . . . . . . . . . . . . . . . . . . 31

3.1 The FDTD unit cell $\ldots \ldots \ldots \ldots \ldots \ldots$

3.2 Broadband Gaussian Excitation . . . . . . . . . . . . . . . 41

3.3 Microstrip Excitation . . . . . . . . . . . . . . . . 42

3.4 FDTD program execution $\ldots \ldots \ldots \ldots \ldots \ldots$

3.5 FDTD E field code segment $\ldots \ldots \ldots \ldots \ldots$

3.6 FDTD Mur ABC code segment $\ldots \ldots \ldots \ldots$. . . . . . . . 47

xiii 
3.7 FDTD $\mathbf{H}$ field code segment . . . . . . . . . . . . . . . 47

3.8 Rectangular microstrip patch antenna . . . . . . . . . . . . . . 48

3.9 Time step: $200 \ldots \ldots \ldots \ldots$

3.10 Time step: $200 \ldots \ldots$. . . . . . . . . . . . . . . . 49

3.11 Timestep: $400 \ldots \ldots \ldots \ldots \ldots \ldots$

3.12 Time step: $400 \ldots \ldots \ldots \ldots \ldots \ldots$

3.13 Time step: $800 \ldots \ldots \ldots \ldots \ldots$

3.14 Time step: $800 \ldots \ldots \ldots \ldots \ldots$. . . . . . . . . . . . 50

3.15 Simulation Results for Patch Antenna . . . . . . . . . . . . . . . 51

3.16 Simulated near field probe result for patch antenna . . . . . . . . . . 52

4.1 Tapered slot Vivaldi with Marchand balun feed . . . . . . . . . . . 55

4.2 Antipodal Vivaldi antenna topology (microstrip feed) . . . . . . . . . 56

4.3 Balanced antipodal Vivaldi antenna topology (stripline feed) . . . . . . . 56

4.4 Response in the time domain of (a) log-periodic and (b) antipodal Vivaldi





4.6 Simulated FR-4 AVA return loss . . . . . . . . . . . . . . 63

4.7 Simulated FR-4 based AVA 3-dimensional radiation pattern . . . . . . 64

4.8 Simulated FR-4 AVA 4 GHz XY-plane . . . . . . . . . . . . . . 64

4.9 Simulated FR-4 AVA 4 GHz XZ-plane . . . . . . . . . . . . . . . 64

4.10 Simulated FR-4 AVA 8 GHz XY-plane . . . . . . . . . . . 65

4.11 Simulated FR-4 AVA 8 GHz XZ-pane . . . . . . . . . . . . 65 xiv 
4.12 Simulated near field probe result for FR-4 AVA . . . . . . . . . . 66

4.13 Simulated LTCC AVA return loss . . . . . . . . . . . . . . 67

4.14 Simulated LTCC AVA $6 \mathrm{GHz}$ XY-plane . . . . . . . . . . . . . . 68

4.15 Simulated LTCC AVA $6 \mathrm{GHz}$ XZ-plane . . . . . . . . . . . . . 68

4.16 Simulated LTCC AVA $8 \mathrm{GHz}$ XY-plane . . . . . . . . . . . . 68

4.17 Simulated LTCC AVA $8 \mathrm{GHz}$ XZ-plane . . . . . . . . . . . . 68

4.18 Simulated near field probe result for LTCC AVA . . . . . . . . . . . . . 69

4.19 Cross-section of AVA and B-AVA . . . . . . . . . . 70

4.20 B-AVA schematic . . . . . . . . . . . . . . 71

4.21 Simulated B-AVA return loss . . . . . . . . . . . . . . . 72

4.22 Simulated LTCC B-AVA $6 \mathrm{GHz}$ XY-plane . . . . . . . . . . . . . 73

4.23 Simulated LTCC B-AVA $6 \mathrm{GHz}$ XZ-plane . . . . . . . . . . . . . 73

4.24 Simulated LTCC B-AVA $8 \mathrm{GHz}$ XY-plane . . . . . . . . . . . . . . . 73

4.25 Simulated LTCC B-AVA $8 \mathrm{GHz}$ XZ-plane . . . . . . . . . . . . 73

4.26 Bow-tie antenna . . . . . . . . . . . . . . . . 75

4.27 Triangular sheet antenna . . . . . . . . . . . . . . 75

4.28 PGP-TM $($ angled view $) \ldots \ldots \ldots \ldots \ldots$

4.29 PGP-TM topology . . . . . . . . . . . . . . . . 78

4.30 Simulated FR-4 PGP-TM return loss . . . . . . . . . . . . . 80

4.31 Simulated FR-4 PGP-TM 4 GHz XY-plane . . . . . . . . . . . . . . . 81

4.32 Simulated FR-4 PGP-TM 4 GHz YZ-plane . . . . . . . . . . . . 81

4.33 Simulated FR-4 PGP-TM 6 GHz XY-plane . . . . . . . . . . . . . 81 
4.34 Simulated FR-4 PGP-TM $6 \mathrm{GHz}$ YZ-plane . . . . . . . . . . . . 81

4.35 Simulated near field probe result for FR-4 PGP-TM . . . . . . . . . . 82

4.36 Simulated effect of parasitic element separation . . . . . . . . . . 84

4.37 Simulated LTCC PGP-TM return loss . . . . . . . . . . . . . . . . . 84

4.38 Simulated PGP-TM 3D radiation pattern at $6 \mathrm{GHz} \ldots \ldots$

4.39 Simulated LTCC PGP-TM 4 GHz XY-plane . . . . . . . . . . . . . 86

4.40 Simulated LTCC PGP-TM 4 GHz YZ-plane . . . . . . . . . . . . . 86

4.41 Simulated LTCC PGP-TM 6 GHz XY-plane . . . . . . . . . . . . 86

4.42 Simulated LTCC PGP-TM $6 \mathrm{GHz}$ YZ-plane . . . . . . . . . . . . . . 86

4.43 Simulated near field probe result for LTCC PGP-TM . . . . . . . . 87

4.44 OGP-TM schematic . . . . . . . . . . . . . . . 89

4.45 OGP-TM Return Loss . . . . . . . . . . . . . . . . . . 90

4.46 OGP-TM $4.7 \mathrm{GHz}$ XY-plane . . . . . . . . . . . . . . . . . 91

4.47 OGP-TM $4.7 \mathrm{GHz}$ XZ-plane . . . . . . . . . . . . . . . . . 91

4.48 PGP-TM with module . . . . . . . . . . . . . . . . . . . . 92

4.49 AVA with module . . . . . . . . . . . . . . . . . . 92

4.50 Circuit module $($ top $) \ldots \ldots \ldots$. . . . . . . . . . . . . 92

4.51 Circuit module (angled) . . . . . . . . . . . . . . . . . 92

5.1 Anechoic chamber . . . . . . . . . . . . . . . 96

5.2 Measured time domain pulse and spectral content . . . . . . . . . . . . 97

5.3 Measured $\left|S_{11}\right|$ for narrowband patch antenna . . . . . . . . . . . 98

5.4 Measured response in the time domain for the narrowband patch antenna . 99 xvi 


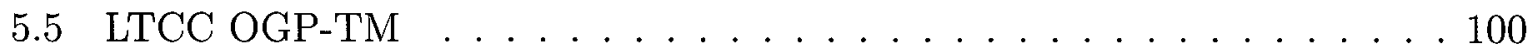

5.6 OGP-TM $\left|S_{11}\right|$ measurement . . . . . . . . . . . . . 100

5.7 Measured azimuthal patterns for OGP-TM at $4.7 \mathrm{GHz} \ldots \ldots 101$

5.8 Measured elevation patterns for OGP-TM at $4.7 \mathrm{GHz} \ldots \ldots$. . . . . . 101

5.9 Measured OGP-TM time domain response . . . . . . . . . . . . 102

5.10 FR-4 based AVA . . . . . . . . . . . . . . . . 102

5.11 Measured $\left|S_{11}\right|$ for FR-4 based AVA . . . . . . . . . . . 103

5.12 Measured $S_{21}$ of FR-4 based AVA . . . . . . . . . . . 103

5.13 Measured azimuthal patterns for FR-4 AVA at $4 \mathrm{GHz} \ldots \ldots . \ldots 104$

5.14 Measured elevation patterns for FR-4 AVA at $4 \mathrm{GHz} \ldots \ldots$. . . . . . 104

5.15 Measured azimuthal patterns for FR-4 AVA at $8 \mathrm{GHz} \ldots \ldots 105$

5.16 Measured elevation patterns for FR-4 AVA at $8 \mathrm{GHz} \ldots \ldots . . \ldots 5$

5.17 Measured FR-4 based AVA time domain response . . . . . . . . . . . 106

5.18 FR-4 based PGP-TM . . . . . . . . . . . . . . . 106

5.19 Measured $\left|S_{11}\right|$ for FR-4 based PGP-TM . . . . . . . . . . . 107

5.20 Measured $S_{21}$ of FR-4 based PGP-TM . . . . . . . . 108

5.21 Measured azimuthal patterns for FR-4 PGP-TM at $4 \mathrm{GHz} \ldots \ldots$. . . . . 109

5.22 Measured elevation patterns for FR-4 PGP-TM at $4 \mathrm{GHz}$. . . . . . . . 109

5.23 Measured azimuthal patterns for FR-4 PGP-TM at $6 \mathrm{GHz}$. . . . . . . . . 109

5.24 Measured elevation patterns for FR-4 PGP-TM at $6 \mathrm{GHz} \ldots \ldots$. . . . . 109

5.25 Measured FR-4 based PGP-TM time domain response . . . . . . . . . . 110

5.26 LTCC-based AVA . . . . . . . . . . . . . . . . . 111

xvii 
5.27 Measured $\left|S_{11}\right|$ of LTCC-based . . . . . . . . . . . . . . 111

5.28 Measured $S_{21}$ of LTCC-based AVA . . . . . . . . . . . 112

5.29 Measured azimuthal patterns for LTCC AVA at $6 \mathrm{GHz} \ldots \ldots$. . . . . . 113

5.30 Measured elevation patterns for LTCC AVA at $6 \mathrm{GHz} \ldots \ldots . \ldots$

5.31 Measured azimuthal patterns for LTCC AVA at $8 \mathrm{GHz} \ldots \ldots$. . . . . 113

5.32 Measured elevation patterns for LTCC AVA at $8 \mathrm{GHz} \ldots \ldots$. . . . . . 113

5.33 Measured LTCC-based AVA time domain response . . . . . . . . . . 114

5.34 LTCC-based PGP-TM . . . . . . . . . . . . . . . 115

5.35 Measured $\left|S_{11}\right|$ for LTCC-based PGP-TM . . . . . . . . . 116

5.36 Measured $S_{21}$ for LTCC-based PGP-TM . . . . . . . . . 116

5.37 Measured azimuthal patterns for LTCC PGP-TM at $4 \mathrm{GHz}$. . . . . . . 117

5.38 Measured elevation patterns for LTCC PGP-TM at $4 \mathrm{GHz} \ldots \ldots 17$

5.39 Measured azimuthal patterns for LTCC PGP-TM at $6 \mathrm{GHz} \ldots \ldots . . . .118$

5.40 Measured elevation patterns for LTCC PGP-TM at $6 \mathrm{GHz} \ldots \ldots$

5.41 Measured LTCC-based PGP-TM time domain response . . . . . . . . . 118

5.42 System $\left|S_{21}\right|$ trend with frequency . . . . . . . . . . . . . 122

xviii 


\section{Chapter 1}

\section{Introduction}

\subsection{Motivation}

The potential of UWB is enormous. UWB has the capability to provide extremely fast data rates at short transmission distances, requiring low power dissipation. These aspects could allow UWB the flexibility to displace popular wireless technology in use today, including Bluetooth and wireless LANs (WLANs-e.g., 802.11b, 802.11a, 802.11g).

Data communications are based upon the relationship discovered by Claude Shannon and Robert Hartley of Bell Labs. Consider Shannon's fundamental theorem for channel capacity:

$$
C=B \log \left(1+\frac{S}{N}\right) \quad[b / s]
$$

where $\mathrm{C}=$ maximum channel capacity, $\mathrm{B}=$ channel bandwidth, $\mathrm{S}=$ signal power and $\mathrm{N}=$ noise power. In conventional data communications and wireless applications, the frequencies of operation and bandwidth are fixed and strictly regulated. So, higher speeds can only be achieved with higher signal power. At large $\mathrm{S} / \mathrm{N}$ ratios, data rate scales as the logarithm of the power level. However, note that data rate scales linearly with bandwidth. This gives a UWB system the highest data-rate capability of all wireless 
techniques because no other communications system uses such a wide bandwidth.

Intense research is being conducted to develop UWB LNAs [1] [2], mixers [3] and entire front-ends [4]. However, a comparatively smaller amount of work is being done to develop UWB antennas. Designers are seemingly unaware of the tradeoffs between antenna design and transceiver complexity. In general, transceiver complexity increases with the introduction of new and more advanced wireless transmission techniques. Ultrawideband is no exception. Furthermore, it is becoming more costly, from a research and financial point of view, to extract maximum performance from a transceiver architecture. However, the antenna is an integral part of the transceiver that has thus far been relatively neglected. Rather than investing even more into the design of transceiver electronics, these resources can be more effectively spent on advanced antenna designs that increase the performance and decrease the complexity of the overall transceiver.

Nevertheless, the issue of antenna design in UWB systems is a major challenge [5]. Unlike conventional systems, the time domain behavior of the antenna is critical because of the pulse-type excitation used. New design guidelines are also needed to differentiate between narrowband and UWB antenna requirements.

Some studies on UWB antenna design have been published by Lamensdorf [6] and Hui $\mathrm{Wu}$ [7]. The primary design challenge is achieving a $7.5 \mathrm{GHz}$ impedance bandwidth from 3.1 $\mathrm{GHz}$ to $10.6 \mathrm{GHz}$. A return loss of greater than $10 \mathrm{~dB}$ is important in obtaining a high radiation efficiency. A high radiation efficiency is sought since UWB transmission is very low power and has a high sensitivity. Conductor and dielectric losses must be minimized to ensure maximum transmission range and overall functionality of the communication 
system. Pulse distortion is minimized when a two antenna system has a linear phase response and flat gain with frequency. The former ensures that each frequency component experiences the same delay when it is transmitted, while the later ensures that each frequency component is transmitted equally.

Ideally, the UWB antenna should be compact, planar, low cost and reliable. Compatibility and ease of integration with electronics for mobile communications is also desirable. Moreover, parasitic effects associated with the assembly of transceiver electronics and packaging elements are compounded in UWB systems. LTCC is a promising packaging technology that has the potential to satisfy these needs, while minimizing parasitic effects. Because of these challenges and the relative newness of UWB, it is significant to explore novel UWB antenna designs in the context of LTCC integration.

\subsection{Thesis Goals}

The primary goal of this thesis is to design and fully characterize several UWB compliant antennas that are planar and have circuit integration possibilities. To this end, the need for practical design guidelines for UWB antennas is addressed first. The second objective is the development of an in-house electromagnetic simulator based on the FDTD technique. This simulator provides maximum flexibility in the areas of feed mechanisms, source waveform selection and sampling locations. HFSS, a well known commercially available tool, is used to validate the results obtained by the FDTD technique and to aid in the design of UWB antennas. Based on the newly formulated guidelines, promising antenna topologies are selected before an investigation into several fundamental parameters of 
UWB antenna operation is conducted. The effects of geometry, substrate permittivity, size, parasitic elements and different ground plane configurations on antenna performance in the frequency and time domain is studied.

The candidate UWB antennas include i) a triangular monopole with an orthogonal ground plane ii) a triangular monopole with a partial ground plane $i i i)$ and an antipodal Vivaldi antenna. These antenna types are inherently broadband in nature and do not require a broadband balun for matching. Additionally, the usefulness of an LTCC substrate for combined antenna and electronic circuit applications is investigated. The final objective is to validate the formulated guidelines for a UWB link along with the design and analysis of the antennas by comparing the simulated results with the measured values.

\subsection{Thesis Outline}

The two primary topics discussed in this thesis are $i$ ) UWB antenna design and ii) FDTD simulation.

The first part of Chapter 2 provides some background information on UWB radio including the history, theory and future outlook for the technology. Implementation schemes and regulations will also be presented. The next section will give a summary of important parameters needed for the study of narrowband, broadband or UWB antennas including directivity, gain, efficiency, radiation patterns and others. LTCC as a attractive packaging technology is described and a critical overview of current UWB antenna requirements is given before several guidelines are formulated. Chapter 2 concludes with an introduction to UWB principles and a detailed overview of common broadband antennas. 
A detailed theoretical overview of the FDTD technique is given in Chapter 3, including a summary of the advantages of this simulation technique with regards to antenna modeling. An introduction to Maxwell's equations and Yee's FDTD formulation are the first topics covered. Important analysis considerations such as numerical dispersion and stability are defined. Excitation methods and absorbing boundary conditions are also discussed. The chapter concludes with an overview of the FDTD program structure and the results generated from the simulation of a simple patch antenna are presented and compared to measured published results.

Five different antenna designs are described in Chapter 4. The design, simulation and testing of these antennas was performed at Carleton University and CRC. A non-planar triangular monopole along with LTCC and FR-4 implementations of a planar triangular monopole and compact antipodal Vivaldi antenna are studied. UWB antenna design is an iterative process involving a large number of inter-dependent design parameters; including geometry, permittivity, size and overall topology. These parameters have an impact on impedance bandwidth, radiation characteristics, efficiency and pulse response. Design procedures for each antenna are outlined and a discussion of the simulation results is given.

Measured results, including frequency domain s-parameters and time domain pulse responses are presented in Chapter 5 . The simulation results contained in Chapter 4 are compared to the measured performance and a discussion on their agreement is included. A comparison between antennas based on LTCC and FR-4 is made to identify the relative performance and feasibility of fabricating antennas on an LTCC substrate. The conclusion 
in Chapter 6 concentrates on UWB antenna design and emphasizes the encouraging results shown in this thesis. Since UWB is relatively new, several suggestions for future work are made at the end of this chapter.

The bibliography can be found at the end of the thesis. 


\section{Chapter 2}

\section{Technology Overview}

This chapter provides a background on UWB technology that was briefly introduced in Chapter 1. The two modern implementations of UWB are very different and have their own advantages and disadvantages. Ultra-wideband design differs from traditional narrowband design in many aspects. However, the fundamental antenna parameters (with minor alterations) are still applicable. Successful UWB design hinges on the fulfilment of several uncommon requirements for antennas. System aspects of UWB and other important principles will also be discussed.

\subsection{About UWB}

UWB is an emerging radio design standard that promises to deliver very high transmission rates while using little power and being low cost. The allocated frequency band of $3.1 \mathrm{GHz}$ to $10.6 \mathrm{GHz}$ is already heavily populated by other radio standards but UWB uses such low power signals that a UWB transmission will appear as noise to those radios. Transmission ranges are limited to 10 or a few 10s of meters. This limits UWB to be primarily an indoor wireless technology. 
UWB is being targeted as a cable replacement technology intended to support high bandwidth transmissions such as high definition television or digital audio. No other current radio standard has this capability. Figure 2.1 depicts some of the commercial applications for UWB.

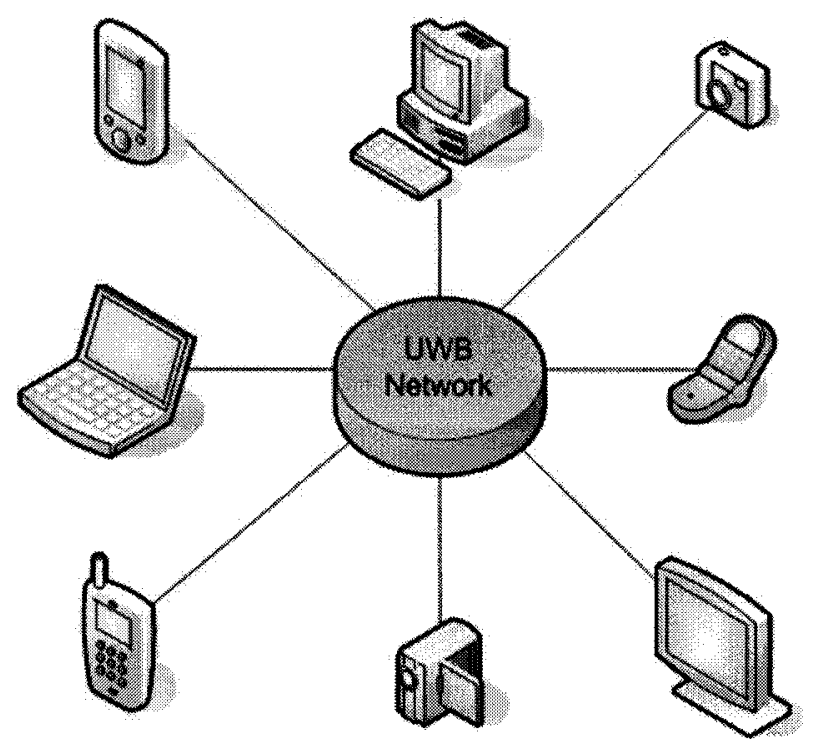

Figure 2.1: UWB Network

UWB can simplify home theater setup by eliminating the need to use wires to connect all the devices such as flat panel televisions and DVD players. UWB can remove most of the clutter around personal computers (PC) by making a wireless connection between PCs and flat panel monitors or other peripheral devices such as scanners and video cameras.

\subsection{History of UWB}

The history of UWB dates back to the era of baseband or impulse radios. In 1893, Heinrich Hertz used a spark discharge to generate electromagnetic waves. However, this type of radio was short lived and was superseded by narrowband systems in the early 
1900's [8]. Research in the UWB field continued and several patents were filed on impulse radio systems during $1942-1945$.

The 1960's saw two very important advancements in UWB technology. First, the origin of UWB technology can be traced back to the work of Gerald F. Ross who developed a time domain method to fully describe the transient behavior of microwave networks through their characteristic impulse response $h(t)$ [9]. Later, the output of such a system to any arbitrary input $x(t)$ could be determined by the convolution integral:

$$
y(t)=\int_{-\infty}^{\infty} h(u) x(t-u) d u
$$

The advent of the sampling oscilloscope by Hewlett-Packard in 1962 represented the second important technological breakthrough for UWB. This equipment was necessarily for the observation and measurement of the impulse response of microwave networks [9].

The term UWB was first used by the U.S. Department of Defense in 1989. Before then, the technology was known under several names such as baseband, carrier-free or impulse radio. The advancement of technology in the 1990's allowed for the commercialization of UWB technology. An updated definition for UWB was given by the FCC in 2005 . For indoor applications, the FCC defines UWB as any signal that occupies more than $500 \mathrm{MHz}$ bandwidth in the $3.1 \mathrm{GHz}$ to $10.6 \mathrm{GHz}$ band and that meets the spectrum mask shown in Figure 2.2 [10].

\subsection{Two flavors of UWB}

At the present time an official physical layer (PHY) standard for indoor UWB has not been finalized. The IEEE 802.15.3a task group is working to unify two PHY proposals 


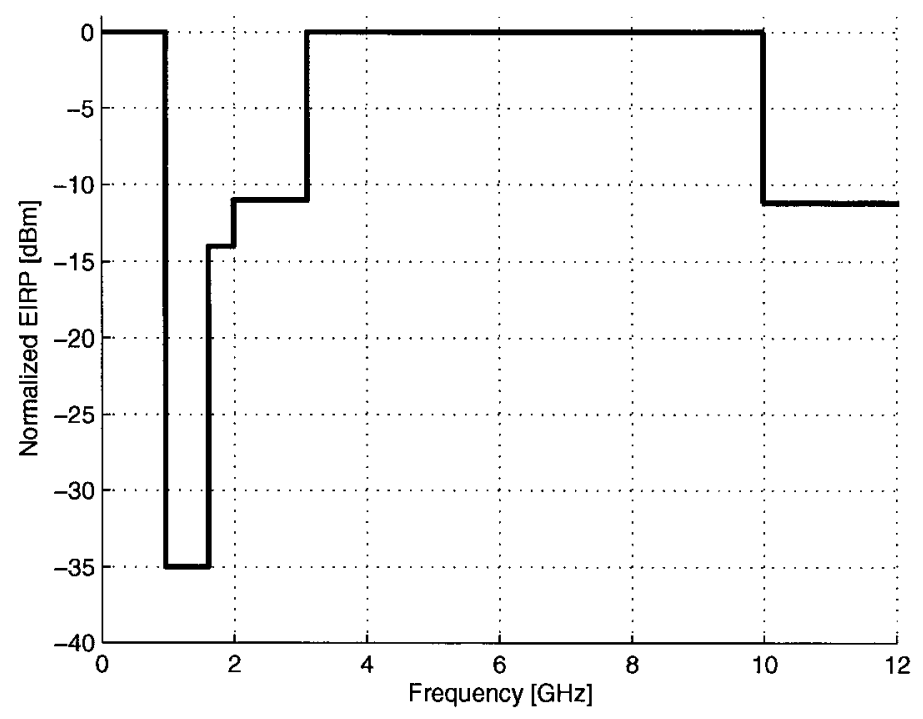

Figure 2.2: FCC Indoor Spectrum Mask

for UWB put forth by two organizations composed of leading companies. The first group, promoted by Intel, Hewlett Packard, Panasonic and others, is proposing a multi-band approach based on orthogonal frequency division multiplexing (OFDM). The division of the $7.5 \mathrm{GHz}$ bandwidth by the multi-band OFDM alliance (MBOA) is shown here:

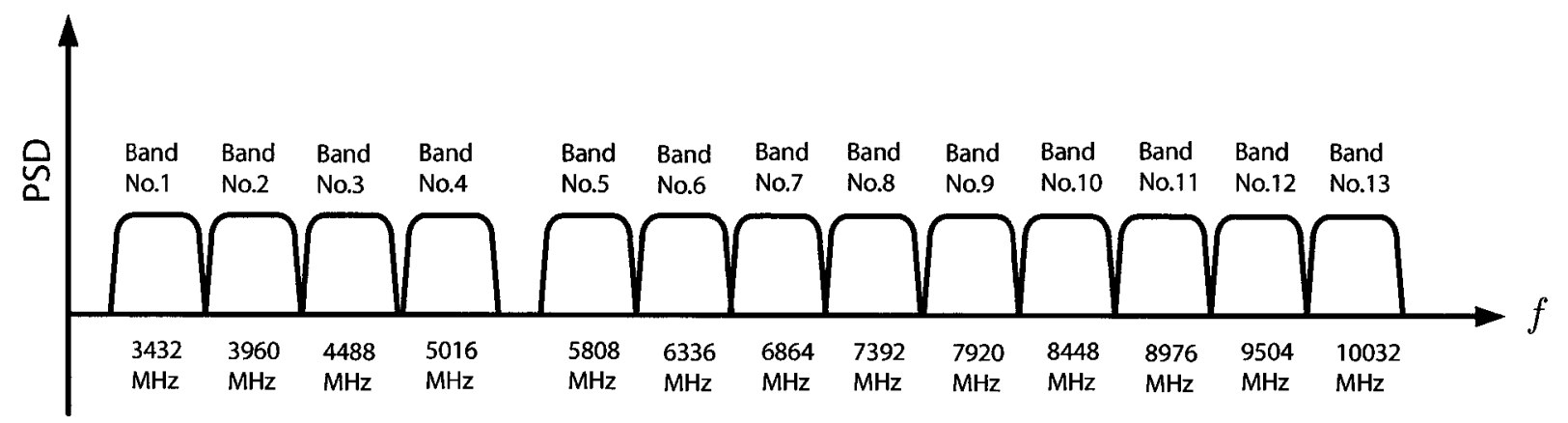

Figure 2.3: OFDM Bands

The system uses a total of 122 sub-carriers that are modulated using quadrature phase shift keying (QPSK). The system also uses a time-frequency code (TFC) to interleave coded data over the frequency bands. Initially, this scheme will support a maximum 
transmission rate of $480 \mathrm{Mb} / \mathrm{s}$ at a distance of $9.3 \mathrm{~m}$. The main advantage of this system is that it utilizes a proven architecture based on OFDM. Disadvantages include an increase in the complexity of the transceivers due to the parallel processing of the sub-bands.

The second group, promoted by Motorola, Synopsys and others, is proposing a direct sequence UWB (DS-UWB) model that supports two independent bands of operation [11]. The lower band occupies the spectrum from $3.1 \mathrm{GHz}$ to $4.85 \mathrm{GHz}$ and the upper band occupies the spectrum from $6.2 \mathrm{GHz}$ to $9.7 \mathrm{GHz}$. The division of the $7.5 \mathrm{GHz}$ bandwidth by DS-UWB is shown below:

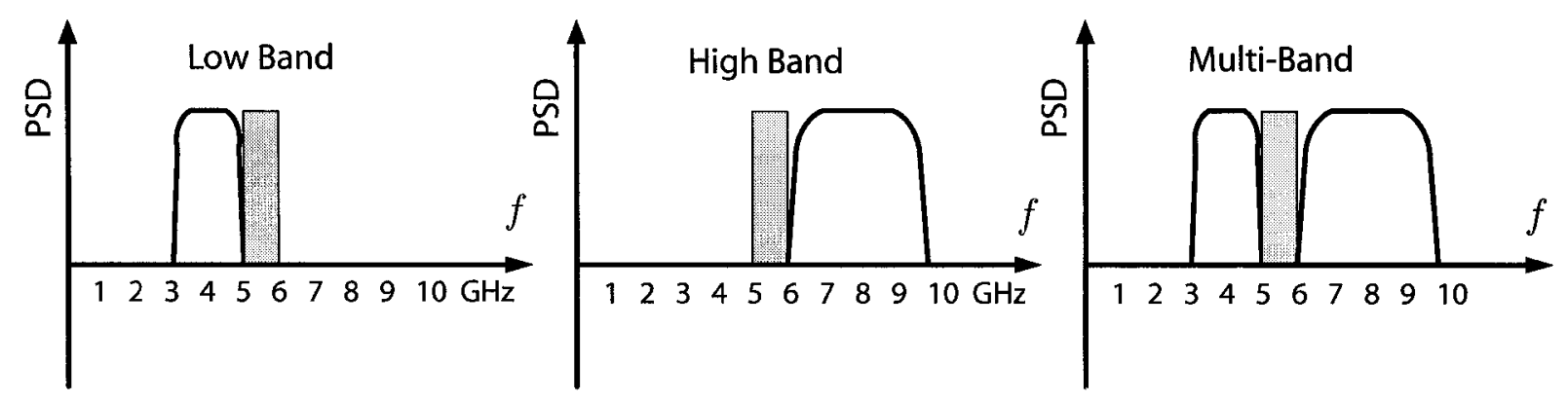

Figure 2.4: DS Bands

The proposed UWB system employs direct sequence spreading of binary phase shift keying (BPSK) and quaternary bi-orthogonal keying (4BOK) UWB pulses to modulate the data symbols. Prototype devices utilizing the lower band have demonstrated transmission rates up to $480 \mathrm{Mb} / \mathrm{s}$. The main advantage of this proposal is its significantly reduced digital baseband complexity. However, the DS-UWB architecture is entirely new and the extra development time necessary may make this scheme more expensive.

Both of these methods of utilizing the UWB spectrum are highly developed and promise to make personal devices with high speed wireless connectivity $(>480 \mathrm{Mb} / \mathrm{s})$ 
a reality. However, the DS-UWB implementation is being pursued in this thesis because it presents the greatest challenge to antenna designers. Furthermore, an antenna designed to meet the DS-UWB specifications will automatically satisfy those of the MBOA.

\subsection{Important Antenna Parameters}

The official IEEE definition of an antenna is "That part of a transmitting or receiving system that is designed to radiate or receive electromagnetic waves". More simply, an antenna acts as a transformer to convert guided waves on a transmission structure into free space waves. Important antenna performance parameters such as, radiation pattern, directivity, gain, polarization, impedance, transient response, bandwidth and efficiency are used to characterize an antenna's suitability for a certain application. The following concepts will be important in the study of the antennas presented in Chapter 4.

\subsubsection{Radiation Pattern}

The radiation pattern is a graphical representation of the electric and magnetic field strengths surrounding an antenna at a particular position in space. This space can be divided into three regions: i) reactive near field ii) radiating near field and iii) the far field. The far field is where most antenna measurements are taken and is given by:

$$
R_{f f}=\frac{2 D^{2}}{\lambda}
$$

Where D is the largest physical dimension of the antenna. For electrically small antennas, Equation 2.2 may give a far-field distance that is too small; in this case a minimum value of $R_{f f}=2 \lambda$ should be used [12]. The field strength is quantified with the Poynting 
vector expression of Equation 2.3, which gives the complex power density surrounding the antenna.

$$
\mathbf{S}=\frac{1}{2}\left(\mathbf{E} \times \mathbf{H}^{*}\right)
$$

A spherical co-ordinate system is used to define radiation patterns. The $\mathrm{x}-\mathrm{y}$ plane contains the phi-component where the theta-component is 90 degrees $\left(\phi\right.$ where $\theta=90^{\circ}$ ) and is usually referred to as the azimuthal plane. The $\mathrm{x}-\mathrm{z}$ plane contains the theta-component where phi is zero degrees and usually indicates the elevation plane $\left(\theta\right.$ where $\left.\phi=0^{0}\right)$. A pattern taken in a plane that contains the electric field vector is called the the Eplane pattern. Similarly, a pattern taken in the plane that contains the magnetic field vector is called the H-plane pattern. The E and H-plane patterns, in general, are called the principal plane patterns. The antennas designed in this thesis display two different patterns: i) omni-directional and ii) directional. The radiation pattern for an ideal dipole is doughnut shaped. A dipole has an omni-directional pattern, which is non directional in the azimuthal plane and directional in the elevation plane. A wave-guide horn is an example of a directional antenna, which receives energy more effectively in one direction than the other.

\section{Radiation Pattern Lobes}

For a directional antenna the radiation pattern will consist of many lobes or radiation peaks. The largest lobe is called the main lobe and is the region in space with the greatest field intensity. The lobes located to either side of the main lobe are called minor lobes, while the lobe directly behind is called the back lobe. Table 2.1 displays the most 
important parameters related to radiation patterns.

Table 2.1: Radiation Pattern Properties

\begin{tabular}{|l|l|}
\hline Parameter & Description \\
\hline \hline Half-power beam width (HPBW) & $\begin{array}{l}\text { Is the angle between the two direc- } \\
\text { tions in which the radiation inten- } \\
\text { sity is one-half the maximum value } \\
\text { of the beam }\end{array}$ \\
\hline $\begin{array}{l}\text { Beam width between first nulls } \\
\text { (BWFN) }\end{array}$ & $\begin{array}{l}\text { Is the angle between the first nulls } \\
\text { on either side of the main beam }\end{array}$ \\
\hline Front to back ratio (FBR) & $\begin{array}{l}\text { Is the ratio of the power of the main } \\
\text { lobe to the power of the back lobe }\end{array}$ \\
\hline
\end{tabular}

\subsubsection{Bandwidth}

The bandwidth of an antenna refers to the frequency range over which the performance of the antenna is acceptable. Impedance bandwidth and pattern bandwidth are the two main factors.

\section{Impedance Bandwidth}

Impedance bandwidth specifies a range of frequencies for which the antenna accepts more than $90 \%$ of the power applied to its input terminals. This is usually represented by a return loss (RL) of $10 \mathrm{~dB}$ or greater or a voltage standing wave ratio (VSWR) of at most 2.0. In general, the input impedance of the antenna is composed of a real and imaginary part:

$$
Z_{A}=R_{A}+j X_{A}
$$

The first term is the input resistance and represents dissipation, which can be due to radiation or ohmic losses. Except for electrically small antennas, radiation dominates. 
The second term is the input reactance and represents the power stored by the antenna in the near field. Equation 2.4 is valid for both a receiving and transmitting antenna due to reciprocity. The amplitude of the reflected voltage wave normalized to the amplitude of the incident voltage wave is called $\Gamma$ and is given by Equation 2.5. Transmission line theory can be used to obtain $\Gamma$.

$$
\Gamma=\frac{V_{0}^{-}}{V_{0}^{+}}=\frac{Z_{L}-Z_{0}}{Z_{L}+Z_{0}}
$$

The return loss and the VSWR are dependent on $\Gamma$ and can be derived from Equations 2.6 and 2.7 , representing the voltage and current waves on a line, respectively.

$$
\begin{aligned}
& V(z)=V_{0}^{+}\left[e^{-j \beta z}+\Gamma e^{j \beta z}\right] \\
& I(z)=\frac{V_{0}^{+}}{Z_{0}}\left[e^{-j \beta z}-\Gamma e^{j \beta z}\right]
\end{aligned}
$$

The time-average power flow along the line, given in Equation 2.8, shows that the average power flow is a constant along the line and is equal to the incident power minus the reflected power.

$$
P_{a v}=\frac{1}{2} \operatorname{Re}\left[V(z) I(z)^{*}\right]=\frac{1}{2} \frac{\left|V_{0}^{+}\right|^{2}}{Z_{0}}\left(1-|\Gamma|^{2}\right)
$$

Return loss represents the lost or reflected power due to a load that is mismatched and can be calculated using:

$$
R L=-20 \log |\Gamma| \quad[d B]
$$

The return loss cannot be obtained directly by the FDTD simulator since it is based in the time domain. The Fourier transform must be performed on the time domain incident and reflected voltage waves to obtain $\Gamma$, then the return loss can be calculated with Equation 2.9. A more detailed discussion is provided in Chapter 3. 


\subsubsection{Directivity}

Directivity is a measure of the focusing ability of an antenna and is defined as the ratio of the maximum radiation intensity (power per unit solid angle) in the main beam to the average power over all space (average over a sphere). Equation 2.10 shows that a fictitious isotropic radiator has a radiation intensity independent of angles $\theta$ and $\phi$, and equal to the radiated power averaged over the entire solid angle.

$$
U_{0}=\frac{P_{r a d}}{4 \pi}
$$

All antennas have a directivity greater than one defined by:

$$
D(\theta, \phi)=\frac{U(\theta, \phi)}{U_{0}}=\frac{4 \pi U(\theta, \phi)}{P_{r a d}}
$$

\subsubsection{Efficiency}

An antenna can not radiate $100 \%$ of the power delivered to its input terminals because of conductive, dielectric and other losses. Equation 2.12 defines the radiation efficiency as being a product of the conduction efficiency and dielectric efficiency.

$$
e_{r a d}=e_{c} e_{d}
$$

However, the above equation is valid at only a single frequency. This is sufficient for narrowband systems but not for UWB systems where the efficiency can be a function of frequency. Equation 2.13 shows that the radiation efficiency is a function of frequency related to the dielectric loss since, in general, the dielectric loss dominates over the conductor loss.

$$
e_{r a d}(f)=e_{c} e_{d}(f)
$$


The overall antenna efficiency includes losses due to an impedance mismatch at the input port of the antenna. If the antenna has a reflection coefficient of $\Gamma$, then the antenna efficiency at a single frequency is given by [13]:

$$
e_{a}=\left(1-|\Gamma|^{2}\right) e_{r a d}
$$

\subsubsection{Gain}

The gain of an antenna is a parameter closely related to directivity and efficiency. Gain is the directivity reduced by the losses on the antenna and is given by [14]:

$$
G(\theta, \phi)=e_{r} D=\frac{4 \pi U(\theta, \phi)}{P_{i n}}
$$

This definition does not include mismatch or impedance losses and when direction is not given, maximum gain is assumed.

\subsubsection{Polarization}

The polarization of a wave refers to the temporal orientation and magnitude of its electric field. The magnetic field behaves similarly in a plane perpendicular to that of the electric field. The instantaneous electric field vector is given by:

$$
E_{t o t}=E_{x} \hat{\mathbf{x}}+E_{y} \hat{\mathbf{y}}=E_{1} \cos (\omega t) \hat{\mathbf{x}}+E_{2} \cos (\omega t+\delta) \hat{\mathbf{y}}
$$

Where, $\delta$ is the phase by which the $\mathrm{y}$-component leads the $\mathrm{x}$-component. Polarization can be either linear, circular or elliptical with the latter being the general case. The antennas in this thesis are all linearly polarized. Table 2.2 shows the two mutually orthogonal components for these antennas that can exist. The polarization of an antenna is 
Table 2.2: Polarization States

\begin{tabular}{|c|c|}
\hline Polarization & Description \\
\hline \hline Vertical Linear & $\delta=0^{\circ}$ and $E_{1}=0$ \\
\hline Horizontal Linear & $\delta=0^{0}$ and $E_{2}=0$ \\
\hline
\end{tabular}

defined as the polarization of the wave radiated by the antenna when transmitting [14]. Typically, antennas will exhibit some form of elliptical polarization. Usually, the predominant component is the desired one and is known as the co-polarized component. The minor component is undesired and referred to as the cross-polarized component. A cross-polarization level of $20 \mathrm{~dB}$ below the co-polarized value is preferred. As shown by Equation 2.16, polarization is defined at a single frequency but for a UWB application maintaining a stable polarization across a wide frequency bandwidth can be challenging. Chapters 4 and 5 will show how the polarization of an antenna can change significantly with frequency. A more detailed discussion on antenna polarization can be referenced in $[13]$ and [14].

\subsection{Low Temperature Co-fired Ceramic RF Modules}

LTCC RF modules are an excellent packaging option for microwave circuits. They offer a high performance method to integrate active and passive components in a most compact way. Figure 2.5 shows the 3-D structure of a typical LTCC module. Current LTCC modules can contain up to 25 layers and have a maximum size of $150 \mathrm{~mm}^{2}$. Each layer is made from a ceramic green sheet, which then undergoes a specialized process flow to create the final RF module. The LTCC process uses thin unfired glass/ceramic tape sheet 


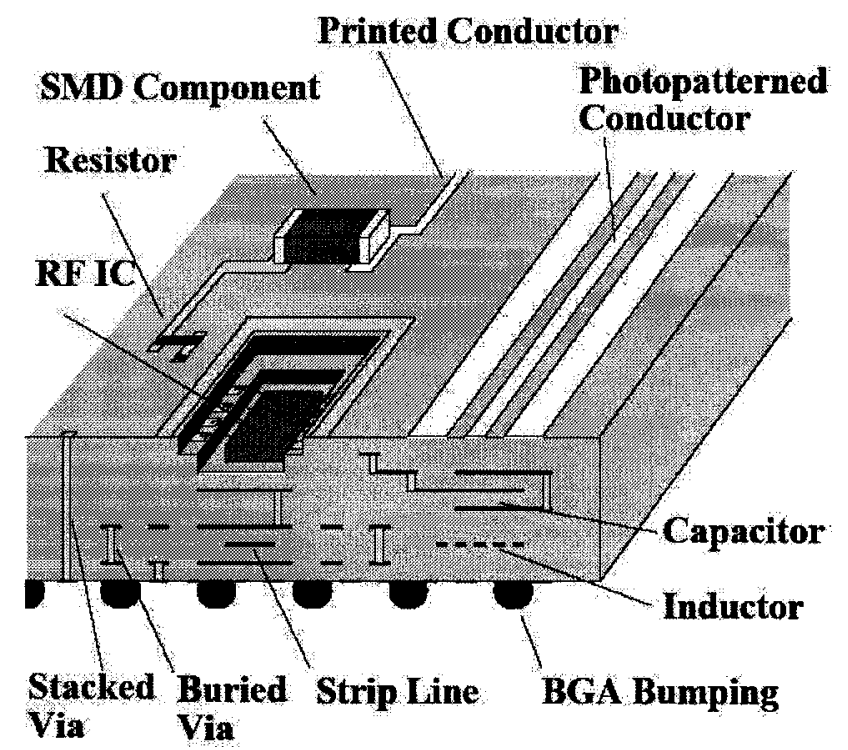

Figure 2.5: 3-D LTCC Structure

layers, on which the conductors are made using conventional thick film printing. For large metal layers, such as those necessary to pattern the antennas designed in this thesis, a special method is employed to avoid substrate warping. This method involves creating a mesh or grid on the area of the antenna, which is then filled with a metal paste to form the metal layer.

Interconnections through a single tape layer are made by vias, which are typically punched holes filled with conductive paste. Separate tape layers are stacked, laminated and finally co-fired to form a monolithic multilayered substrate consisting of even tens of layers. Material systems, having different electrical or mechanical properties are available from several manufacturers [15]. Many different LTCC tape materials can be selected based on design requirements, from general purpose low cost tape such as DuPont951 to very low-loss FerroA6-S tape that has the capability to use embedded resistors. Table 2.3 contains some of the available tape materials and their properties. 
Table 2.3: LTCC Tape Material Data

\begin{tabular}{|l|c|c|c|c|}
\hline & DuPont 951 & FerroA6-S & DuPont 943 & Heraeus CT 2000 \\
\hline \hline $\begin{array}{l}\text { Dielectric } \\
\text { constant }\end{array}$ & 7.8 & 5.9 & 7.5 & 9.1 \\
\hline $\begin{array}{l}\text { Dissipation } \\
\text { Factor }\end{array}$ & $0.15 \%$ & $<0.2 \%$ & $<0.1 \%$ & $0.1 \%$ \\
\hline $\begin{array}{l}\text { Breakdown } \\
\text { Voltage }\end{array}$ & $>1000 \mathrm{~V} / 25 \mathrm{~m}^{2}$ & $>15000 \mathrm{~V} / 93 \mathrm{~m}^{2}$ & $\mathrm{~N} / \mathrm{A}$ & $>1000 \mathrm{~V} / 25 \mathrm{~m}^{2}$ \\
\hline Color & $\mathrm{Blue}$ & $\mathrm{White}$ & $\mathrm{Blue}$ & $\mathrm{Blue}$ \\
\hline $\begin{array}{l}\text { Thermal Con- } \\
\text { ductivity }\end{array}$ & $3 \mathrm{~W} / \mathrm{mK}$ & $2 \mathrm{~W} / \mathrm{mk}$ & $3 \mathrm{~W} / \mathrm{mk}$ & $\mathrm{N} / \mathrm{A}$ \\
\hline $\begin{array}{l}\text { Thermal Ex- } \\
\text { pansion }\end{array}$ & $5.8 \mathrm{ppm} / \mathrm{K}$ & $7 \mathrm{ppm} / \mathrm{K}$ & $5.3 \mathrm{ppm} / \mathrm{K}$ & $8.5 \mathrm{ppm} / \mathrm{K}$ \\
\hline $\begin{array}{l}\text { Flexural } \\
\text { Strength }\end{array}$ & $320 \mathrm{MPa}$ & $>210 \mathrm{MPa}$ & $\mathrm{N} / \mathrm{A}$ & $240 \mathrm{MPa}$ \\
\hline $\begin{array}{l}\text { Shrinkage\%: } \\
\begin{array}{l}\text { Z-axis, XY- } \\
\text { axis }\end{array}\end{array}$ & $15,12.7$ & $27,15.5$ & 13,10 & $14,11.5$ \\
\hline
\end{tabular}

These tape materials are ideal for Microwave and RF applications because of their stable dielectric constants, low dielectric and line losses, excellent thermal matching to active devices at high operating temperatures and good environmental stability [15].

An important advantage of LTCC is the possibility to integrate passive and active components on different layers. This makes it possible to create complex but compact designs. Transmission lines, inductive elements, capacitive elements, resistive elements and even complex structures like resonators and filters can be buried between layers. Cavities can be created to contain RF integrated circuits making stand-alone transceiver modules, complete with antenna, possible [16] [17]. 


\subsection{A Critical Overview of Current UWB Antenna Requirements}

In narrowband systems, conventional antenna parameters described earlier such as impedance bandwidth, gain and radiation pattern are sufficient to fully describe the performance of an antenna. However, with UWB the situation is more complicated. Traditional antenna parameters are not adequate to completely describe a UWB antenna. When designing a UWB antenna, its time domain behavior must be understood. Simply choosing to design an antenna with multiple resonances to effectively create a broad bandwidth can lead to significant waveform dispersion [18]. For most antenna designers it is an unexpected requirement to evaluate an antenna in the time domain. Many UWB antenna designs have been presented that achieve a VSWR bandwidth of $500 \mathrm{MHz}$ or greater but neglect to evaluate the response in the time domain to a pulse excitation [19] [20] [21], potentially leading to significant waveform distortion [22]. A concurrent time domain and frequency domain design of such antennas is helpful. There is a need to define a list of parameters and guidelines that will help designers meet UWB antenna requirements. It is also important to determine which frequency domain parameters give a good indication of the time domain performance of a UWB antenna. The next section makes several observations.

\subsubsection{UWB Antenna Requirements}

All traditional narrowband antenna parameters apply to the design of UWB antennas. However, they can be be modified and new ones can be added to better suit UWB's 
unique challenges. Table 2.4 outlines these parameters.

Table 2.4: UWB Antenna Requirements

\begin{tabular}{|c|l|}
\hline VSWR Bandwidth & $\begin{array}{l}\text { Full band: } 3.1 \mathrm{GHz}-10.6 \mathrm{GHz} \text { or } \\
\text { Sub band: } 3.1 \mathrm{GHz}-4.85 \mathrm{GHz}, 6.2 \mathrm{GHz}- \\
9.7 \mathrm{GHz}\end{array}$ \\
\hline$S_{21}$ (two antenna system) & Flat with linear phase response \\
\hline Polarization & Linear (Horizontal or Vertical) \\
\hline Efficiency & High (>75\%) \\
\hline Radiation Pattern & $\begin{array}{l}\text { Base antenna: Omni-directional } \\
\text { Mobile antenna: Omni or directional }\end{array}$ \\
\hline Directivity and Gain & $\begin{array}{l}\text { Base antenna: Low } \\
\text { Mobile antenna: High }\end{array}$ \\
\hline Physical Topology & $\begin{array}{l}\text { Compact and planar without broadband } \\
\text { balun or matching network requirement }\end{array}$ \\
\hline Substrate & $\begin{array}{l}\text { Low loss with stable dielectric constant } \\
\text { and high cut-off frequency }\end{array}$ \\
\hline
\end{tabular}

A UWB antenna must have a full band VSWR bandwidth of $3.1 \mathrm{GHz}$ to $10.6 \mathrm{GHz}$ or sub band VSWR bandwidths of $3.1 \mathrm{GHz}$ to $4.85 \mathrm{GHz}$ or $6.2 \mathrm{GHz}$ to $9.7 \mathrm{GHz}$ (based on the DS-UWB implementation). Other factors important for a highly efficient antenna include low dielectric and conductor losses, which can be achieved by using ceramic substrates and gold or silver conductors. The transmission coefficient of a two antenna system, or $S_{21}$, is an important frequency domain indicator of the time domain performance of a UWB antenna [22]. The magnitude of $S_{21}$ should be flat over the same bandwidth that the VSWR is low. All of the frequency components in the frequency range where $S_{21}$ is near constant will be transmitted equally. Verifying that the phase of $S_{21}$ is linear over the bandwidth should not be omitted. A linear phase represents the application of a constant 
time delay to all the frequency components of the transmitted signal. Achieving these two aspects of the transmission coefficient helps to minimize the distortion and dispersion caused by a UWB antenna. Finally, a mobile device can be more power efficient when a higher gain directional antenna is used.

\subsubsection{Directivity and System Performance}

The overall system performance can be maximized by taking advantage of the directionality of some antennas. In general, a dipole antenna with an omni-directional radiation pattern will have a gain of about $2.2 \mathrm{dBi}$ (relative to an ideal isotropic radiator). A directional antenna, such as a horn antenna, will have a much higher gain because it can focus more energy in one direction.

It is desirable to maximize the power level at the receiver for a given amount of transmitted power. The Friis transmission equation (Equation 2.17) relates the amount of power received $\left(P_{R}\right)$ at the terminals of the receiving antenna, to the amount of power transmitted $\left(P_{T}\right)$ at the terminals of the transmitting antenna, where the two antennas are in the far-field of each other, separated by a distance R [13]. The last term in the Friis equation is called the polarization loss factor (PLF) and is used to quantify the amount of power lost due to polarization mismatch, where $\hat{\rho}_{T}$ and $\hat{\rho_{R}}$ define the transmit and receive polarization unit vectors, respectively.

$$
P_{R}=P_{T} G_{T} G_{R}\left(\frac{\lambda}{4 \pi R}\right)^{2}\left(1-\left|\Gamma_{T}\right|^{2}\right)\left(1-\left|\Gamma_{R}\right|^{2}\right)\left|\hat{\rho}_{T} \bullet \hat{\rho}_{R}\right|^{2}
$$

The choice of antennas has a direct impact on system performance. In particular, the requirement to maintain a flat system $S_{21}$ is dependent on the ability to achieve a 
constant $P_{T X}(f) G_{T X}(f)$ product that is close to the regulatory limit. However, path loss has a frequency dependance described as $1 / f^{2}$ and is given by:

$$
\text { path loss }=\left(\frac{c}{4 \pi R f}\right)^{2}
$$

In decibels, the free space path loss dependency from $1 \mathrm{GHz}$ to $10 \mathrm{GHz}$ is given by:

$$
20 \log \left(\frac{1}{f_{u}}\right)-20 \log \left(\frac{1}{f_{l}}\right)=20 \log \left(\frac{1}{10}\right)-20 \log \left(\frac{1}{1}\right)=-20 \mathrm{~dB} / \mathrm{dec}
$$

Antenna gain also has a frequency dependance and is defined in terms of antenna effective aperture $A_{e}$ as:

$$
G(f)=\frac{4 \pi A_{e}(f)}{\lambda^{2}}=\frac{4 \pi A_{e}(f) f^{2}}{c^{2}}
$$

Antenna gain and path loss are products in the Friis equation whose frequency dependencies cancel out to leave antenna effective aperture as the dominant term that effects link performance (assuming perfect impedance and polarization matching). The antenna effective aperture of a constant gain or omni-directional antenna decreases as $1 / f^{2}$. Conversely, the effective aperture of most directional antennas increases as $f^{2}$ causing the radiation pattern to narrow and for gain to increase.

Four distinct combinations of electrically small transmit and receive antennas are listed in Table 2.5 along with the relationship between gain and received power. Case 1 refers to a communications link consisting of omni-directional transmit and receive antennas; case 2 consists of an omni-directional transmitter and directional receiver; cases 3 and 4 both consist of directional transmit and receive antennas but have decreasing or flat transmit power spectrums, respectively. In case 1 , the constant gain antennas cause the 
received power to decrease as $1 / f^{2}$ (or $-20 \mathrm{~dB}$ per decade) because of the path loss. This shortcoming must be compensated for by the transmitter designer. Case 2 does not have this shortcoming since the increasing gain of the receive antenna cancels the increasing path loss with respect to frequency. This is also true in case 3, however a transmit power that decreases as $1 / f^{2}$ is used to achieve a flat $P_{R X}(f)$ spectrum. In case 4 a flat transmit power results in a $P_{R X}(f)$ spectrum that increases as $f^{2}$ (or $20 \mathrm{~dB}$ per decade). In cases 2 and 3 the received power will be higher relative to case 1 [18]. Omni-directional and directional antennas will be developed in Chapter 4 to verify the effect of directivity on system performance. Chapter 5 will present measurements of antenna systems that correspond to cases 1 and 4 .

Table 2.5: Gain and Link Performance for Electrically Small Antennas

\begin{tabular}{|c|c|c|c|c|}
\hline & Case 1 & Case 2 & Case 3 & Case 4 \\
\hline \hline$P_{T X}(f)$ Spectrum & Flat & Flat & $\downarrow 1 / f^{2}$ & Flat \\
\hline TX Ant. Gain & Flat & Flat & $\uparrow f^{2}$ & $\uparrow f^{2}$ \\
\hline RX Ant. Gain & Flat & $\uparrow f^{2}$ & $\uparrow f^{2}$ & $\uparrow f^{2}$ \\
\hline$P_{R X}(f)$ Spectrum & $\downarrow 1 / f^{2}$ & Flat & Flat & $\uparrow f^{2}$ \\
\hline
\end{tabular}

It may be helpful to base a UWB antenna design on a topology that is considered inherently broadband. The next section will present many different types of broadband antennas that have the potential to satisfy the requirements of Table 2.4 .

\subsection{UWB Principles}

This section provides a detailed overview of some common broadband antennas that are not suitable for UWB, while Chapter 4 will present practical candidates. An assessment of 
each antenna topology is made based on the following criteria: (i) impedance bandwidth of $3.1 \mathrm{GHz}$ to $4.85 \mathrm{GHz}$ or $6.2 \mathrm{GHz}$ to $9.7 \mathrm{GHz}$ (ii) good time domain performance (iii) linear polarization (iv) small size. Potential performance is based upon theoretical considerations and design feasibility. From this assessment, two different practical antenna topologies are selected. One antenna is designed to operate in the lower sub-band, while the other is designed to operate in the upper sub-band.

By far the most popular antenna topologies used today are the classical dipole and rectangular patch. These topologies have the advantage of compact size and low cost. However, these antennas belong to the class of resonant antennas (being $\lambda / 4$ or $\lambda / 2$ in size), which are narrowband and unsuitable for UWB applications. The bandwidth of narrow band antennas is usually expressed as a percent:

$$
B_{p}=\frac{f_{U}-f_{L}}{f_{C}} \times 100 \%
$$

Bandwidth for broadband antennas is usually expressed as a ratio given by:

$$
B_{r}=\frac{f_{U}}{f_{L}}
$$

Where, $f_{U}, f_{L}$, and $f_{C}$ represent the upper, lower, and center frequencies of operation, respectively. Classical half-wave dipoles have bandwidths below $20 \%$, while conventional patch designs yield bandwidths as low as a few percent [14]. There are techniques that allow for increasing the bandwidths of dipole and patch antennas. Dipole antennas can be made more broadband by increasing their cross-sectional area [23]. Patches can be built on thicker substrates, stacked, or resistively loaded to make the input impedance less sensitive to changes in frequency [24] [25]. These techniques have the effect of reducing 
the $\mathrm{Q}$ of the antenna at the cost of lower radiation efficiency. However, an analysis by McLean [26] shows that there is a fundamental limit to how much the bandwidth can be increased. It is clear that thick classical dipoles and patches cannot succeed in meeting the bandwidth requirements of UWB.

Because of the extensive research conducted in the field of antennas in the last century, antenna topologies have been invented that are inherently broadband. Horn, helical, biconical, discone and spiral antennas utilize materials with smooth boundaries to reduce the frequency variation of the input impedance [14]. These belong to the class of traveling wave antennas having dimensions of several $\lambda \mathrm{s}$

Figure 2.6 shows a double ridged pyramidal horn antenna. Horns are travelling wave



Figure 2.6: Double ridged horn antenna

antennas that have a narrow pencil beam radiation pattern and are popular in the microwave region above $1 \mathrm{GHz}$. Below this frequency the size of the horn becomes excessive. Horns provide high gain, low VSWR, wide bandwidth and are relatively easy to design and build. Horns are often used as benchmark antennas because of their wide bandwidth and stable radiation pattern. However, their size and awkward non-planar shape make 
them unsuitable for a compact UWB antenna implementation.

The helix of Figure 2.7 is a broadband antenna made by simply winding a wire into a helical shape. The helix can be operated in two modes: the normal and the axial mode.

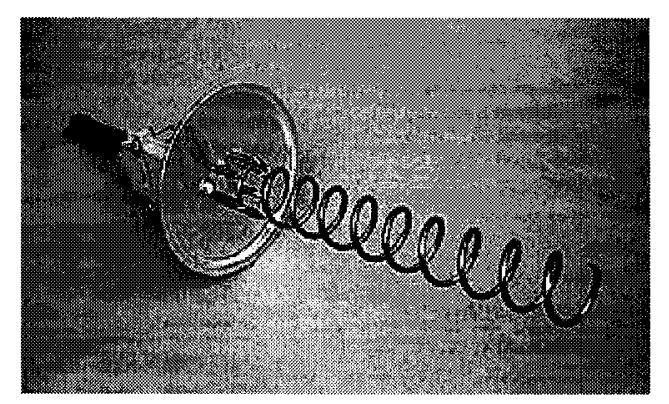

Figure 2.7: Helical antenna

The normal occurs when the helix diameter (and circumference) is small compared to a wavelength and yields a dipole like radiation pattern. When the helix circumference is on the order of a wavelength, the axial mode will result yielding a pencil beam radiation pattern along the axis of the antenna. Helical antennas are used in satellite communications and in some hand-held cellular radios. But because of their size, need for an orthogonal ground plane and non-planar topology, they are unsuitable for a compact UWB antenna implementation.

As discussed earlier, the bandwidth of a simple dipole can be increased by using a thicker wire. A biconical antenna results when a thick wire dipole is flared outwards. The ideal biconical antenna is infinite in length and acts as a perfect guide for spherical waves. The radiation pattern is similar to that of a simple monopole or dipole of the same length. A practical implementation of this antenna is called the finite biconical antenna. Its performance is poorer because the ends of the cone cause reflections that set up standing waves, leading to a complex input impedance [14]. If one arm of the finite biconical 
antenna is replaced by a ground plane a discone antenna results. This antenna can be designed for broadband impedance performance, while maintaining acceptable pattern behavior with frequency. However, its main application is as a broadcast antenna and its size does not make it a good candidate for a compact UWB antenna implementation.

Spiral antennas belong to the class of frequency-independent antennas and are characterized by an exactly or nearly self-complementary shape. This characteristic yields

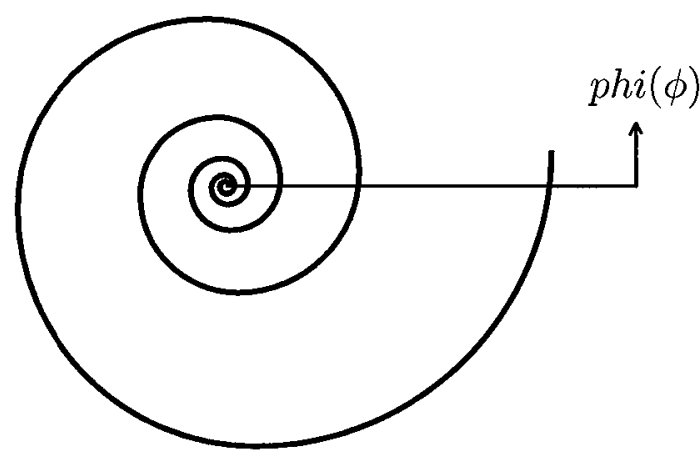

Figure 2.8: Equiangular spiral antenna

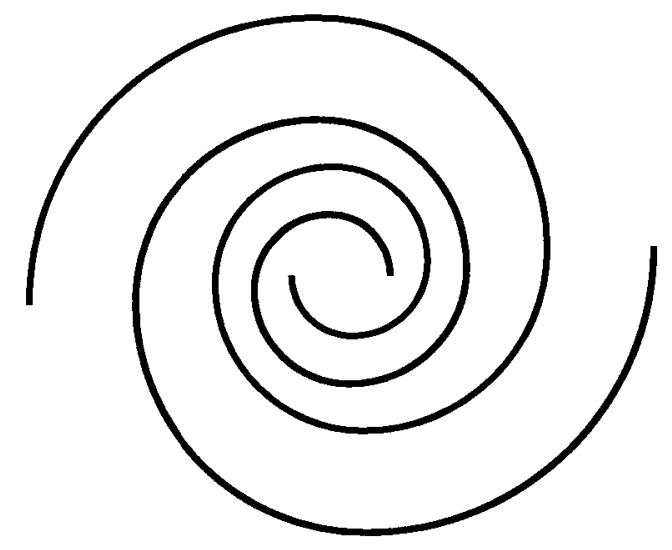

Figure 2.9: Equiangular spiral antenna

extremely wide bandwidths. Currents travel along the arms of the spiral until they finally dissipate at the ends. Radiation occurs in the regions of the spiral that are one wavelength in circumference and are called active regions [14]. The single-ended and balanced forms of the equiangular spiral antenna are shown in Figures 2.8 and 2.9, respectively. The spiral is represented by the generating formula:

$$
r=r_{0} e^{a \phi}
$$

Where, $r_{0}$ is the radius for $\phi=0$ and $a$ is a constant controlling the flare rate of the spiral. This antenna can be designed to radiate either left handed or right handed circular 
polarizations in a bidirectional pattern.

There are two important disadvantages to spiral antennas that make them a poor choice for a compact UWB antenna implementation. Namely, the requirement of a wideband balun to feed the two arms $180^{\circ}$ out-of-phase and the poor radiation efficiency that results if absorbing material is used to create a unidirectional beam.

Log periodic antennas are characterized by a structural geometry such that its impedance and radiation characteristics repeat periodically as the logarithm of frequency. The frequency limits of the log periodic dipole array are set by the frequencies where the largest and smallest dipoles are a quarter-wavelength long [14]. Although these antennas have a very broad bandwidth and nearly identical patterns over a 10:1 range [27], they have critical disadvantages. Log-periodic antennas have been found to be very dispersive in the time domain [18]. The differently scaled components of these antennas radiate different frequency components - introducing delays and the result is a chirp-like dispersive waveform. This makes log-periodic antennas unsuitable for any UWB application.

Recently, fractal antennas have gained a lot of interest because of their small size and multi-band characteristics all without the requirement for extra components [28]. Perhaps the most well known fractal antenna is the Sierpinski monopole of Figure 2.10. The shape is created by taking an initial structure called a generator (in this case a triangle) and replicating it many times at different scales, positions and directions, to grow the final fractal structure [29]. The fractal technique can be applied to miniaturize other conventional antennas such as dipoles, loops and patches [30]. The fractal antenna operates on the same principle as log periodic arrays. That is, when an electromagnetic 


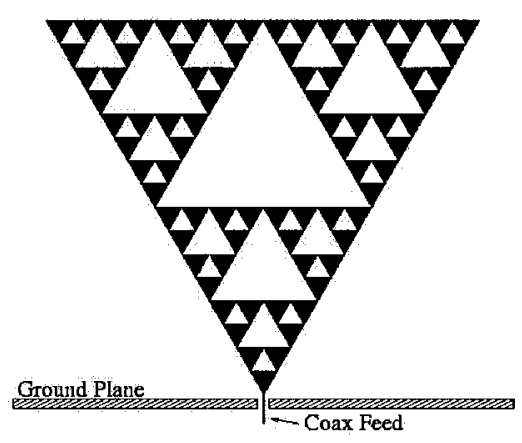

Figure 2.10: Sierpinski monopole

wave is fed through the tip of the antenna it starts to propagate over the structure and is radiated when it encounters a cluster comparable in size to its wavelength [29]. Because of the lack of information on the impulse response of fractal antennas we can only hypothesize that, like standard log-periodic antennas, their time domain performance will be unsuitable for UWB applications.

\subsubsection{UWB Antenna Guidelines}

Summarized below, are guidelines for UWB antenna design based on the discussion above and the good time domain characteristics of the UWB antennas presented in Chapter 4.

- Traveling wave antennas or antennas having low $\mathrm{Q}$ can be very broadband and offer good time domain performance.

- Antennas with multiple resonances such as log periodic or fractal antennas, although broadband, introduce a large variation in the group delay and offer unsuitable time domain performance [18].

- Using low loss materials maximizes radiation efficiency. 
- Linearly polarized transmit and receive antennas are the simplest to implement in a compact planar package.

- Antennas incorporating tapers or rounded edges tend to give broad bandwidths because surface currents have a smooth path to follow. [31]

\subsection{Conclusion}

In this chapter UWB system considerations, basic antenna parameters and requirements for UWB have been presented. Then a variety of antennas have been critically reviewed. Even after the application of special broadband techniques, the basic dipole/monopole and patch designs were found to be too narrowband for UWB applications. Horn antennas can satisfy the bandwidth requirement of UWB but their size and bulkiness rules them out as a practical option. Similarly, a wide-band helix antenna is unsuitable for a compact UWB application because of its awkward non-planar shape and requirement for an orthogonal ground plane. Biconical and discone antennas, although broadband, are too large to be considered as a good candidate for a compact UWB implementation. Single-ended and differential spiral antennas can be made compact and broadband enough for UWB, however they suffer from poor radiation efficiency due to the need for absorbing material and resistive loading. Furthermore, radiating circular polarization is not the traditional form used in short range wireless communications. Finally, log-periodic and fractal antennas cannot be considered as practical UWB antennas because of their dispersive response in the time domain. Novel antenna designs will be developed in Chapter 4 as candidates for compact and practical UWB applications with circuit integration possibilities. 


\section{Chapter 3}

\section{The Finite Difference Time Domain (FDTD) Technique}

In this chapter a FDTD-based electromagnetic simulator, developed at CRC, is presented. The software was created to overcome some limitations in the areas of feed mechanisms and to be a lower cost and more flexible alternative to commercially available FDTD simulators. An introduction to the procedure for applying the FDTD technique to Maxwell's time domain curl equations is given to provide insight into the operation of the program. Important FDTD analysis considerations and the overall program structure will be discussed before a conventional microstrip patch antenna is studied.

\subsection{Introduction to Maxwell's Equations and the Yee Algorithm}

The FDTD method solves Maxwell's curl equations directly in the time domain by converting them into finite-difference equations. These are then solved in a time marching sequence by alternately calculating the electric and magnetic field components on an interlaced spatial grid [32]. The main advantages of the FDTD technique are listed below: 
1. Broadband response predictions with a single excitation.

2. Arbitrary 3D model geometries.

3. The method requires $\mathrm{O}(\mathrm{N})$ storage for $\mathrm{N}$ unknowns.

4. This technique does not perform matrix inversions, which are costly in terms of computing resources.

5. When the geometric and material description of the model become complex, the algorithm complexity remains low.

6. Ability to model materials of any conductivity and frequency dependance.

With the advancement of computer resources the FDTD technique has become increasingly popular in the design of antennas for applications ranging from simple microstrip antennas to complex phased-arrays [33] [34]. Unlike frequency domain based electromagnetic simulators such as HFSS, the FDTD technique also lends itself well to the study of UWB antennas because it provides a method to evaluate waveform distortion directly.

The algorithm introduced by K. Yee in 1966 [35] is the foundation of FDTD electromagnetic field analysis. To simulate the wave propagation in three dimensions, Yee's algorithm replaces Maxwell's equations with a system of finite difference equations. Maxwell's equations needed to specify the field behavior over time are:

$$
\begin{gathered}
\nabla \times \mathbf{E}=-\frac{\partial \mathbf{B}}{\partial t}-\mathbf{J}_{m} \\
\nabla \times \mathbf{H}=\frac{\partial \mathbf{D}}{\partial t}+\mathbf{J}_{e}
\end{gathered}
$$




$$
\begin{gathered}
\mathbf{D}=\varepsilon \mathbf{E} ; \mathbf{B}=\mu \mathbf{H} \\
\mathbf{J}=\sigma \mathbf{E} ; \mathbf{M}=\sigma^{*} \mathbf{H}
\end{gathered}
$$

Where, the asterisk $(*)$ denotes a complex parameter, $\mathbf{E}$ is the electric field, $\mathbf{D}$ is the electric flux density, $\mathbf{H}$ is the magnetic field, $\mathbf{B}$ is the magnetic flux density, $\mathbf{J}_{e}$ is the electric conduction current density and $\mathbf{J}_{m}$ is the equivalent magnetic conduction current density.

Substitution of Equations 3.3 and 3.4 into 3.1 and 3.2, respectively, yields Maxwell's curl equations in linear, isotropic, non-dispersive and lossy materials:

$$
\begin{gathered}
\frac{\partial \mathbf{H}}{\partial t}=-\frac{1}{\mu}\left(\nabla \times \mathbf{E}+\sigma^{*} \mathbf{H}\right) \\
\frac{\partial \mathbf{E}}{\partial t}=\frac{1}{\varepsilon}(\nabla \times \mathbf{H}-\sigma \mathbf{E})
\end{gathered}
$$

Where, $\mu$ is the magnetic permeability, $\varepsilon$ is the electric permittivity and $\sigma$ is the electric conductivity.

Writing the vector components of the curl operators of Equations 3.5 and 3.6 in cartesian coordinates yields the following system of six coupled scalar equations:

$$
\begin{aligned}
& \frac{\partial H_{x}}{\partial t}=\frac{1}{\mu}\left(\frac{\partial E_{y}}{z}-\frac{\partial E_{z}}{y}-\sigma^{*} H_{x}\right) \\
& \frac{\partial H_{y}}{\partial t}=\frac{1}{\mu}\left(\frac{\partial E_{z}}{x}-\frac{\partial E_{x}}{z}-\sigma^{*} H_{y}\right) \\
& \frac{\partial H_{z}}{\partial t}=\frac{1}{\mu}\left(\frac{\partial E_{x}}{y}-\frac{\partial E_{y}}{x}-\sigma^{*} H_{z}\right) \\
& \frac{\partial E_{x}}{\partial t}=\frac{1}{\varepsilon}\left(\frac{\partial H_{z}}{y}-\frac{\partial H_{y}}{z}-\sigma E_{x}\right) \\
& \frac{\partial E_{y}}{\partial t}=\frac{1}{\varepsilon}\left(\frac{\partial H_{x}}{z}-\frac{\partial H_{z}}{x}-\sigma E_{y}\right) \\
& \frac{\partial E_{z}}{\partial t}=\frac{1}{\varepsilon}\left(\frac{\partial H_{y}}{x}-\frac{\partial H_{x}}{y}-\sigma E_{z}\right)
\end{aligned}
$$

By applying the Yee algorithm, Equations 3.7 and 3.8 can be represented in a discrete 
form. The Yee algorithm employs a second-order approximation resulting from a Taylor series expansion given by:

$$
\frac{\partial f}{\partial x}\left(x_{0}\right)=\frac{f\left(x_{0}+\frac{\Delta}{2}\right)-f\left(x_{0}-\frac{\Delta}{2}\right)}{\Delta}+O\left(\Delta^{2}\right)
$$

The symbol delta $(\Delta)$ represents a small increment in space or time. As illustrated in Figure 3.1, the Yee algorithm centers its $\mathbf{E}$ and $\mathbf{H}$ components in three-dimensional space so that every $\mathbf{E}$ component is surrounded by four circulating $\mathbf{H}$ components and every $\mathbf{H}$ component is surrounded by four circulating $\mathrm{E}$ components [36]. The computational

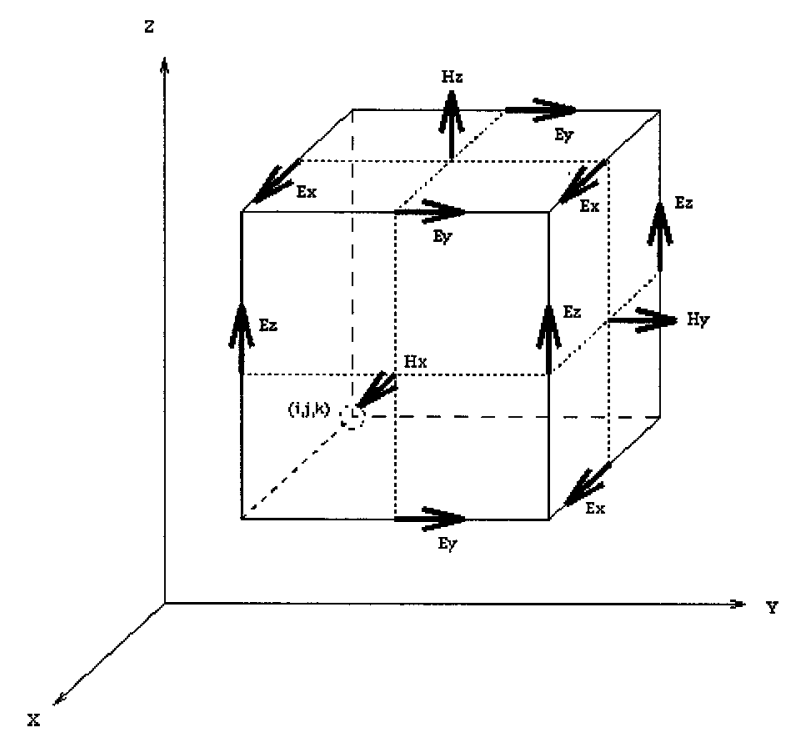

Figure 3.1: The FDTD unit cell

domain is constructed by stacking the Yee unit cells into a large problem space. A stepped or 'staircase' approximation is made of the structure to be simulated. The space resolution and the accuracy of the results are determined by the size of the Yee unit cell. 
Equations 3.7 and 3.8 are also referred to as update equations because all the $\mathbf{E}$ computations are completed and stored in memory for a particular time point using $\mathbf{H}$ data previously stored in memory. Then all the $\mathbf{H}$ computations in the problem space are completed and stored in memory from the $\mathbf{E}$ data just computed. The cycle continues until the maximum number of time steps is reached. By taking the discrete coupled-scalar equations and using Yee's notation, a point in the rectangular problem space is denoted as:

$$
(i, j, k)=(i \Delta x, j \Delta y, k \Delta z)
$$

While, any function $u$ of space and time evaluated at a specific time and location in the problem space is denoted as:

$$
u(i \Delta x, j \Delta y, k \Delta z, n \Delta t)=\left.u\right|_{i, j, k} ^{n}
$$

Where, $\Delta x, \Delta y, \Delta z$ are the lattice space increments in the $\mathrm{x}, \mathrm{y}$ and $\mathrm{z}$ directions and $\mathrm{i}, \mathrm{j}$ and $\mathrm{k}$ are integers ranging from 0 to $\mathrm{n}$, respectively. By applying the above notations and the discrete approximation of Equation 3.9 we obtain the following explicit time-stepping relations for the $\mathrm{E}$ and $\mathrm{H}$ fields in a source free lossy media:

$$
\begin{aligned}
& \left.E_{x}\right|_{i, j, k} ^{n+1}=\left.\left.C_{a}\right|_{i, j, k} E_{x}\right|_{i, j, k} ^{n}+ \\
& \left.C_{b}\right|_{i, j, k}\left[\left(\frac{\left.H_{z}\right|_{i, j+1 / 2, k} ^{n+1 / 2}-\left.H_{z}\right|_{i, j-1 / 2, k} ^{n+1 / 2}}{\Delta y}\right)-\left(\frac{\left.H_{y}\right|_{i, j, k+1 / 2} ^{n+1 / 2}-\left.H_{y}\right|_{i, j, k-1 / 2} ^{n+1 / 2}}{\Delta z}\right)\right] \\
& \left.E_{y}\right|_{i, j, k} ^{n+1}=\left.\left.C_{a}\right|_{i, j, k} E_{y}\right|_{i, j, k} ^{n}+ \\
& \left.C_{b}\right|_{i, j, k}\left[\left(\frac{\left.H_{x}\right|_{i, j, k+1 / 2} ^{n+1 / 2}-\left.H_{x}\right|_{i, j, k-1 / 2} ^{n+1 / 2}}{\Delta z}\right)-\left(\frac{\left.H_{z}\right|_{i+1 / 2, j, k} ^{n+1 / 2}-\left.H_{z}\right|_{i-1 / 2, j, k} ^{n+1 / 2}}{\Delta x}\right)\right]
\end{aligned}
$$




$$
\begin{aligned}
& \left.E_{z}\right|_{i, j, k} ^{n+1}=\left.\left.C_{a}\right|_{i, j, k} E_{z}\right|_{i, j, k} ^{n}+ \\
& \left.C_{b}\right|_{i, j, k}\left[\left(\frac{\left.H_{y}\right|_{i+1 / 2, j, k} ^{n+1 / 2}-\left.H_{y}\right|_{i-1 / 2, j, k} ^{n+1 / 2}}{\Delta x}\right)-\left(\frac{\left.H_{x}\right|_{i, j+1 / 2, k} ^{n+1 / 2}-\left.H_{x}\right|_{i, j-1 / 2, k} ^{n+1 / 2}}{\Delta y}\right)\right] \\
& \left.C_{a}\right|_{i, j, k}=\frac{1-\frac{\sigma_{i, j, k} \Delta t}{2 \varepsilon_{i, j, k}}}{1+\frac{\sigma_{i, j, k} \Delta t}{2 \varepsilon_{i, j, k}}},\left.C_{b}\right|_{i, j, k}=\frac{\frac{\Delta t}{\varepsilon_{i, j, k}}}{1+\frac{\sigma_{i, j, k} \Delta t}{2 \varepsilon_{i, j, k}}} \\
& \left.H_{x}\right|_{i, j, k} ^{n+1 / 2}=\left.\left.D_{a}\right|_{i, j, k} H_{x}\right|_{i, j, k} ^{n-1 / 2}+ \\
& \left.D_{b}\right|_{i, j, k}\left[\left(\frac{\left.E_{y}\right|_{i, j, k+1 / 2} ^{n}-\left.E_{y}\right|_{i, j, k-1 / 2} ^{n}}{\Delta z}\right)-\left(\frac{\left.E_{z}\right|_{i, j+1 / 2, k} ^{n}-\left.E_{z}\right|_{i, j-1 / 2, k} ^{n}}{\Delta y}\right)\right] \\
& \left.H_{y}\right|_{i, j, k} ^{n+1 / 2}=\left.\left.D_{a}\right|_{i, j, k} H_{y}\right|_{i, j, k} ^{n-1 / 2}+ \\
& \left.D_{b}\right|_{i, j, k}\left[\left(\frac{\left.E_{z}\right|_{i+1 / 2, j, k} ^{n}-\left.E_{z}\right|_{i-1 / 2, j, k} ^{n}}{\Delta x}\right)-\left(\frac{\left.E_{x}\right|_{i, j, k+1 / 2} ^{n}-\left.E_{x}\right|_{i, j, k-1 / 2} ^{n}}{\Delta z}\right)\right] \\
& \left.H_{z}\right|_{i, j, k} ^{n+1 / 2}=\left.\left.D_{a}\right|_{i, j, k} H_{z}\right|_{i, j, k} ^{n-1 / 2}+ \\
& \left.D_{b}\right|_{i, j, k}\left[\left(\frac{\left.E_{x}\right|_{i, j+1 / 2, k} ^{n}-\left.E_{x}\right|_{i, j-1 / 2, k} ^{n}}{\Delta y}\right)-\left(\frac{\left.E_{y}\right|_{i+1 / 2, j, k+1 / 2} ^{n}-\left.E_{y}\right|_{i-1 / 2, j, k} ^{n}}{\Delta x}\right)\right] \\
& \left.D_{a}\right|_{i, j, k}=\frac{1-\frac{\sigma_{i, j, k}^{*} \Delta t}{2 \mu_{i, j, k}}}{1+\frac{\sigma_{i, j, k}^{*} \Delta t}{2 \mu_{i, j, k}}},\left.\quad D_{b}\right|_{i, j, k}=\frac{\frac{\Delta t}{\mu_{i, j, k}}}{1+\frac{\sigma_{i, j, k}^{*} \Delta t}{2 \mu_{i, j, k}}}
\end{aligned}
$$

Equations 3.12 to 3.19 represent the core of the FDTD time-stepping algorithm. The half 
time steps indicate that $\mathbf{E}$ and $\mathbf{H}$ are alternately calculated in order to achieve centered differences for the time derivatives. This system of equations is directly implementable on a computer. However, before time-stepping begins each Yee cell must be assigned an appropriate permittivity, permeability and conductivity.

\subsection{Numerical Stability and Dispersion}

The cell size and time increment must be determined before the difference equations presented in the previous section can be computed. The cell size is usually determined first based upon the physical dimensions of the structure to be analyzed and the available computational resources. Then the time increment is determined such that numerical stability is achieved [36].

\subsubsection{Stability}

The choice of the time increment $\Delta t$ has an important impact on the stability of the FDTD algorithm. Without an upper bound, numerical instability can be encountered that causes the computed results to increase uncontrollably as time-marching continues. Courant's stability criterion (3.20) provides an upper bound for the time increment $\Delta t$. When satisfied, it ensures numerical stability.

$$
\Delta t \leq \frac{1}{c \sqrt{\left(\frac{1}{\Delta x}\right)^{2}+\left(\frac{1}{\Delta y}\right)^{2}+\left(\frac{1}{\Delta z}\right)^{2}}}
$$

Where, $\mathrm{c}$ is the maximum velocity of the wave propagating in the media, usually the speed of light in free space. 


\subsubsection{Dispersion}

The FDTD algorithms can cause non-physical dispersion of simulated waves in the computational domain. The wavelength, direction of propagation and grid sizing can cause variations in the phase velocity from that of $c$ of numerical wave modes. Dispersion can cause the distorion of a pulse shape and is especially a problem for electrically large structures. The choice of the lattice increment, $\Delta$, has an important impact on numerical stability. In general, the lattice increment in any of the three cartesian directions should be less than $\lambda_{u} / 20$ [36], where $\lambda_{u}$ corresponds to the wavelength of the highest significant frequency content in the excitation frequency spectrum. This ensures that the uncertainty in the computed field magnitude is less than $2 \%$. At the cost of increasing the computational time, dispersion can be minimized by increasing the resolution of the FDTD lattice.

\subsection{Excitation Methods}

An electromagnetic wave excitation in a FDTD mesh can take any form. However, wave excitations are classified as either narrowband, bandpass or broadband. Equation 3.21 is an example of a narrowband excitation, which is a continuous sinusoidal wave of frequency $f_{0}$ that is switched on at time $\mathrm{t}=0$ or the time index $\mathrm{n}=0$. The simulation terminates when steady state is achieved.

$$
\begin{aligned}
& \left.E_{z}\right|_{i, j, k} ^{n}=E_{0} \sin \left(2 \pi f_{0} n \Delta t\right) \\
& t=n \Delta t
\end{aligned}
$$


In this work a broadband pulse excitation is used. A broadband source waveform is defined by Equation 3.22. This waveform is of the form of a Gaussian pulse that is truncated in time and has a predefined frequency bandwidth. A short duration Gaussian pulse has a very wide frequency spectrum. Thus, using a single Gaussian excitation allows for broadband response predictions.

$$
\begin{aligned}
& \left.E_{z}\right|_{i, j, k} ^{n}=E_{0} e^{-\left(\frac{t-t_{0}}{T}\right)^{2}} \\
& t_{0}=n_{0} \Delta t
\end{aligned}
$$

The Gaussian pulse should provide relatively high signal levels within the frequency range of interest. The parameters $\mathrm{T}$ and $t_{0}$ should be chosen carefully to obtain the required response. T defines the width of the Gaussian pulse and therefore can be used to provide the desired cutoff frequency. Figure 3.2 shows that the effect of changing $\mathrm{T}$ from 15 ps to $5 \mathrm{ps}$ is an increase in the cutoff frequency.
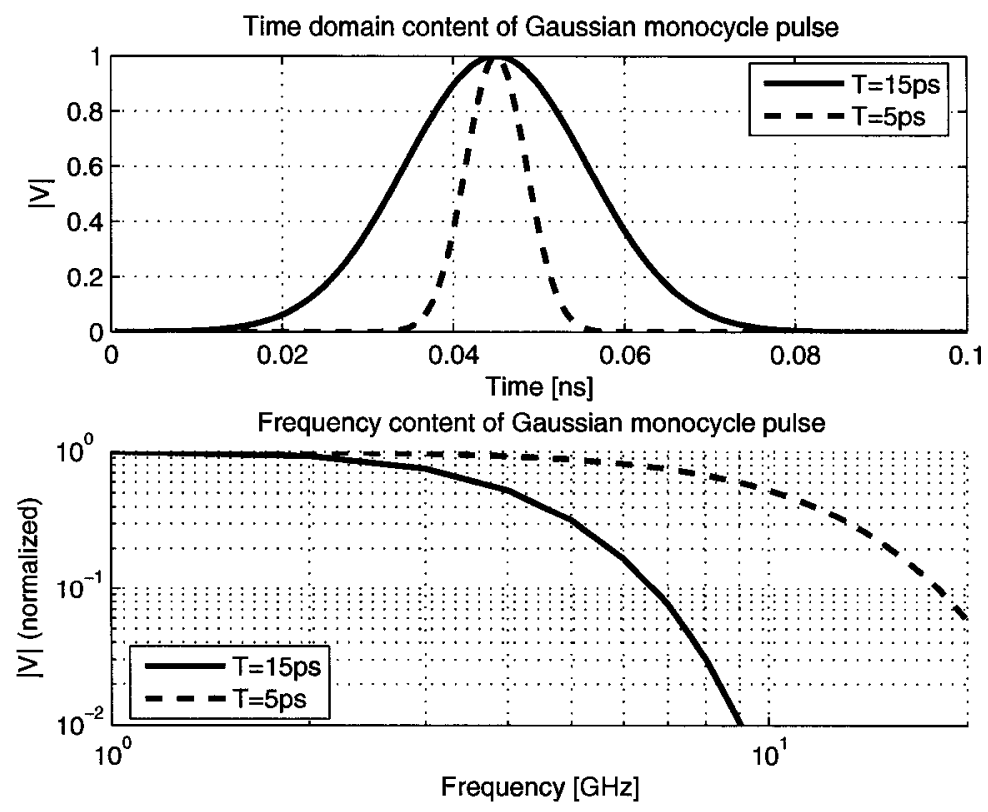

Figure 3.2: Broadband Gaussian Excitation 
The value of the parameter $t_{0}$ is chosen so that the rise time of the pulse is short and smooth. In this work, $t_{0}$ is set to $3 \mathrm{~T}$ so that the pulse is down to $e^{-9}$ of its maximum value at the truncation time $t=0$ or $t=2 t_{0}$. At $t=0$ the fields are assumed to be zero throughout the computational domain. After the first time step $(n=1)$, a vertical electric field, $E_{z}$, is forced in a rectangular region beneath the microstrip line. This has the effect of simulating a voltage source excitation. A perspective view of a microstrip simulation setup is shown in Figure 3.3.

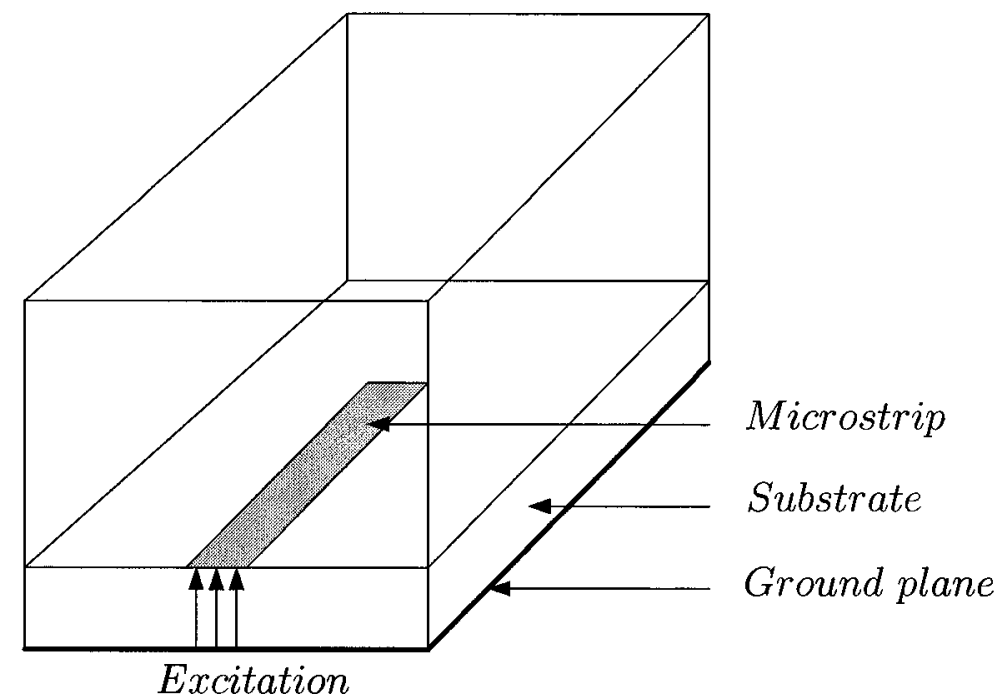

Figure 3.3: Microstrip Excitation

A minimal amount of source distortion is apparent when image theory is used to enforce a magnetic wall (i.e. $\mathbf{H}_{t a n}$ outside the magnetic wall is equal to $-\mathbf{H}_{t a n}$ inside the magnetic wall). Specifying the excitation in this way ensures that only the fundamental mode will travel down the microstrip line in the desired frequency range [33]. 


\subsection{Absorbing Boundary Conditions}

Due to the finite memory of computers, the computational domain must be limited in size. The cubic outer boundary of the computational domain must absorb outgoing waves in such a way that they don't reflect back into the problem space and cause errors. In this work, a first order MUR [37] absorbing boundary condition (ABC) is implemented. It has been shown that this type of ABC is sufficiently accurate and particularly well suited to the microstrip geometry [33].

An estimate of the tangential electric fields at the boundary is needed to allow the Yee algorithm to operate. There are two Mur estimates for the value of the fields at the boundary which are first order and second order accurate. The first order Mur estimate, which is used in this work, looks back one step in time and one cell location in space. This estimate assumes that the waves are normally incident at the outer walls of the problem space. This assumption forces the tangential fields at the outer boundaries to obey the one-dimensional wave equation. Two sets of equations are used at each face of the outer boundary, representing estimates for the tangential $\mathbf{E}$ field components.

By considering the top face of the Yee mesh $(\mathrm{x}=\mathrm{i} \Delta x, \mathrm{y}=\mathrm{j} \Delta y, \mathrm{z}=\mathrm{LIMZ})$, the first order Mur estimate is:

$$
\begin{aligned}
& \left.E_{x}\right|_{i, j, L I M Z} ^{n+1}=\left.E_{x}\right|_{i, j, L I M Z-1} ^{n}+\frac{c \Delta t-\Delta z}{c \Delta t-\Delta z}\left(\left.E_{x}\right|_{i, j, L I M Z-1} ^{n+1}-\left.E_{x}\right|_{i, j, L I M Z} ^{n}\right) \\
& \left.E_{y}\right|_{i, j, L I M Z} ^{n+1}=\left.E_{y}\right|_{i, j, L I M Z-1} ^{n}+\frac{c \Delta t-\Delta z}{c \Delta t-\Delta z}\left(\left.E_{y}\right|_{i, j, L I M Z-1} ^{n+1}-\left.E_{y}\right|_{i, j, L I M Z} ^{n}\right)
\end{aligned}
$$

By considering the bottom face of the Yee mesh $(\mathrm{x}=\mathrm{i} \Delta x, \mathrm{y}=\mathrm{j} \Delta y, \mathrm{z}=0)$, the first order 
Mur estimate is:

$$
\begin{aligned}
& \left.E_{x}\right|_{i, j, 0} ^{n+1}=\left.E_{x}\right|_{i, j, 1} ^{n}+\frac{c \Delta t-\Delta z}{c \Delta t-\Delta z}\left(\left.E_{x}\right|_{i, j, 1} ^{n+1}-\left.E_{x}\right|_{i, j, 0} ^{n}\right) \\
& \left.E_{y}\right|_{i, j, 0} ^{n+1}=\left.E_{y}\right|_{i, j, 1} ^{n}+\frac{c \Delta t-\Delta z}{c \Delta t-\Delta z}\left(\left.E_{y}\right|_{i, j, 1} ^{n+1}-\left.E_{y}\right|_{i, j, 0} ^{n}\right)
\end{aligned}
$$

Similar equations can be derived for the other four faces of the outer boundary based on the Yee mesh. For Mur ABC's, the ability to absorb outgoing waves increases by increasing the distance between the object being modelled and the outer boundary. For good accuracy, the outer boundary should be placed 15 to 20 cells away from the antenna when using a Mur ABC [14].

\subsection{Conductor and Media Interface Treatment}

The antennas considered in this work are planar microstrip structures. That is, they have a conducting ground plane separated from a top metallization by a dielectric substrate. The metallizations are considered to be perfectly conducting and have zero thickness. They are implemented by setting the $x$ and $y$ components of the $\mathbf{E}$ field that lie on the conductors to zero.

The dielectric constant on the interface of two different materials is modelled as an average of their respective dielectric constants as given by Equation 3.25.

$$
\varepsilon_{i n t}=\frac{\varepsilon_{1}+\varepsilon_{2}}{2}
$$

This result is derived by considering a dielectric interface in the xy-plane, separating two media of permittivity $\varepsilon_{1}$ and $\varepsilon_{2}$. The expressions for the tangential electric field 
components $E_{x}$ are shown below:

$$
\begin{aligned}
& \varepsilon_{1} \frac{\partial E_{x}^{1}}{\partial t}=\frac{\partial H_{z}^{1}}{\partial y}-\frac{\partial H_{y}^{1}}{\partial z} \\
& \varepsilon_{2} \frac{\partial E_{x}^{2}}{\partial t}=\frac{\partial H_{z}^{2}}{\partial y}-\frac{\partial H_{y}^{2}}{\partial z}
\end{aligned}
$$

The boundary conditions require that the tangential electric field and the normal derivative of $H_{y}$ must be continuous. Thus, by adding the preceding equations and enforcing the boundary conditions yields the following equation:

$$
\left(\frac{\varepsilon_{1}+\varepsilon_{2}}{2}\right) \frac{\partial E_{x}}{\partial t}=\frac{1}{2}\left(\frac{\partial H_{z}^{1}}{\partial y}+\frac{\partial H_{z}^{2}}{\partial y}\right)-\frac{\partial H_{y}}{\partial z}
$$

Simplification of Equation 3.27 is possible by replacing the average of the $\frac{\partial H_{z}}{\partial y}$ terms with just $\frac{\partial H_{z}}{\partial y}$ since there is only a very small difference between the two $\frac{\partial H_{z}}{\partial y}$ derivatives [38]. This shows that the interface permittivity is just as given in Equation 3.25.

\subsection{Software Development and Antenna Modeling}

In this work a FDTD based simulator was developed at the Communications Research Center (CRC) for the modeling of UWB antennas. MATLAB script was chosen as the programming language for the implementation. Using MATLAB shortens the development time because of the built in functions and tools that can be used. The plotting and graphics tools were especially useful. Furthermore, MATLAB is a language that uses arrays natively - making it well suited to handling the large arrays generated by the FDTD algorithm. The overall program structure is shown in Figure 3.4. The program begins by 


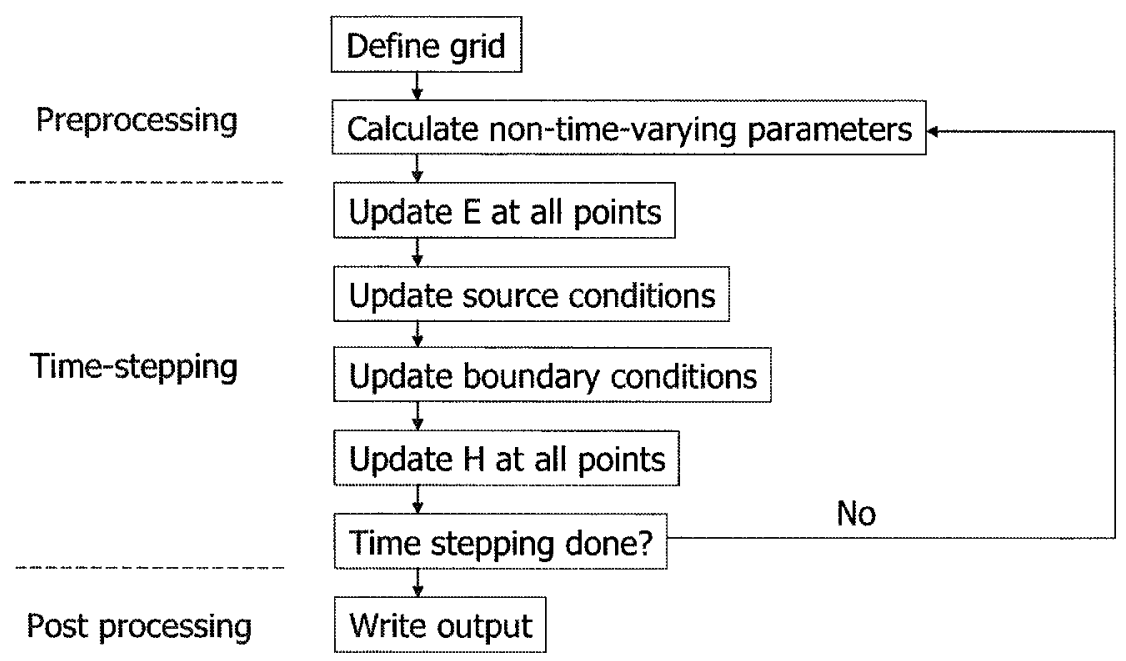

Figure 3.4: FDTD program execution

setting the size of the problem space, defining fundamental constants and object dimensions. Each cube in the mesh is given an appropriate permittivity and conductivity. At this point time stepping begins with the computation of the electric field values in the entire space. Part of the code segment that updates the $\mathbf{E}$ field values is given below:

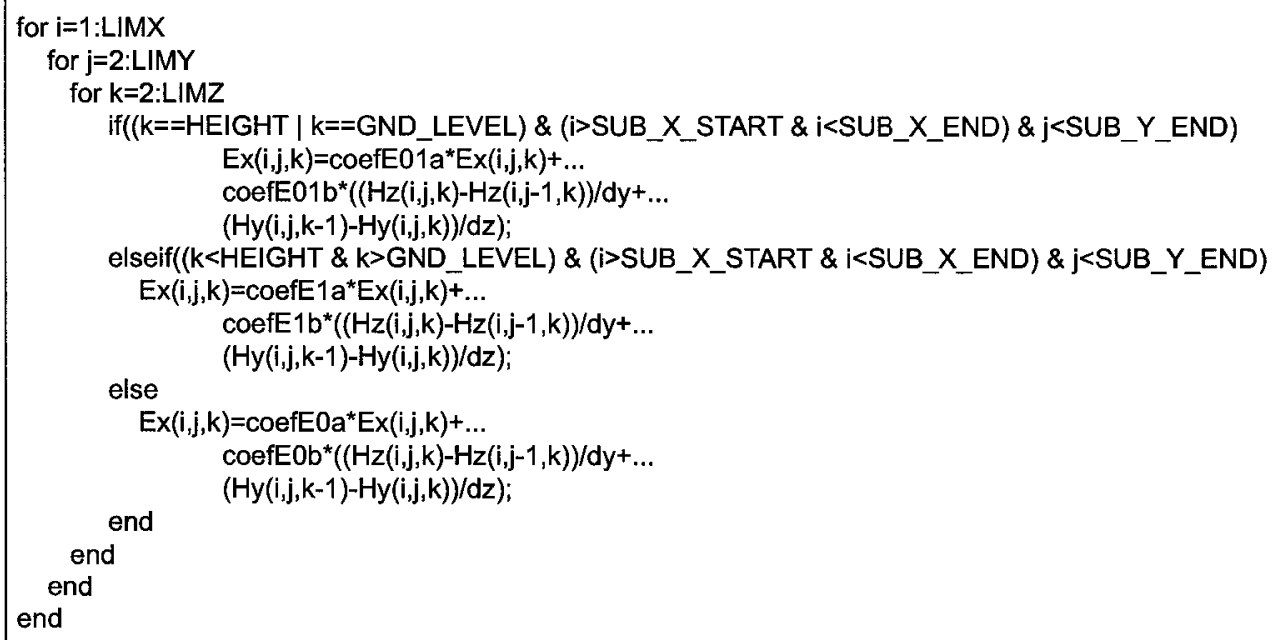

Figure 3.5: FDTD E field code segment

The for loop cycles through the computational domain from bottom to top. The 
variable "HEIGHT" defines the metal layer. Three different materials are encountered during the iteration - dielectric, dielectric-air interface and air. Three if statements are required since each material is defined by a different $\mathbf{E}$ field update equation. The y and z components of the $\mathbf{E}$ field are updated in a similar way.

The next step is to apply the Mur absorbing boundary condition. The code statements that estimate the tangential $\mathbf{E}_{x}$ and $\mathbf{E}_{y}$ components on the top face of the computational domain are:

EX(1:LIMX,2:LIMY,LIMZb)=ex7(1:LIMX,2:LIMY,1)+coefMz0z*(Ex(1:LIMX,2:LIMY,LIMZ)-ex8(1:LIMX,2:LIMY,1)); Ey(1:LIMX,2:LIMY,LIMZb)=ey7(1:LIMX,2:LIMY,1)+coefMzOz*(EY(1:LIMX,2:LIMY, LIMZ)-ey8(1:LIMX,2:LIMY,1));

Figure 3.6: FDTD Mur ABC code segment

Similar code statements are used on the other 5 faces of the outer boundary to estimate the appropriate tangential $\mathbf{E}$ field components there. The last important task in the time stepping loop is to compute the magnetic field components. This step is somewhat easier than the $\mathbf{E}$ field update since the $\mathbf{H}$ field equations are not material dependent. The MATLAB code for the $\mathbf{H}_{x}$ component update equation is:

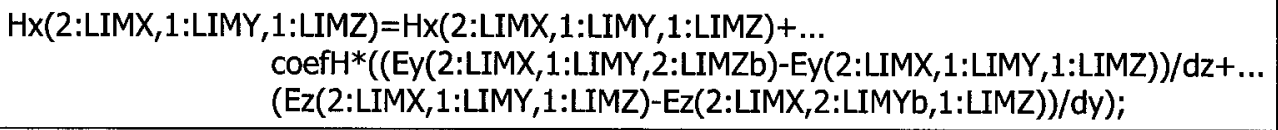

Figure 3.7: FDTD $\mathbf{H}$ field code segment

The electric field underneath the center of each microstrip port is recorded at every time step. It is assumed that the field value is proportional to the voltage. When time stepping terminates the transient results are saved and post-processing can be performed. 
The frequency domain s-parameters and input impedance can be easily calculated from the incident and reflected waves. For the first few nanoseconds of the simulation the data is taken as the input. The rest of the data is the reflection coming back from the object. Equation 3.28 shows that the s-parameters can be obtained by taking the Fourier transform of the reflected waveform divided by the Fourier transform of the incident waveform.

$$
S_{i j}(\omega)=\frac{F\left[V_{j}(t)\right]}{F\left[V_{k}(t)\right]}
$$

To verify the accuracy of the FDTD software, a conventional microstrip patch antenna was simulated and the results were compared to the measured published results by Sheen et al. [33] and those generated by HFSS. The antenna geometry is shown in Figure 3.8. The same Yee mesh parameters were used as in the literature. The total mesh dimensions

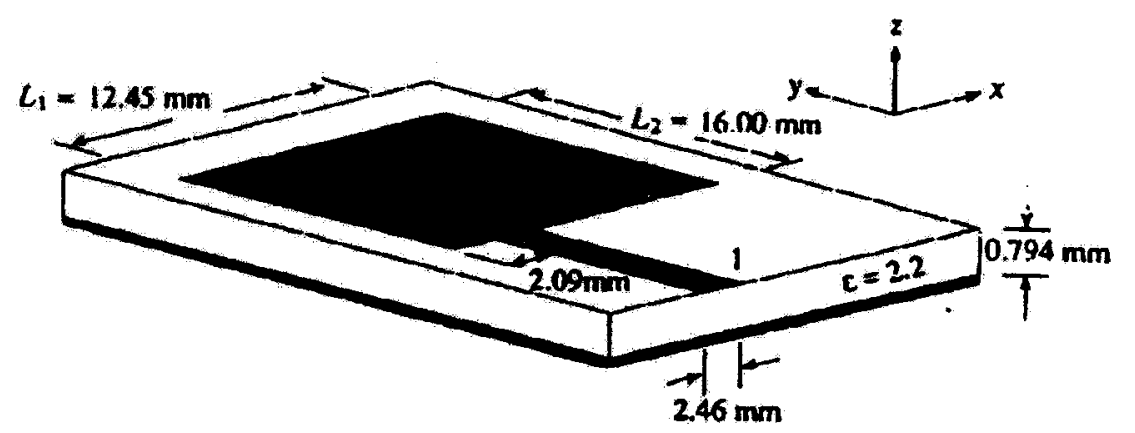

Figure 3.8: Rectangular microstrip patch antenna

are $60 \times 100 \times 16$ in the $\hat{x}, \hat{y}$ and $\hat{z}$ directions, respectively. $\Delta z$ is chosen so that three Yee cells exactly match the thickness of the substrate. $\Delta x$ and $\Delta y$ are chosen such that an integer number match the width and length of the patch. The length of the microstrip line is $50 \Delta y$, while the width is $6 \Delta x$. The space steps used are displayed in Table 3.1 . 
Table 3.1: Sheen Patch Space Steps

\begin{tabular}{|c|c|}
\hline Increment & Value $(\mathrm{mm})$ \\
\hline$\Delta x$ & 0.389 \\
\hline$\Delta y$ & 0.400 \\
\hline$\Delta z$ & 0.265 \\
\hline
\end{tabular}

The time step used is $\Delta t=0.441 \mathrm{ps}$. The Gaussian half-width is $\mathrm{T}=15 \mathrm{ps}$ and the time delay $t_{0}$ is set to be $3 \mathrm{~T}$. The simulation is performed for 8000 time steps. When the FDTD program is executed, the spatial distribution of $E_{z}$ can be viewed in 2 or 3 -dimensions. Figures 3.9, 3.11 and 3.13 show the field distribution, in 2-dimensions, just beneath the metal layer and inside the dielectric substrate. Figures 3.10, 3.12 and 3.14 show the field distribution, in 3-dimensions, just beneath the metal layer of the patch. From these real-time animations, it is possible to see the incident Gaussian pulse travel down the microstrip until it is partially reflected at the patch boundary. The reflected pulse travels back along the microstrip and is absorbed by the Mur ABC on the source wall.

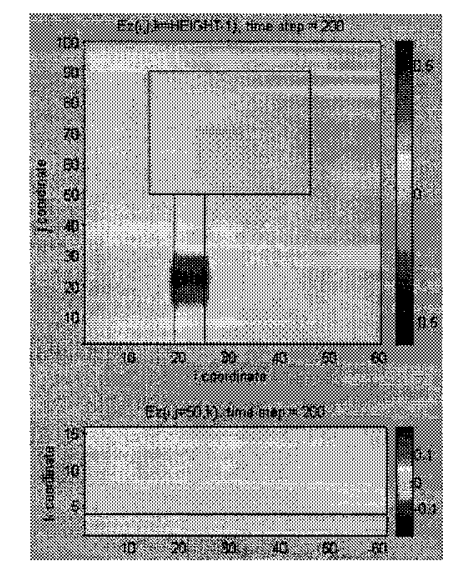

Figure 3.9: Time step: 200

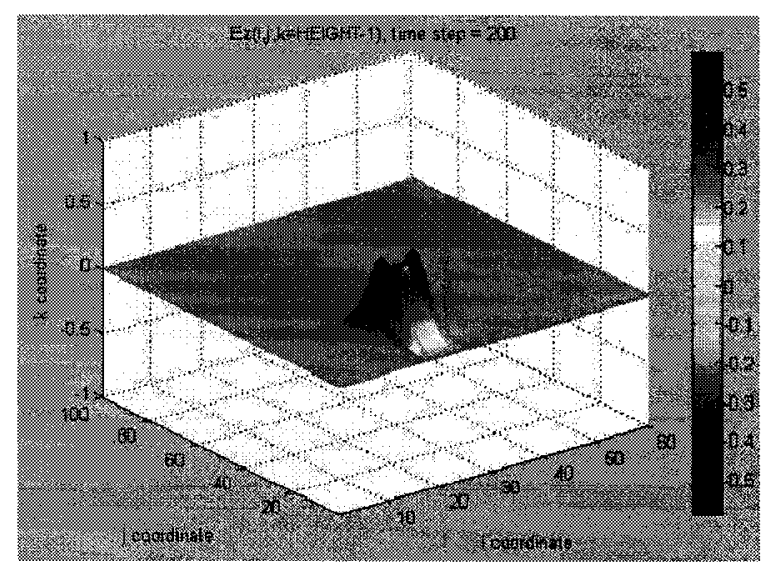

Figure 3.10: Time step: 200

The simulation results are in very good agreement with the published values and are 


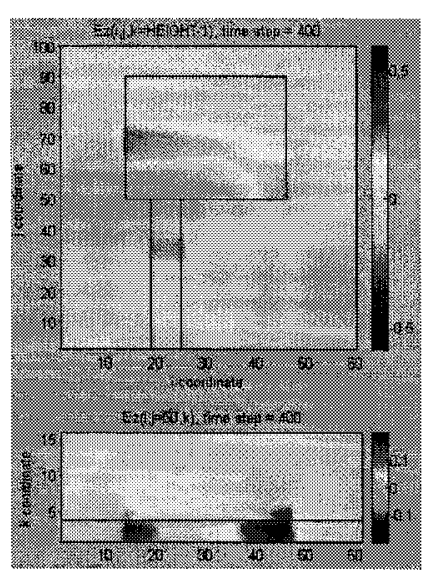

Figure 3.11: Time step: 400



Figure 3.13: Time step: 800



Figure 3.12: Time step: 400

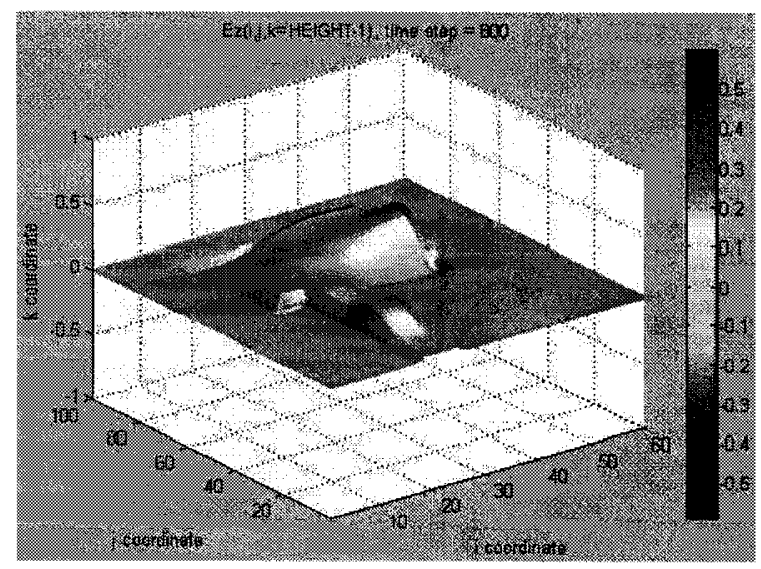

Figure 3.14: Time step: 800

shown in Figure 3.15. The top graph displays the incident and reflected transient data for the first $2.0 \mathrm{~ns}$. The middle graphs displays the return loss in $\mathrm{dB}$ for the antenna. The input impedance for the antenna can be calculated from the s-parameter data with:

$$
Z_{\text {in }}=Z_{0} \frac{1+S_{11} e^{j 2 k L}}{1-S_{11} e^{j 2 k L}}
$$

Where, $\mathrm{k}$ is the wavenumber, $\mathrm{L}$ is the length from the edge of the antenna to the reference plane and $Z_{0}$ is the characteristic impedance of the microstrip line. The bottom graph shows that the simulated and measured input impedance are in good agreement. 

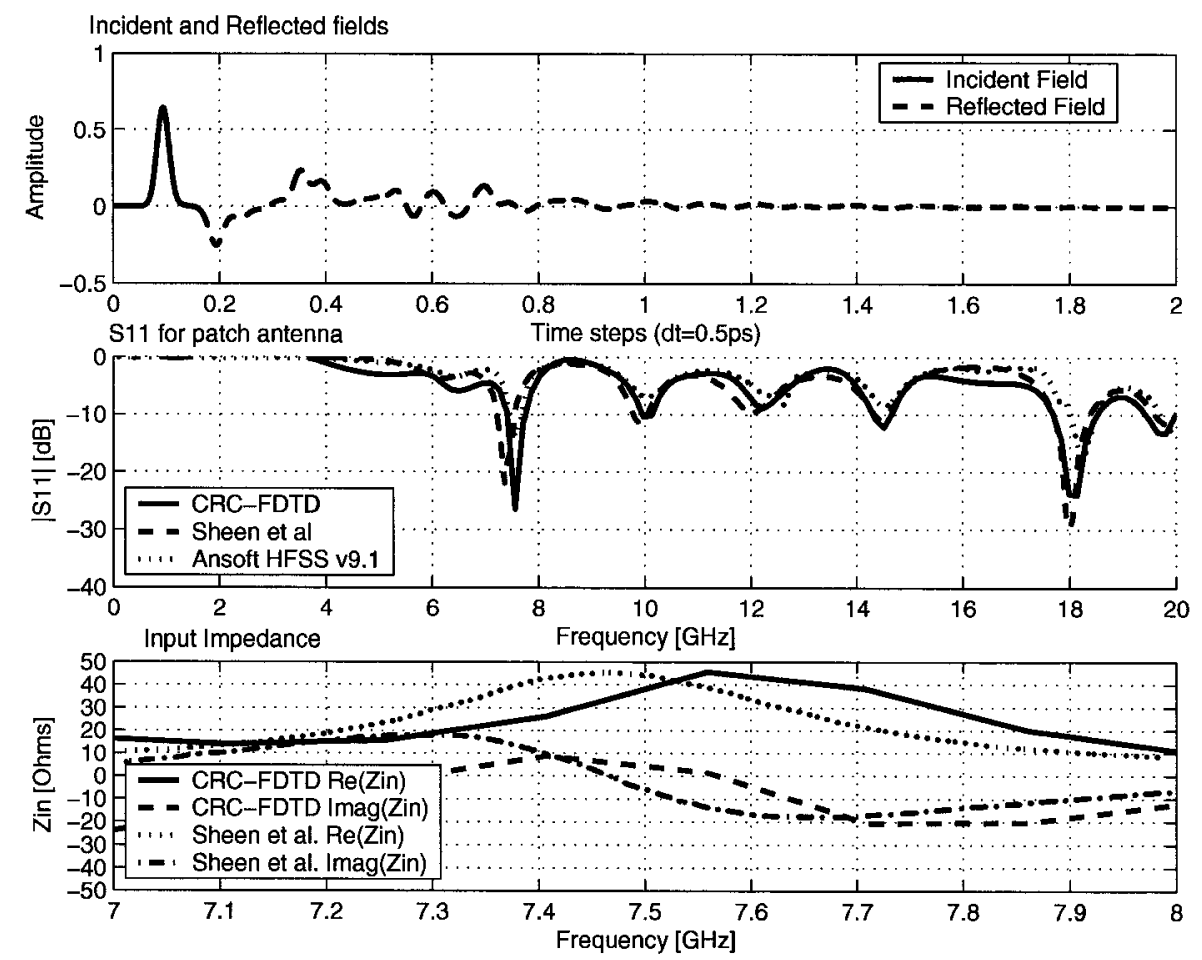

Figure 3.15: Simulation Results for Patch Antenna

\subsubsection{Near Field Probing}

The FDTD technique can be used to understand an antenna's response in the time domain with the use of near field probes. Instead of modeling a two antenna system, which is very computationally expensive, only the electric field intensity radiated by one antenna is sampled. A probe location near the antenna aperture and in the direction of maximum radiation is selected. Importantly, these probes have no effect on the simulation results. The co-polarized component of the electric field is sampled and the results show the waveform that is being radiated by the antenna. Ideally, the radiated waveform should have the same shape as the incident pulse.

In this case, the near field probe was placed 14 unit cells above the center of the patch. 
The co-polarized component of the electric field, $E_{y}$, was sampled and the result is shown below:

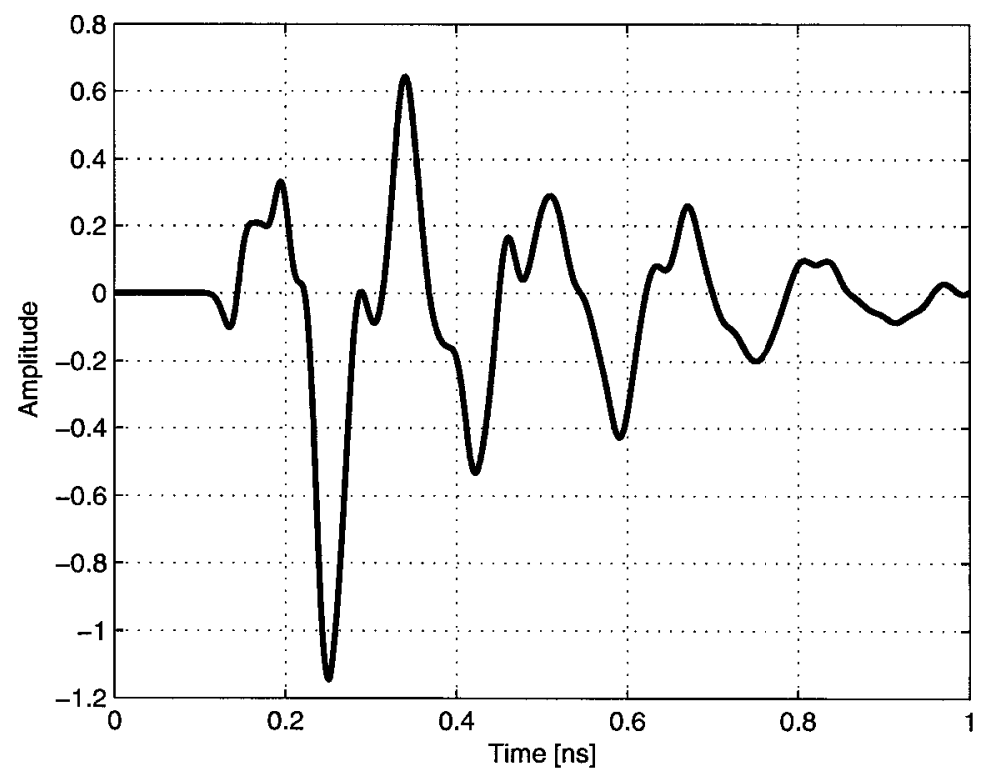

Figure 3.16: Simulated near field probe result for patch antenna

As expected, the plot shows a radiated waveform that is significantly different from the Gaussian pulse that was applied to the antenna input. The narrowband nature of the patch antenna does not allow enough frequency components to be radiated to maintain the integrity of the Gaussian pulse. More broadband antenna structures, such as those studied in Chapter 4, will show improved results.

\subsection{Conclusion}

A study of the FDTD technique and the development of an FDTD-based simulator has been presented in this chapter. Code segments from the actual FDTD program show how 
Maxwell's fundamental electromagnetic equations were implemented in MATLAB. Program attributes and excitation methods relevant to antenna simulation were discussed. The FDTD program generates time domain data that can be transformed into the frequency domain with a Fourier transform. In this way return loss data can be obtained. Near field probing can be used to sample the radiated electric field and assess the level of distortion introduced by the antenna. Finally, a microstrip patch antenna was simulated and the results were in good agreement with the measured published values. This exercise verifies the accuracy of the FDTD software and indicates that it can be used to simulate more complex antenna structures, as will be done in Chapter 4. 


\section{Chapter 4}

\section{Practical UWB Antenna Design}

This chapter provides a detailed discussion on the design of practical UWB antennas based on the observations in Chapter 2. Choosing an antenna topology suitable for a compact UWB application is difficult. All the antennas discussed in Chapter 2 have disadvantages that make them unsuitable as UWB antennas. In this thesis five different antennas were designed, simulated, fabricated and measured, including a triangular monopole with an orthogonal ground plane, a triangular monopole with a partial ground plane and an antipodal Vivaldi antenna. Low cost FR-4 and low-loss LTCC dielectric materials were used in the fabrication of the antennas. The fundamental theories governing the operation of these antennas and the procedures used in their design are presented first followed by the results obtained from extensive simulations performed with HFSS and the FDTD technique.

Ansoft's HFSS is a powerful electromagnetic simulator with built in design capture and many advanced capabilities such as automatic optimizations. However, it is frequency domain based and thus not able to provide time domain information. A FDTD-based electromagnetic simulator was developed to meet this need. As described in Chapter 3, 
frequency domain $\left|S_{11}\right|$ results are generated from the time domain incident and reflected electric fields. These results will be compared to those generated by HFSS and the degree of agreement will be discussed. In addition, HFSS was used to generate radiation pattern plots. Near field probe simulations were performed to obtain an understanding of the time domain performance of each antenna. The complete collection of simulation results will be compared to the measured values in Chapter 5 .

\subsection{Vivaldi Antenna Theory}

Planar Vivaldi antennas have been used in radar-like communications since 1979 [39]. They are a form of tapered slot radiator that support traveling waves. Ground penetrating radar applications [40] and UWB communications studies [41] have shown that this antenna can preserve the shape of transmitted UWB pulses. The basic configuration of this antenna is shown in Figure 4.1. For best performance the flares are exponentially

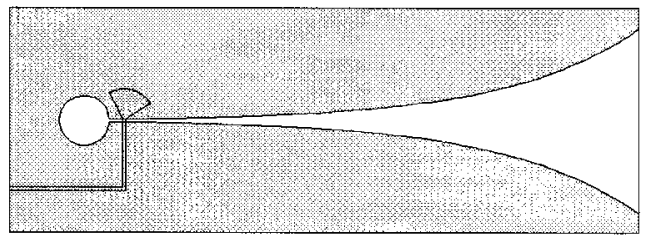

Figure 4.1: Tapered slot Vivaldi with Marchand balun feed

tapered. The performance of this antenna is limited by the need for a wideband balun. Considerable effort is required to give broadband performance since the feed structure is so complicated. Traditionally a Marchand type stripline to slot feed transition is used [42].

More recently, two types of Vivaldi antenna have been developed that overcome these problems by enabling either a simple microstrip or stripline feed method [31] [43]. The 
antipodal Vivaldi antenna (AVA) is a two layer design that uses a microstrip feed, while the balanced antipodal Vivaldi antenna (B-AVA) is a three layer design that uses a stripline feed. Figures 4.2 and 4.3 show an antipodal and balanced antipodal Vivaldi antenna, respectively. While making the feed simpler, these antennas also inherit the good time



Figure 4.2: Antipodal Vivaldi antenna topology (microstrip feed)



Figure 4.3: Balanced antipodal Vivaldi antenna topology (stripline feed)

domain performance of the original tapered slot antenna.

To obtain an understanding of the relative performance of the Vivaldi antenna a time domain $S_{21}$ comparison was made with a log-periodic dipole antenna. The measurements were performed in the frequency domain and then converted into the time domain using an inverse fast Fourier transform. The responses in the time domain, obtained from [44], of these antennas to a Gaussian impulse are shown in Figure 4.4. The log-periodic antenna is dispersive in the time domain because it operates with multiple resonances in the frequency domain. The various sized elements radiate different frequency components of the input signal causing dispersion and late time ringing. It is evident that the Vivaldi antenna has a superior response in the time domain, producing a near perfect Gaussian doublet response with greater efficiency. This antenna can also be fabricated using printed circuit techniques and can be integrated with transceiver electronics. 


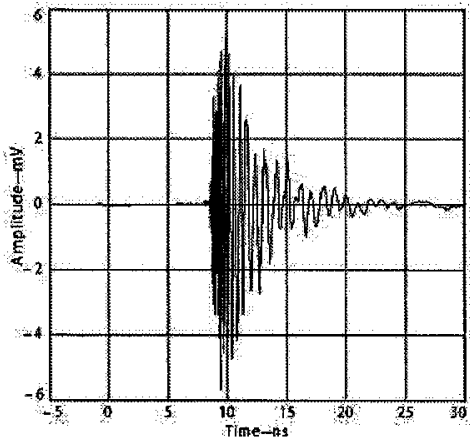

(a)

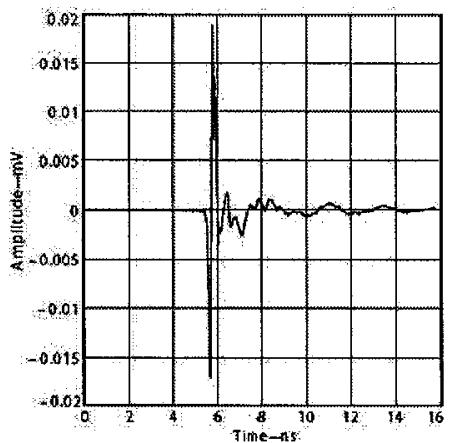

(b)

Figure 4.4: Response in the time domain of (a) log-periodic and (b) antipodal Vivaldi antenna

The AVA is a dual-layer structure composed primarily of three different features: a microstrip feed, a paired-strip middle section and a radiating section. The smooth transition from microstrip to radiating section allows for broadband performance. The transition region is responsible for connecting the highly capacitive feed structure to the inductive radiating section [44]. The radiating section is formed by the metallizations on either side of the substrate that flare in opposite directions, forming a tapered slot.

Adding a third metallization layer to the antipodal Vivaldi structure creates a BAVA. It is a triplate structure with a stripline feed. The stripline transitions smoothly to the radiating section, providing broadband performance. This antenna exhibits a similar input return loss to the antipodal form while showing improved cross-polarization performance [43].

In the literature available today, only limited information on the radiation patterns of the AVA and B-AVA was presented and even less information on the time domain characteristics of these antennas has been shown. 


\subsection{Antipodal Vivaldi Antenna Design and Simula- tion}

Given the promising characteristics of the AVA and B-AVA, these designs will be further pursued in this thesis to fully explore the effects of size, geometry and materials on performance. The first Vivaldi antenna designed was an AVA. It was fabricated at CRC on an FR-4 substrate with gold conductors. The antenna was designed to have an impedance bandwidth from $4.0 \mathrm{GHz}-8.0 \mathrm{GHz}$ to correspond to the available time domain test equipment. The full specifications and material parameters are given in Table 4.1. The physical characteristics of the Vivaldi antenna that affect its operation are the math-

Table 4.1: AVA specifications

\begin{tabular}{|c|c|}
\hline Bandwidth & $4.0 \mathrm{GHz}-8.0 \mathrm{GHz}$ \\
\hline Substrate & $\begin{array}{c}\varepsilon_{r}=\sim 4.4 \\
\text { height }=1.56 \mathrm{~mm} \\
\text { Tan } \delta=0.02\end{array}$ \\
\hline Conductor & Gold \\
\hline
\end{tabular}

ematical description of the radiating tapers, length and width of the transition region and the ground plane patterning. Figure 4.5 labels these critical dimensions.

The first step in the design process is to design the microstrip feed for a $Z_{0}=50 \Omega$ characteristic impedance. If $W_{m s}$ represents the width of the microstrip line and $h$ represents the height of the dielectric substrate, then the required ratio is [13]:

$$
\frac{W_{m s}}{h}=\frac{8 e^{A}}{e^{2 A}-2}
$$

where, 


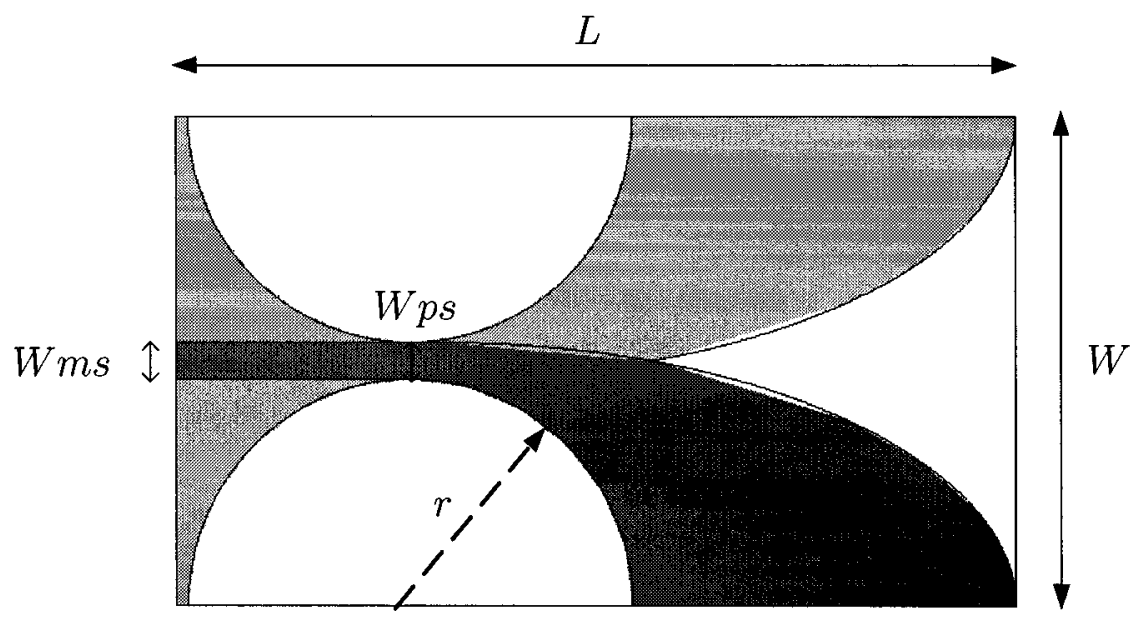

Figure 4.5: AVA schematic

$$
\mathrm{A}=\frac{Z_{0}}{60} \sqrt{\frac{\varepsilon_{r}+1}{2}}+\frac{\varepsilon_{r}-1}{\varepsilon_{r}+1}\left(0.23+\frac{0.11}{\varepsilon_{r}}\right)
$$

The variable substitutions were made based on the material information provided in Table 4.1, resulting in a microstrip width of $3.0 \mathrm{~mm}$. The ground plane slowly tapers along the AVA to transform the microstrip structure to a paired strip transmission line. The equation of this taper is circular with a radius calculated from:

$$
r=1 / 2\left(W-W_{p s}\right)
$$

The center of the circle, $c_{\text {circle }}$, is made to be on the edge of the substrate and a distance defined by Equation 4.3 away from the feed point:

$$
c_{\text {circle }}=\frac{W_{\text {sub }}}{2.0}-0.5 \quad[m m]
$$

Using [45], the width of the paired strip line to maintain $Z_{0}=50 \Omega$ is:

$$
Z_{0}=\frac{\eta_{0}}{\sqrt{\varepsilon_{r}}}\left\{\frac{W_{p s}}{h}+\frac{1.0}{\pi} \ln 4+\frac{\varepsilon_{r}+1.0}{2 \pi \varepsilon_{r}} \ln (a)+b\right\}
$$

where, 
$W_{p s}=$ width of the paired line, $\mathrm{h}=$ thickness of the dielectric substrate, $a=\frac{\pi e\left(W_{p s} / h+0.94\right)}{2.0}$, $b=\frac{\varepsilon_{r}-1.0}{2 \pi \varepsilon_{r}^{2}} \ln \frac{e \pi^{2}}{16.0}, \eta_{0}=377 \Omega$

This resulted in a paired strip width, $W_{p s}$, of $2.95 \mathrm{~mm}$. For simplicity, the value was taken to be $3.0 \mathrm{~mm}$. The paired strip width deviates more from the width of a simple microstrip line when the ratio of the microstrip width to substrate thickness is larger. An empirical analysis established the optimum length of the paired strip to be $1 \mathrm{~mm}$. At the end of the transition region, the paired strip metallization on either side of the substrate widens in opposite directions to become the radiating section of the antenna. The shape of this flare is critical to the performance of the AVA and should be as smooth as possible to improve high frequency performance. The interior of the flare is circular in shape and has the same radius defined by Equation 4.2. The exterior edge of the flare is elliptical in shape and is defined by Equations 4.5 and 4.6. The elliptical equations were developed to improve low frequency performance by maximizing the width of the slot aperture.

$$
\begin{gathered}
r_{\text {minor }}=r=1 / 2\left(W+W_{p s}\right) \\
\text { Ratio }=\frac{2\left(L-c_{\text {ellipse }}\right)}{W+W_{p s}} \quad[\mathrm{~mm}]
\end{gathered}
$$

Where, $c_{\text {ellipse }}$ is the location of the center of the ellipse along the edge of the AVA and after optimizations was selected to be $22 \mathrm{~mm}$ away from the feed plane. $\mathrm{L}$ and $\mathrm{W}$ are the length and width of the AVA, respectively. The length of the Vivaldi antenna is approximately one wavelength at the lowest frequency of operation, while the width is approximately half of one wavelength. Here, the lowest frequency of operation is $4 \mathrm{GHz}$, which corresponds 
to a length and width of $75 \mathrm{~mm}$ and $37.5 \mathrm{~mm}$, respectively. After optimizations, the final dimensions of the AVA were found to be $70 \mathrm{~mm}$ in length and $40 \mathrm{~mm}$ in width. Table 4.2 lists all the dimensions of the AVA calculated from equations 4.1 to 4.6.

Table 4.2: FR-4 AVA design parameters

\begin{tabular}{|c|c|c|c|c|c|c|}
\hline$W_{m s}$ & $W_{p s}$ & $c_{\text {circle }}$ & $\mathrm{r}$ & $r_{\text {minor }}$ & $c_{\text {ellipse }}$ & Ratio \\
\hline $3.0 \mathrm{~mm}$ & $3.0 \mathrm{~mm}$ & $19.5 \mathrm{~mm}$ & $18.5 \mathrm{~mm}$ & $18.5 \mathrm{~mm}$ & $22 \mathrm{~mm}$ & $2.2325 \mathrm{~mm}$ \\
\hline
\end{tabular}

The design of the LTCC based AVA follows the same procedure and uses the same equations as given for the FR-4 based AVA. However, only a $25 \mathrm{~mm} \times 50 \mathrm{~mm}$ rectangle was allocated on the LTCC substrate for the Vivaldi antenna. To meet the dimensional requirements, the AVA was selected to operate in the upper frequency band as defined by the DS-UWB group. The upper band occupies the spectrum from $6.2 \mathrm{GHz}-9.7 \mathrm{GHz}$. The complete design specifications are provided in Table 4.3. Because of the shortened Table 4.3: LTCC AVA specifications

\begin{tabular}{|c|c|}
\hline Bandwidth & $6.2 \mathrm{GHz}-9.7 \mathrm{GHz}$ \\
\hline Dimensions & $50 \mathrm{~mm} \times 25 \mathrm{~mm} \times 1.188 \mathrm{~mm}$ (12 layers) \\
\hline Substrate & FerroA6-S \\
& $\varepsilon_{r}=5.9$ \\
& Tan $\delta=0.0007$ \\
\hline Conductor & Silver \\
\hline
\end{tabular}

substrate length compared to the FR-4 AVA, the ellipse center, $c_{c e n t e r}$, was reduced from $22 \mathrm{~mm}$ to $15 \mathrm{~mm}$. Table 4.4 lists all the dimensions of the AVA calculated from equations 4.1 to 4.6 . 
Table 4.4: LTCC AVA design parameters

\begin{tabular}{|c|c|c|c|c|c|c|}
\hline$W_{m s}$ & $W_{p s}$ & $c_{\text {circle }}$ & $\mathrm{r}$ & $r_{\text {minor }}$ & $c_{\text {ellipse }}$ & Ratio \\
\hline $1.8 \mathrm{~mm}$ & $1.92 \mathrm{~mm}$ & $12 \mathrm{~mm}$ & $11.6 \mathrm{~mm}$ & $11.6 \mathrm{~mm}$ & $15 \mathrm{~mm}$ & $2.612 \mathrm{~mm}$ \\
\hline
\end{tabular}

\subsubsection{FDTD Source Excitation}

A broad band source excitation of the form discussed in Chapter 3, but repeated in Equation 4.7 for convenience, was used in all the FDTD simulations.

$$
\left.E_{z}\right|_{i, j, k} ^{n}=E_{0} e^{-\left(\frac{t-t_{0}}{T}\right)^{2}} \quad t_{0}=n_{0} \Delta t
$$

This pulse was designed to contain a relatively high amount of spectral energy up to $14 \mathrm{GHz}$. The Gaussian half-width was set to $\mathrm{T}=15 \mathrm{ps}$ and the time delay $t_{0}$ was set to be 3T, as is typically chosen [33]. The reference plane at which the E-field is sampled was chosen to be 5 cells away from the microstrip feed point. The simulations were performed for at least 8000 time steps to ensure a good frequency spectrum resolution.

\subsubsection{FR-4 based Antipodal Vivaldi Simulation}

The configuration of the AVA uses arcs and elliptically tapered geometry making it a challenging structure to model. Since in FDTD cubes are used to approximate smooth curves, a "staircase" effect will be created along the edges. However, this effect can be minimized by choosing small enough space increments. Table 4.5 shows the FDTD parameters used in the simulation of the AVA. $\Delta z$ is chosen so that three Yee cells exactly match the thickness of the substrate. $\Delta x$ and $\Delta y$ are chosen to exactly fit the width of the microstrip feed and the length of the AVA, respectively. Furthermore, this discretization satisfies the numerical dispersion criteria discussed in Chapter 3 and gives a reasonable 
Table 4.5: FR-4 based AVA FDTD analysis parameters

\begin{tabular}{|c|c|}
\hline Increment & Value $(\mathrm{mm})$ \\
\hline$\Delta x$ & 0.500 \\
\hline$\Delta y$ & 0.500 \\
\hline$\Delta z$ & 0.520 \\
\hline
\end{tabular}

approximation of the curved surface. The total mesh dimensions are $120 \times 160 \times 44$ in the $\hat{x}, \hat{y}$ and $\hat{z}$ directions, respectively.

Figure 4.6 shows the simulated time domain response and a comparison of the $\left|S_{11}\right|$ plots generated by HFSS and FDTD for the AVA constructed on an FR-4 substrate. The


Figure 4.6: Simulated FR-4 AVA return loss

plot indicates a broad impedance bandwidth of over $6 \mathrm{GHz}$, from $4 \mathrm{GHz}$ to $10 \mathrm{GHz}$. The agreement between the HFSS and FDTD results in this plot are good. From other time domain simulations, not shown here, the incident Gaussian pulse was seen to travel down the microstrip until it is partially reflected at the transition region. Very little reflected 
energy travels back along the microstrip, indicating a broad impedance bandwidth. The current is primarily confined to the microstrip feed and the edge of the radiating flares until it dissipates to almost zero at the end of the flares.

Two principle plane pattern cuts at $4 \mathrm{GHz}$ and $8 \mathrm{GHz}$ are made. The substrate lies in the $\mathrm{x}-\mathrm{y}$ plane with the nominal direction of propagation in the positive $\mathrm{x}$ direction. As shown in Figure 4.7, the slot is oriented along the y-axis for these plots. The simulated

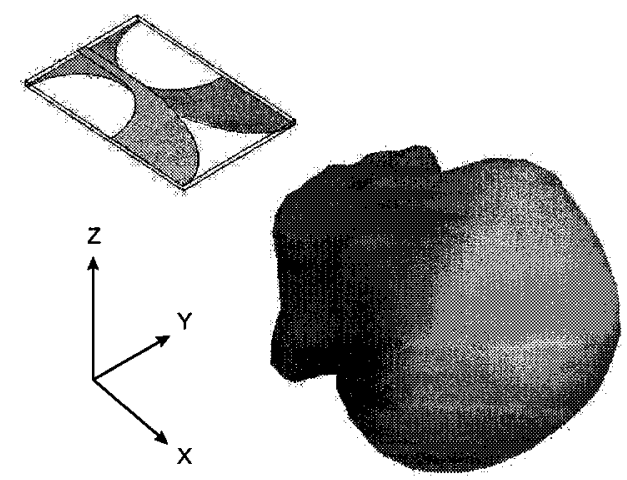

Figure 4.7: Simulated FR-4 based AVA 3-dimensional radiation pattern

radiation characteristics of the AVA are displayed in Figures 4.8 to 4.11.

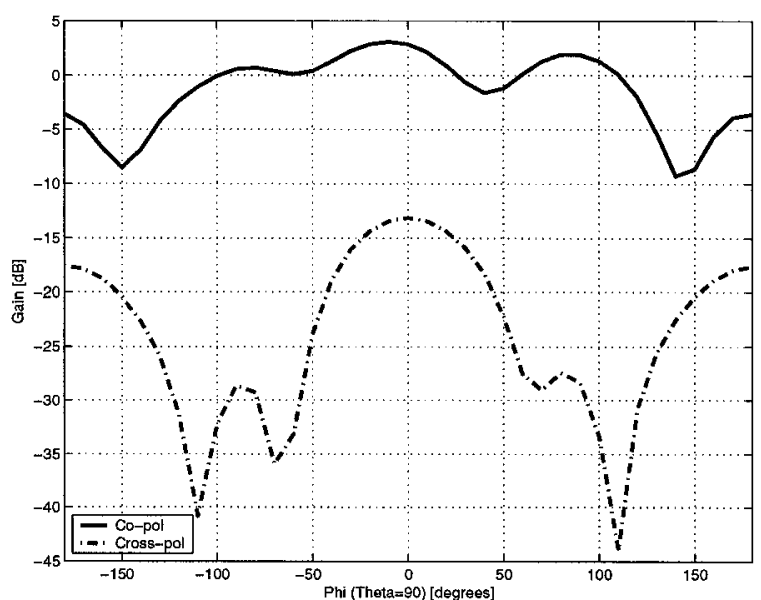

Figure 4.8: Simulated FR-4 AVA $4 \mathrm{GHz}$ $\mathrm{XY}$-plane



Figure 4.9: Simulated FR-4 AVA $4 \mathrm{GHz}$ $\mathrm{XZ}$-plane 


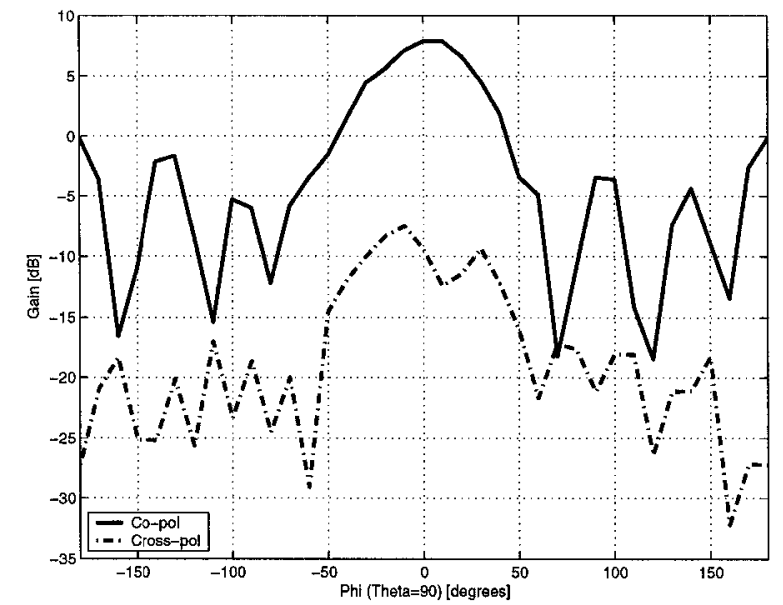

Figure 4.10: Simulated FR-4 AVA $8 \mathrm{GHz}$ XY-plane



Figure 4.11: Simulated FR-4 AVA $8 \mathrm{GHz}$ $\mathrm{XZ}$-pane

The results show a directional radiation pattern with a maximum gain at $4 \mathrm{GHz}$ of $3 \mathrm{~dB}$ at boresight, rising to $7.9 \mathrm{~dB}$ at $8 \mathrm{GHz}$. The half-power beamwidth (HPBW) is very broad at $4 \mathrm{GHz}$ in both the xy and xz-plane being 125 degrees and 120 degrees, respectively. At $8 \mathrm{GHz}$ the HPBW shrinks significantly to 65 degrees and 60 degrees in the xy and xz-plane, respectively. The curved ends of the antenna cause a creeping wave to exist that contributes to a relatively high back lobe at 180 degrees from boresight [31]. The front to back ratio (FBR) is calculated as the difference in gain at boresight and of the back lobe. The FBR at $4 \mathrm{GHz}$ and $8 \mathrm{GHz}$ is $6.7 \mathrm{~dB}$ and $7.9 \mathrm{~dB}$, respectively. In all cases the cross-polarization levels remain mostly $15 \mathrm{~dB}$ below the co-polarized values. These

Table 4.6: Simulated FR-4 AVA radiation pattern properties

\begin{tabular}{|c|c|c|c|c|c|}
\hline Frequency & xy HPBW & xz HPBW & Gain & FBR & Cross-pol level \\
\hline $4 \mathrm{GHz}$ & $125 \mathrm{deg}$ & $120 \mathrm{deg}$ & $3.09 \mathrm{~dB}$ & $6.69 \mathrm{~dB}$ & $-15 \mathrm{~dB}$ \\
\hline $8 \mathrm{GHz}$ & $65 \mathrm{deg}$ & $60 \mathrm{deg}$ & $7.85 \mathrm{~dB}$ & $7.97 \mathrm{~dB}$ & $-17 \mathrm{~dB}$ \\
\hline
\end{tabular}

radiation characteristics are consistent with previously published AVA antennas. Table 
4.6 summarizes the simulated data.

For the near field probe simulation, the sample location is chosen to be at a grid location that corresponds to the direction of maximum radiation. Sampling the co-polarized, $E_{x}$, component of the electric field results in the plot of Figure 4.12.

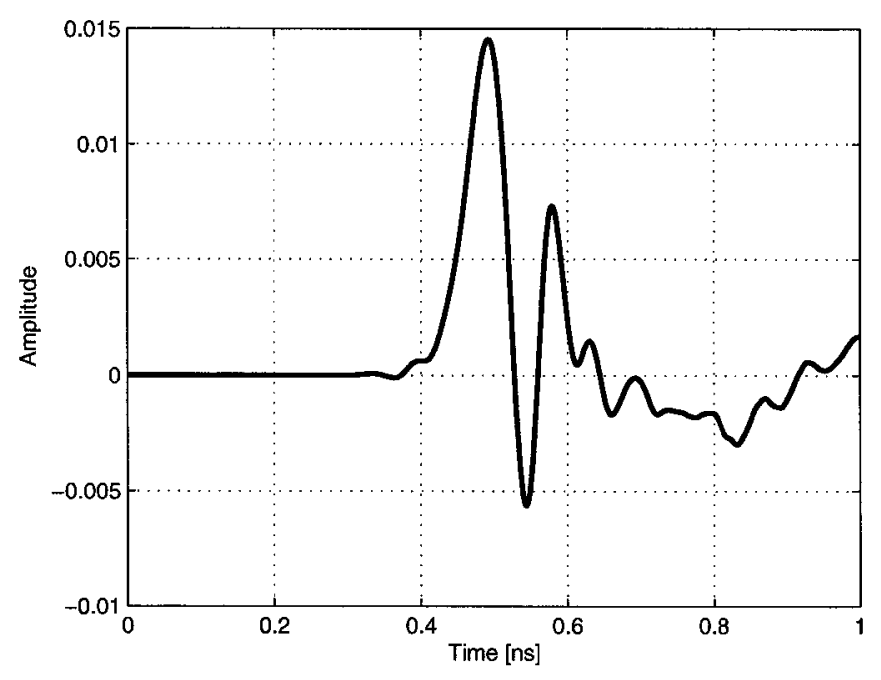

Figure 4.12: Simulated near field probe result for FR-4 AVA

As predicted in [46], the waveform of Figure 4.12 resembles the first derivative of the Gaussian pulse applied to the antenna input. Nevertheless this result is significantly better than that achieved with the patch antenna of Chapter 3 and serves as an indication of good time domain performance for the FR-4 AVA.

\subsubsection{LTCC-based antipodal Vivaldi simulation}

As with the FR4-based Vivaldi antenna, the configuration of the LTCC-based AVA presents a challenging structure to model. Accordingly, Table 4.7 shows the FDTD parameters used in the simulation of the AVA. $\Delta z$ is chosen so that three Yee cells exactly 
Table 4.7: LTCC based AVA FDTD analysis parameters

\begin{tabular}{|c|c|}
\hline Increment & Value $(\mathrm{mm})$ \\
\hline$\Delta x$ & 0.450 \\
\hline$\Delta y$ & 0.500 \\
\hline$\Delta z$ & 0.396 \\
\hline
\end{tabular}

match the thickness of the substrate. $\Delta x$ and $\Delta y$ are chosen to match as closely as possible the width of the microstrip feed and the length of the AVA, respectively. The total mesh dimensions are $95 \times 120 \times 44$ in the $\hat{x}, \hat{y}$ and $\hat{z}$ directions, respectively.

Figure 4.13 shows the simulated time domain response and a comparison of the $\left|S_{11}\right|$ plots generated by HFSS and FDTD for the AVA constructed on an LTCC substrate. The plot indicates a broad impedance bandwidth from $6.2 \mathrm{GHz}$ to $10 \mathrm{GHz}$. The simulated
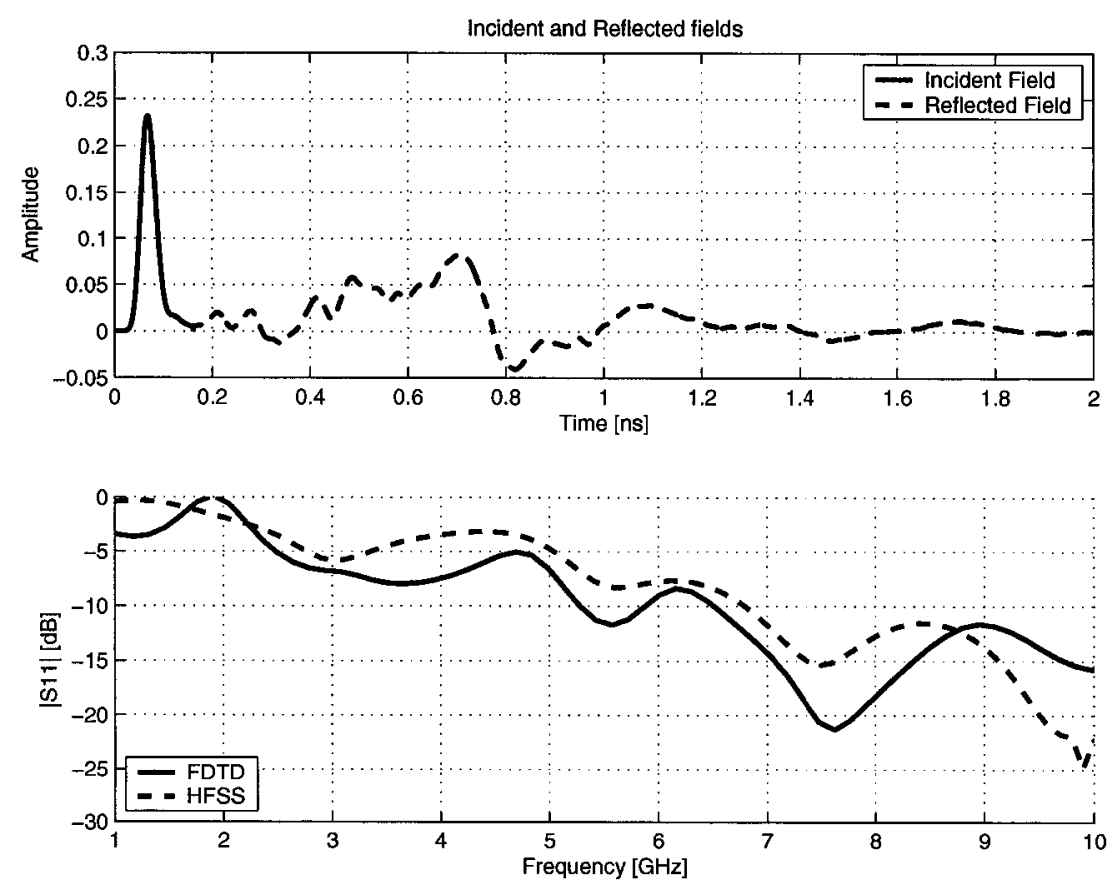

Figure 4.13: Simulated LTCC AVA return loss

radiation characteristics of the AVA are displayed in Figures 4.16 to 4.17. The substrate 
lies in the $\mathrm{x}-\mathrm{y}$ plane with the nominal direction of propagation in the positive $\mathrm{x}$ direction. The slot is oriented along the y-axis for these plots. Two different pattern cuts at $6 \mathrm{GHz}$ and $8 \mathrm{GHz}$ are shown. The results show a directional pattern with a maximum gain

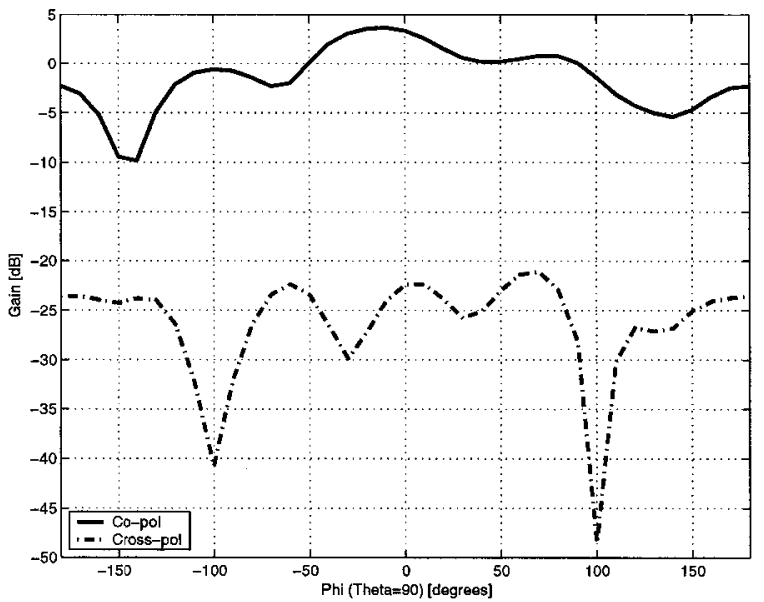

Figure 4.14: Simulated LTCC AVA $6 \mathrm{GHz}$ $\mathrm{XY}$-plane

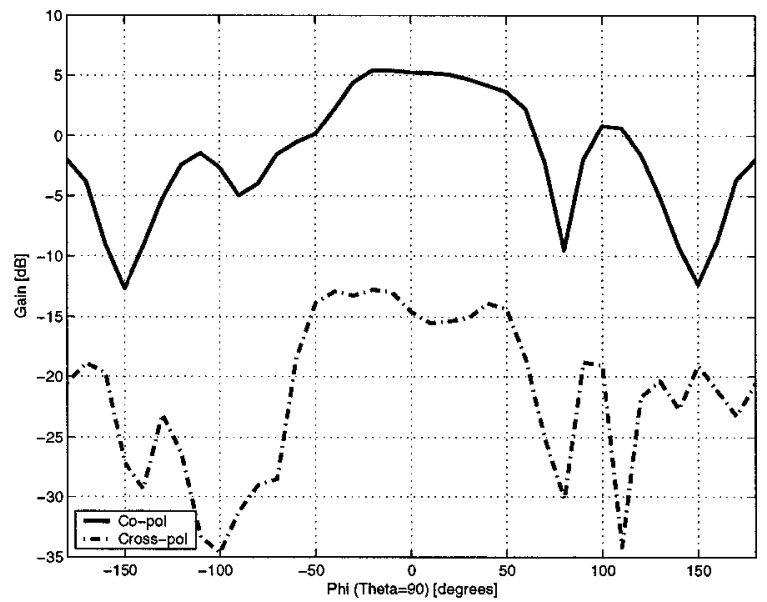

Figure 4.16: Simulated LTCC AVA $8 \mathrm{GHz}$ $\mathrm{XY}$-plane

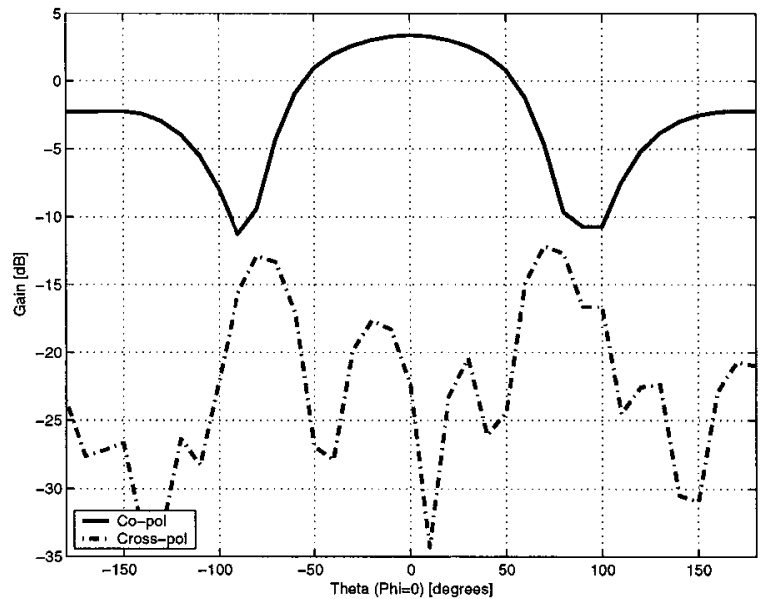

Figure 4.15: Simulated LTCC AVA $6 \mathrm{GHz}$ XZ-plane

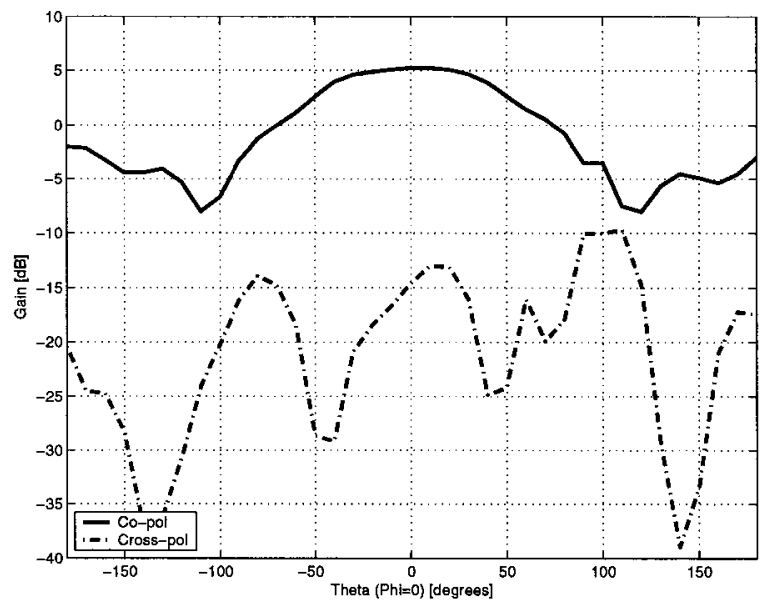

Figure 4.17: Simulated LTCC AVA $8 \mathrm{GHz}$ XZ-plane

at $6 \mathrm{GHz}$ of $3.69 \mathrm{~dB}$ at boresight. Gain increases to $5.4 \mathrm{~dB}$ at $8 \mathrm{GHz}$. The HPBW is very broad at $6 \mathrm{GHz}$ in both the xy and xz-plane being 140 degrees and 105 degrees, respectively. At $8 \mathrm{GHz}$ the $\mathrm{HPBW}$ shrinks slightly to 105 degrees and 100 degrees in 
the xy and xz-plane, respectively. The FBR at $6 \mathrm{GHz}$ and $8 \mathrm{GHz}$ is $5.97 \mathrm{~dB}$ and $7.4 \mathrm{~dB}$, respectively. In all cases the cross-polarization levels remain $20 \mathrm{~dB}$ below the co-polarized levels. Table 4.8 summarizes the simulated data.

Table 4.8: Simulated LTCC AVA radiation pattern properties

\begin{tabular}{|c|c|c|c|c|c|}
\hline Frequency & xy HPBW & xz HPBW & Gain & FBR & Cross-pol level \\
\hline $6 \mathrm{GHz}$ & $180 \mathrm{deg}$ & $105 \mathrm{deg}$ & $3.69 \mathrm{~dB}$ & $5.97 \mathrm{~dB}$ & $-25 \mathrm{~dB}$ \\
\hline $8 \mathrm{GHz}$ & $105 \mathrm{deg}$ & $100 \mathrm{deg}$ & $5.4 \mathrm{~dB}$ & $7.4 \mathrm{~dB}$ & $-20 \mathrm{~dB}$ \\
\hline
\end{tabular}

For the near field probe simulation, the sample location is chosen to be at a grid location that corresponds to the direction of maximum radiation. Sampling the co-polarized, $E_{x}$, component of the electric field results in the plot of Figure 4.18. As with the FR4

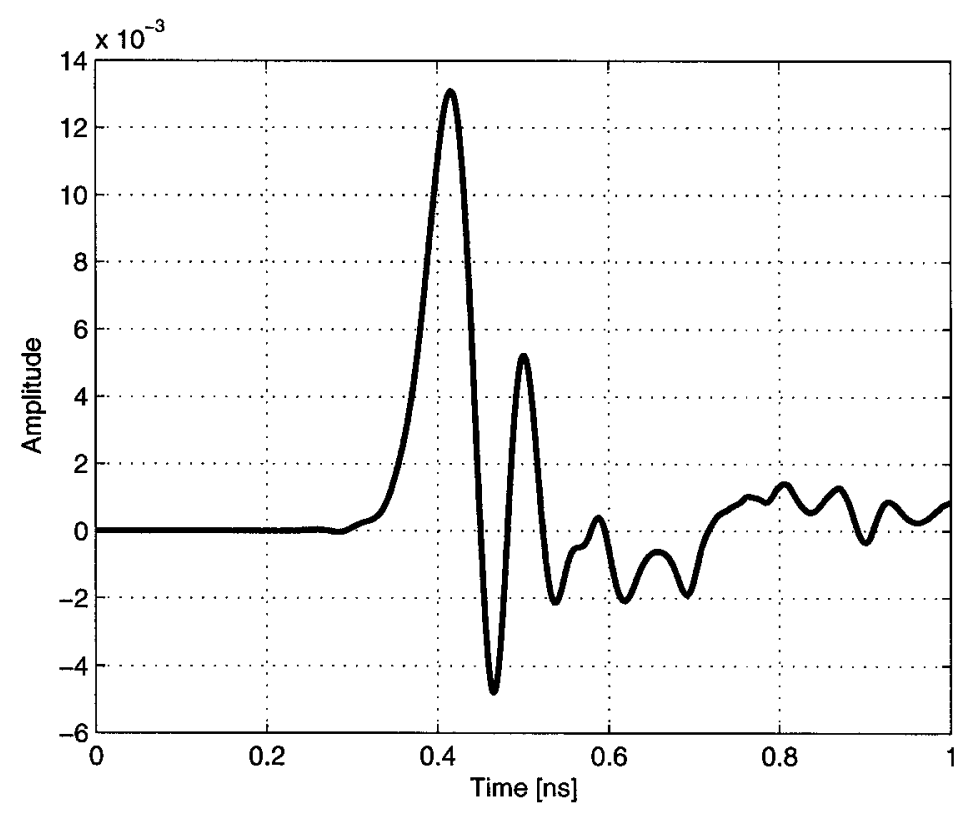

Figure 4.18: Simulated near field probe result for LTCC AVA

AVA, the waveform resembles the first derivative of the Gaussian pulse applied to the antenna input but to a lesser extent. In this case the oscillations at the tail of the main 
pulse degrade more quickly. Thus, this result also serves as an indication of good time domain performance for the LTCC AVA.

\subsection{Balanced Antipodal Vivaldi Antenna Design and Simulation}

As expected and confirmed in some of the simulation results in the previous sections, the AVA suffers from poor cross-polarization performance at high frequencies due to the skew in the electric fields across the flared slot aperture. To overcome this high cross polarization, J. Langley et. al [43] have added a further layer of metallization to form a B-AVA. Figure 4.19 shows a cross-sectional view of the AVA and B-AVA. The skew is eliminated in the B-AVA by the cancelation of the perpendicular electric field components, leaving the resultant E-field $(R)$ oriented vertically. The third metal layer is identical to

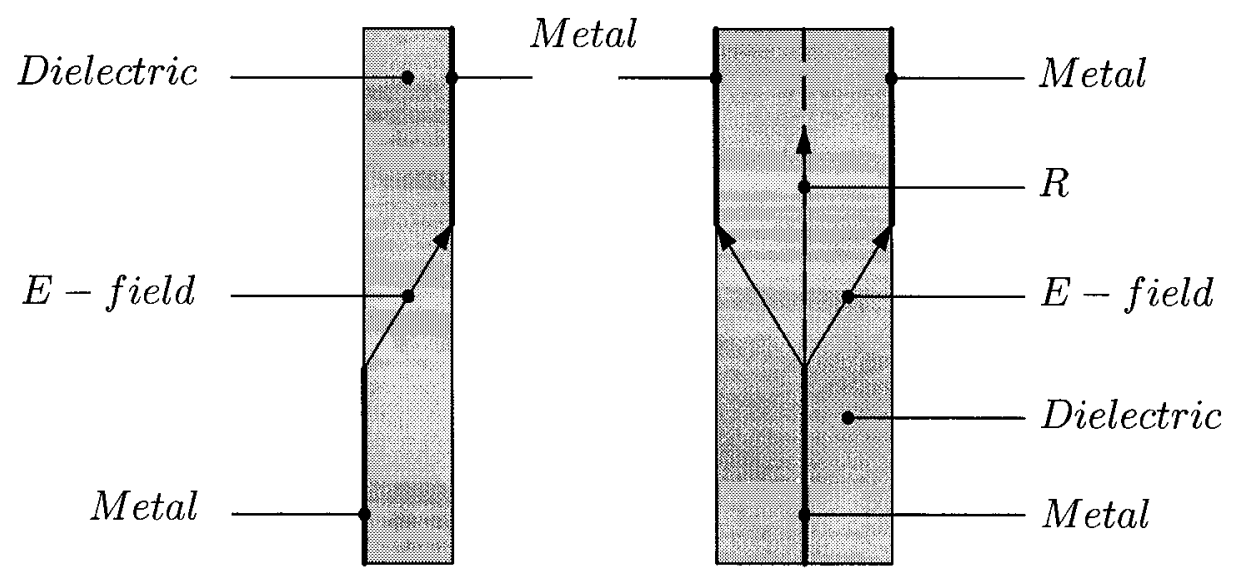

Figure 4.19: Cross-section of AVA and B-AVA

the bottom layer (ground) and is spaced an equal distance from the stripline feed as the bottom layer. The two ground layers are then connected with vias near the lower and upper left portions of the schematic. Figure 4.20 shows the variables used in the design of 
the B-AVA. An LTCC based B-AVA was designed to the same specifications as the LTCC

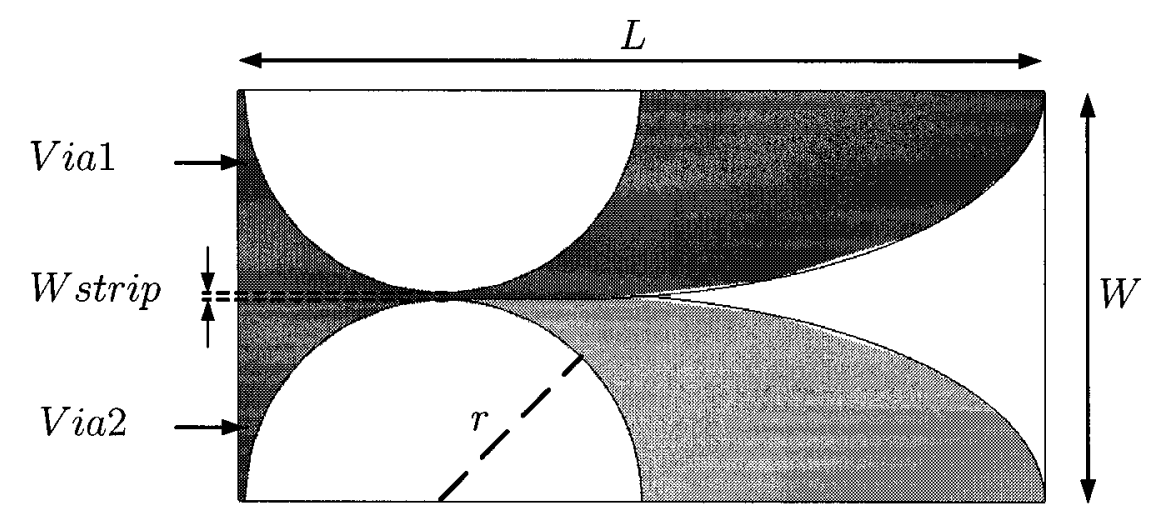

Figure 4.20: B-AVA schematic

based AVA. However, it was not fabricated because of the extra connector complexity associated with the feed method. The tri-plate structure of the B-AVA forms a stripline feed, which is the most important difference from the AVA. The width of the stripline was designed for a $Z_{0}=50 \Omega$ characteristic impedance. If $W_{\text {strip }}$ represents the width of the stripline and $h$ represents the height of the substrate, then the required ratio is [45]:

$$
\frac{W_{\text {strip }}}{h}=0.85-\sqrt{0.6-x}
$$

where, $x=\frac{30 \pi}{\sqrt{\varepsilon_{r}} Z_{0}-0.441}$

This resulted in a stripline width of $0.4 \mathrm{~mm}$, significantly narrower than the $1.8 \mathrm{~mm}$ width of the microstrip feedline. As with the AVA, Equations 4.2, 4.5 and 4.6 were used to create the circular and elliptical tapers in the balanced ground layers. Here, the variable $c_{\text {ellipse }}$ was given a value of $20 \mathrm{~mm}$. To simplify testing, a microstrip to stripline transition could be incorporated by creating a cavity in the upper half of the substrate and exposing the feed line. Table 4.9 lists all the dimensions of the B-AVA calculated from equations $4.2,4.3,4.5,4.6$ and 4.8 . 
Table 4.9: LTCC B-AVA design parameters

\begin{tabular}{|c|c|c|c|c|c|}
\hline$W_{\text {strip }}$ & $c_{\text {circle }}$ & $\mathrm{r}$ & $r_{\text {minor }}$ & $c_{\text {ellipse }}$ & Ratio \\
\hline $0.4 \mathrm{~mm}$ & $12 \mathrm{~mm}$ & $12.75 \mathrm{~mm}$ & $12.75 \mathrm{~mm}$ & $20 \mathrm{~mm}$ & $2.362 \mathrm{~mm}$ \\
\hline
\end{tabular}

\subsubsection{LTCC-based Balanced Antipodal Vivaldi Simulation}

Because of the extra modeling challenge presented by the stripline feed, the B-AVA was exclusively simulated in HFSS. Figure 4.21 shows the simulated $\left|S_{11}\right|$ plot for the B-AVA. The plot indicates an impedance bandwidth from $6.5 \mathrm{GHz}$ to $10 \mathrm{GHz}$, with a small peak

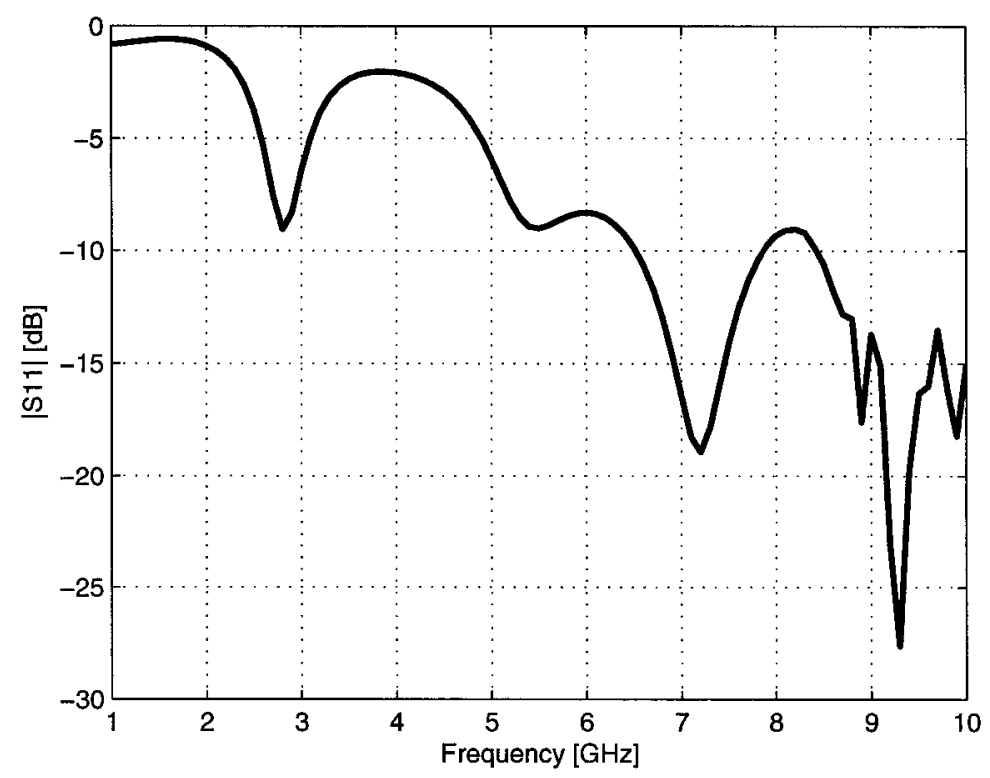

Figure 4.21: Simulated B-AVA return loss

above $-10 \mathrm{~dB}$ at $8.2 \mathrm{GHz}$.

The radiation characteristics of the B-AVA are displayed in Figures 4.22 to 4.25. The substrate lies in the $\mathrm{x}-\mathrm{y}$ plane with the nominal direction of propagation in the positive $\mathrm{x}$ direction. The slot is oriented along the y-axis for these plots. Two different pattern cuts at $6 \mathrm{GHz}$ and $8 \mathrm{GHz}$ are shown. 


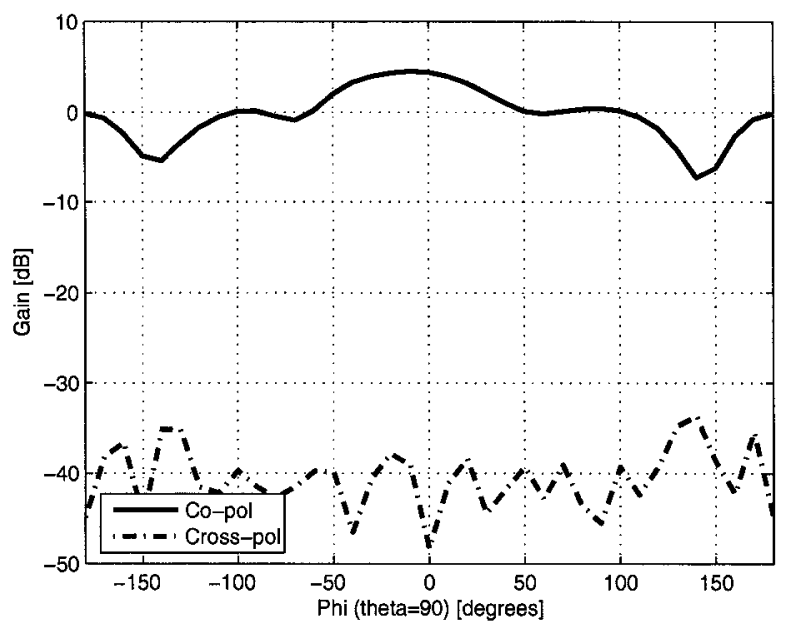

Figure 4.22: Simulated LTCC B-AVA $6 \mathrm{GHz}$ XY-plane

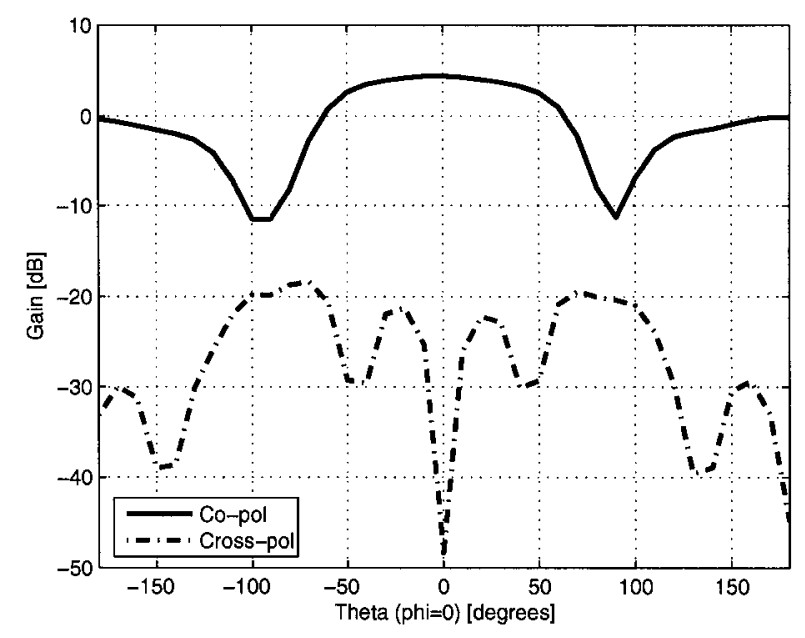

Figure 4.23: Simulated LTCC B-AVA $6 \mathrm{GHz}$ XZ-plane

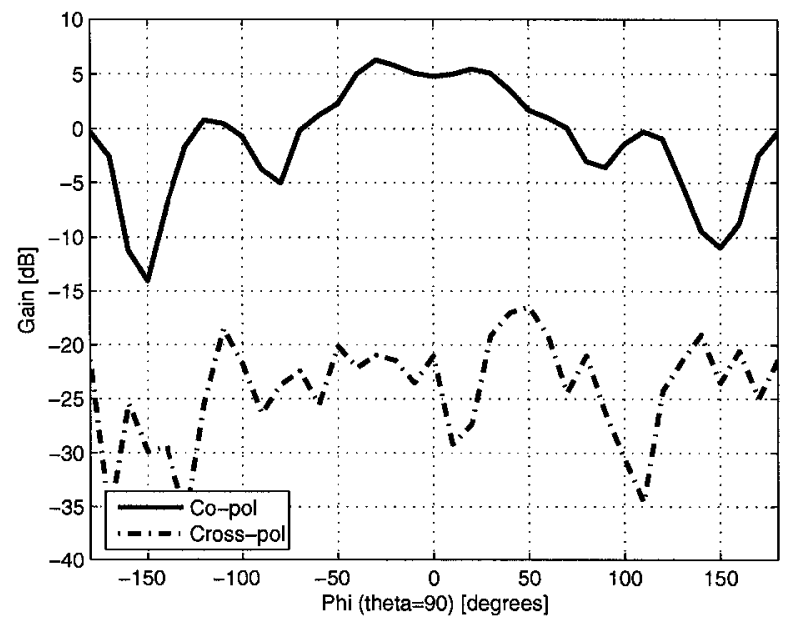

Figure 4.24: Simulated LTCC B-AVA $8 \mathrm{GHz} \mathrm{XY}$-plane



Figure 4.25: Simulated LTCC B-AVA $8 \mathrm{GHz}$ XZ-plane 
The results show an almost omni-directional radiation pattern at $6 \mathrm{GHz}$ in the $\mathrm{xy}-$ plane with a maximum gain of $4.4 \mathrm{~dB}$. At $8 \mathrm{GHz}$ the gain increases to $6.3 \mathrm{~dB}$ at 30 degrees off boresight, which is $1 \mathrm{~dB}$ higher than the maximum gain achieved by the LTCC AVA. The HPBW is very broad at $6 \mathrm{GHz}$ in both the xy and xz-plane being 95 degrees and 105 degrees, respectively. At $8 \mathrm{GHz}$ the HPBW increases slightly to 120 degrees in both the xy and xz-plane. The FBR at $6 \mathrm{GHz}$ and $8 \mathrm{GHz}$ is $4.57 \mathrm{~dB}$ and $5.03 \mathrm{~dB}$, respectively. In all cases the cross-polarization levels remain $25 \mathrm{~dB}$ below the co-polarization levels and are $5 \mathrm{~dB}$ to $10 \mathrm{~dB}$ lower than those of the LTCC AVA. This result confirms that the three layer B-AVA does have improved cross-polarization performance with respect to the two-layer AVA. Table 4.10 summarizes the simulated data.

Table 4.10: Simulated LTCC BAVA radiation pattern properties

\begin{tabular}{|c|c|c|c|c|c|}
\hline Frequency & xy HPBW & xz HPBW & Gain & FBR & Cross-pol level \\
\hline $6 \mathrm{GHz}$ & $95 \mathrm{deg}$ & $105 \mathrm{deg}$ & $4.44 \mathrm{~dB}$ & $4.57 \mathrm{~dB}$ & $-40 \mathrm{~dB}$ \\
\hline $8 \mathrm{GHz}$ & $120 \mathrm{deg}$ & $120 \mathrm{deg}$ & $6.27 \mathrm{~dB}$ & $5.03 \mathrm{~dB}$ & $-25 \mathrm{~dB}$ \\
\hline
\end{tabular}

\subsection{UWB Monopole Antenna Theory}

Classical dipole and monopole antennas are some of the most widely used antennas in the world today. However, they are inherently narrowband and thus unsuitable for UWB applications. However, recent work in [47] [48] and [49] has shown that by using a disc or square shaped monopole broadband and multi-band performance can be achieved. Here, a new triangular monopole that mimics the shape of a single-ended bow-tie antenna is proposed. The bow-tie antenna is inherently broadband and was first discussed in 1898 
by Oliver Lodge [50]. In the late 1950's Brown and Woodward [51] performed the first comprehensive investigation of the input impedance and radiation of this antenna. Figures 4.26 and 4.27 show balanced and single ended versions of the bow-tie antenna, respectively.

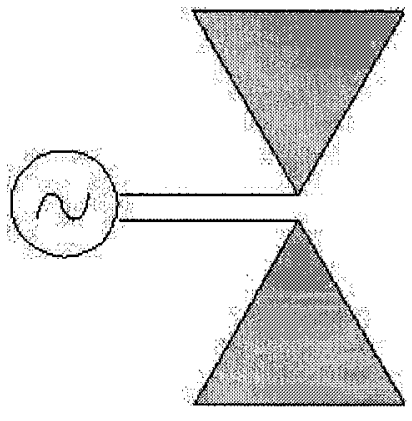

Figure 4.26: Bow-tie antenna

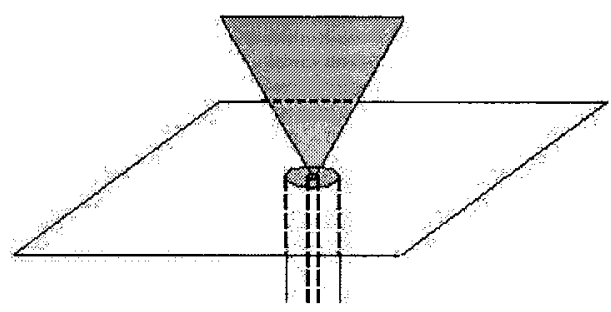

Figure 4.27: Triangular sheet antenna

Because of its finite size, the bow-tie is not frequency independent. It is a resonant type antenna with linear polarization. It has a bidirectional pattern with broad main beams perpendicular to the plane of the antenna. It has traditionally been used as a UHF TV antenna with a wire grid back plane to reduce the back lobe [14].

The triangular monopole antenna can be designed to be low cost and compact with much broader bandwidth performance than popular microstrip antennas. Microstrip antennas are limited to only a few percent bandwidth, while triangular monopole antennas can have a bandwidth of $88 \%$ [52].

Two different triangular monopole antennas were designed and fabricated using low cost FR-4 and low-loss LTCC substrates. Both antennas incorporate a novel feature to increase their bandwidth and efficiency. This feature acts as a capacitive load that reduces the input impedance variation with frequency. Both antennas are single-ended to avoid the need for a bandwidth limiting balun and to reduce their overall size. 


\subsection{Partial Ground Plane Triangular Monopole An- tenna Design and Simulation}

Recently, a simple antenna topology that incorporates a microstrip feed and partial ground plane has been used to design broadband [19] [20] or multi-band antennas [53]. This topology makes it possible to combine the antenna with integrated RF electronics and passive lumped components. Also, there is no need for a costly and performance limiting balun. The antenna presented here, called a partial ground plane triangular monopole (PGP-TM), is a two metal layer structure with a bow-tie shaped lower section with two adjacent triangular metal elements. One version of this antenna was constructed on a low-cost FR-4 substrate and another on a low loss LTCC substrate. Figure 4.28 shows the configuration of the proposed UWB antenna.

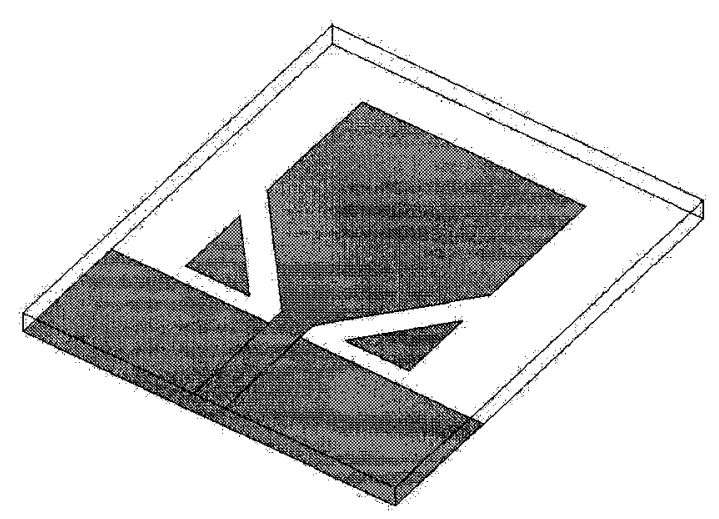

Figure 4.28: PGP-TM (angled view)

The PGP-TM was designed to operate in the lower frequency band as defined by the DS-UWB group. Table 4.11 displays the performance and material specifications of both substrate implementations.

The topology of the PGP-TM mimics a bow-tie shape to create a wide impedance 
Table 4.11: FR-4 based PGP-TM specifications

\begin{tabular}{|c|c|c|}
\hline & FR-4 PGP-TM & LTCC PGP-TM \\
\hline Bandwidth & $3.1 \mathrm{GHz}-4.85 \mathrm{GHz}$ & $3.1 \mathrm{GHz}-4.85 \mathrm{GHz}$ \\
\hline Substrate & FR-4 & Ferro A6S \\
& $\varepsilon_{r}=4.4$ & $\varepsilon_{r}=5.9$ \\
& Tan $\delta=0.02$ & Tan $\delta=0.0007$ \\
& height $=1.56 \mathrm{~mm}$ & height $=1.188 \mathrm{~mm}$ \\
\hline Conductor & Gold & Silver \\
\hline
\end{tabular}

bandwidth. The microstrip feed line diverges linearly at an angle of 40 degrees (from center) to make the bow-tie shaped section. The partial ground plane terminates when the top layer metallization begins to diverge to form the triangular monopole shaped radiating element. A rectangular metal area forms the rest of the main section of the antenna. Two novel parasitic elements on either side of the bow-tie section serve as capacitive loads. Capacitive loading reduces the input impedance variation with frequency of the antenna, while maintaining its efficiency. The capacitance can be adjusted by varying the separation between the parasitic elements and the main part of the antenna. This feature provides another important parameter that can be used to change the performance of the antenna. The effect of parasitic element separation was explored using HFSS and is shown in section 4.5.2.

For both substrate implementations of the PGP-TM, equation 4.1 was used to design the microstrip feed sections for a characteristic impedance of $50 \Omega$. Resulting in microstrip widths $\left(W_{m s}\right)$ of $3.0 \mathrm{~mm}$ and $1.8 \mathrm{~mm}$ for the FR-4 and LTCC implementations, respectively. A parametric analysis in HFSS was used to vary the other dimensions of 
the antennas. With similar impedance bandwidths, the LTCC-based PGP-TM is $14 \%$ shorter and $20 \%$ narrower than its FR-4 counterpart.

A diagram depicting the topology with dimensional information indicated is shown in Figure 4.29 The final optimized dimensions of the FR-4 and LTCC-based PGP-TM antennas are presented in Table 4.12. These final dimensions were used to model the antennas with the FDTD technique.

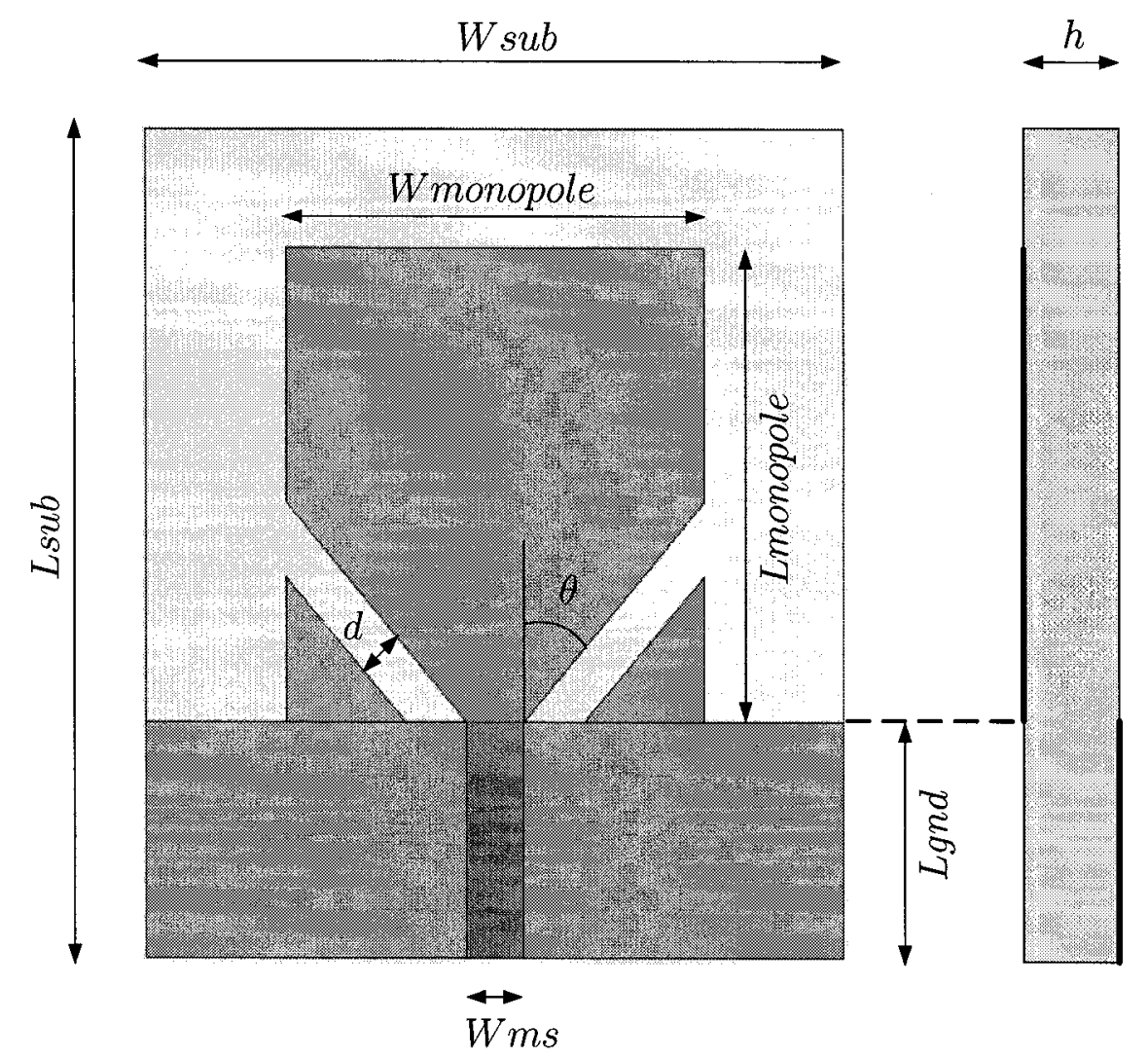

Figure 4.29: PGP-TM topology

Table 4.12: PGP-TM dimensions

\begin{tabular}{|c|c|c|c|c|c|c|c|c|}
\hline & $L_{\text {sub }}$ & $W_{\text {sub }}$ & $L_{\text {gnd }}$ & $L_{\text {monopole }}$ & $W_{\text {ms }}$ & $d$ & $W_{\text {monopole }}$ & $\theta$ \\
\hline FR-4 [mm] & 35 & 30 & 10 & 20 & 3.0 & 2.0 & 18 & $40 \mathrm{deg}$ \\
\hline LTCC [mm] & 30 & 25 & 7.5 & 20 & 1.8 & 2.0 & 18 & $40 \mathrm{deg}$ \\
\hline
\end{tabular}




\subsubsection{FR-4 Based Partial Ground Plane Triangular Monopole Simulation}

The configuration of the PGP-TM is simpler than the Vivaldi antenna, however a "staircase" approximation is still used on the angled edges of the antenna and especially on the parasitic elements. Table 4.14 shows the FDTD parameters used in the simulation of the FR-4 based PGP-TM. $\Delta z$ is chosen so that three Yee cells exactly match the thickness of

Table 4.13: FR-4 based PGP-TM FDTD analysis parameters

\begin{tabular}{|c|c|}
\hline Increment & Value $(\mathrm{mm})$ \\
\hline$\Delta x$ & 0.500 \\
\hline$\Delta y$ & 0.500 \\
\hline$\Delta z$ & 0.520 \\
\hline
\end{tabular}

the substrate. $\Delta x$ and $\Delta y$ are chosen to exactly fit the width of the microstrip feed and the length of the antenna, respectively. The total mesh dimensions are $100 \times 90 \times 44$ in the $\hat{x}, \hat{y}$ and $\hat{z}$ directions respectively.

Figure 4.30 shows the simulated time domain response of the FR4-based PGP-TM and a comparison of the $\left|S_{11}\right|$ plots generated by HFSS and FDTD.

The impedance bandwidth ranges from $2.7 \mathrm{GHz}$ to $7 \mathrm{GHz}$. The FDTD simulation results agree well with those generated by HFSS. However, the FDTD program shows a lower $\left|S_{11}\right|$ minimum at $4 \mathrm{GHz}$.

From other time domain simulations, not shown here, the incident Gaussian pulse was seen to travel down the microstrip until it is partially reflected at the point where the microstrip line begins to diverge. Very high electric field intensities are observed at 

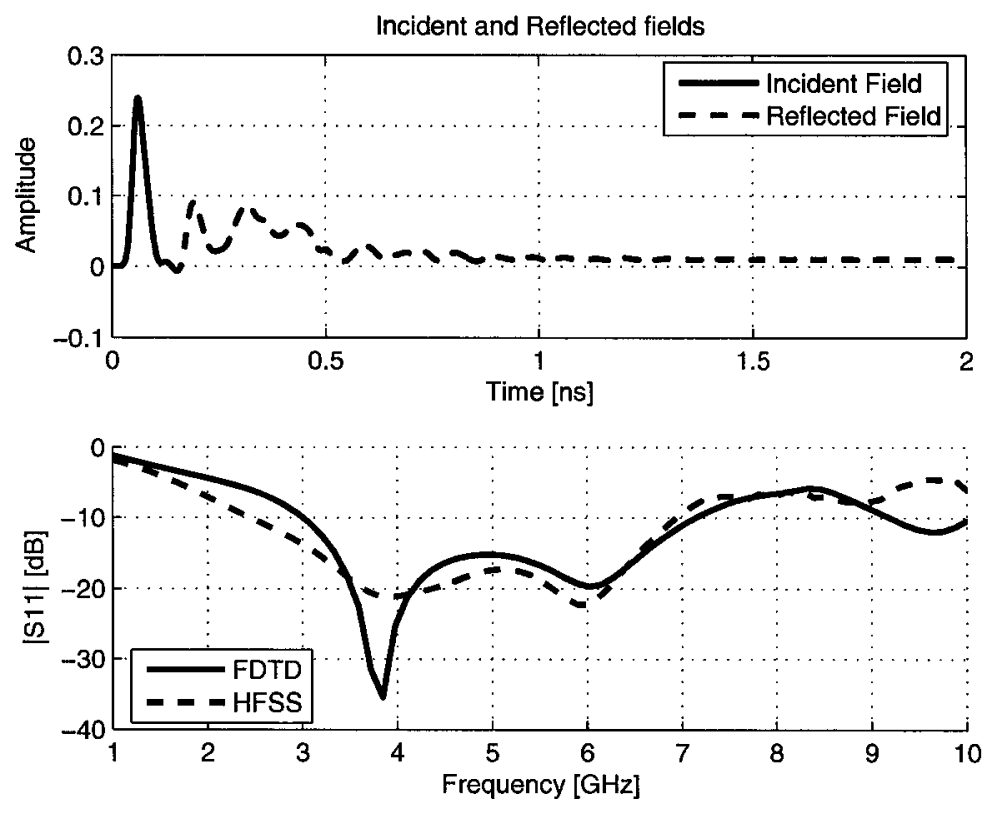

Figure 4.30: Simulated FR-4 PGP-TM return loss

the corners of the parasitic elements, confirming that they do effect antenna performance. Very little reflected energy travels back along the microstrip, indicating a broad impedance bandwidth. The current is primarily confined to the microstrip feed and the triangular edges of the antenna.

The 2-dimensional radiation characteristics at $4 \mathrm{GHz}$ and $6 \mathrm{GHZ}$ of the PGP-TM are displayed in Figures 4.31 to 4.34. The substrate lies in the $\mathrm{x}-\mathrm{y}$ plane with the microstrip feed line oriented along the x-axis. The results show a stable omni-directional pattern with a peak gain of $3.47 \mathrm{~dB}$ at $6 \mathrm{GHz}$. The cross-polarization levels are below $-20 \mathrm{~dB}$.

A recent study has shown that consistency of the radiated pulse shape is a critical issue in UWB antenna performance [54]. The study shows that even an antenna system exhibiting an isotropic radiation pattern at all frequencies does not imply that pulse fidelity is identical in all directions. An antenna with true omnidirectional performance 


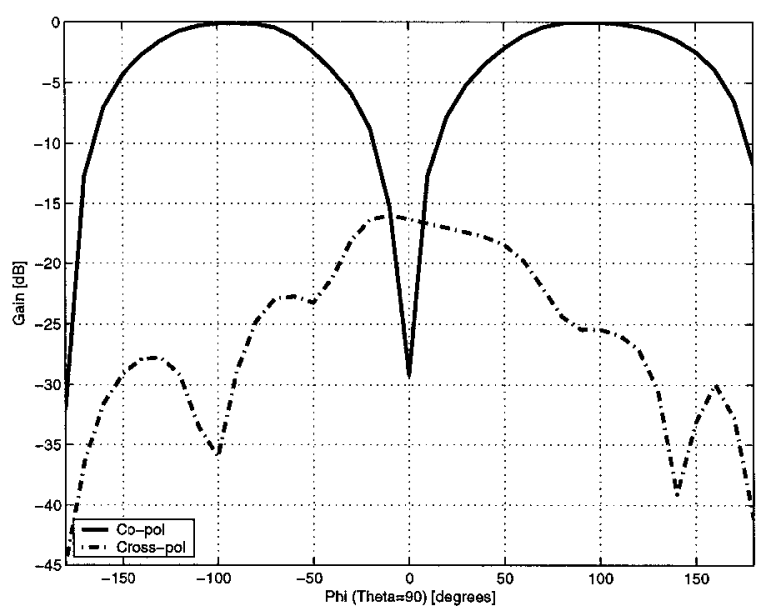

Figure 4.31: Simulated FR-4 PGP-TM $4 \mathrm{GHz}$ XY-plane

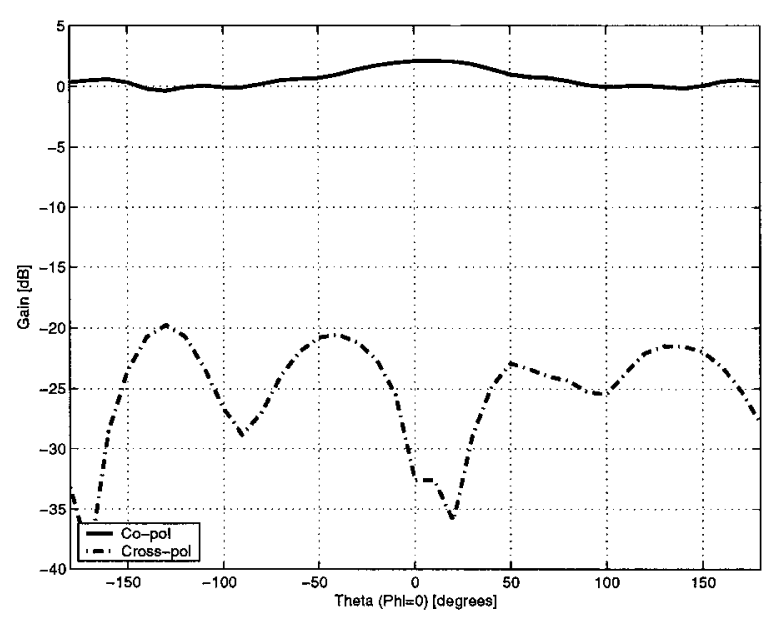

Figure 4.32: Simulated FR-4 PGP-TM $4 \mathrm{GHz}$ YZ-plane



Figure 4.33: Simulated FR-4 PGP-TM $6 \mathrm{GHz} X Y$-plane

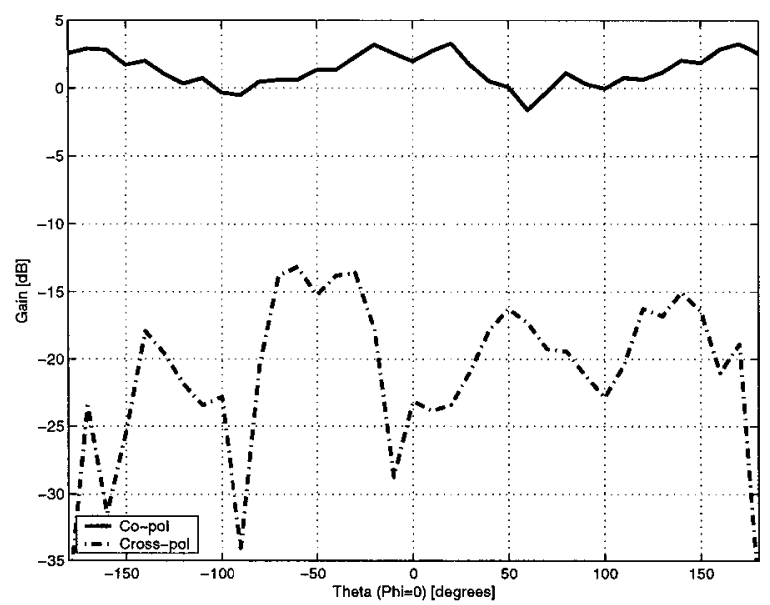

Figure 4.34: Simulated FR-4 PGP-TM $6 \mathrm{GHz}$ YZ-plane 
is said to have very good pulse fidelity.

To verify the pulse fidelity of the omnidirectional PGP-TM, three different sampling locations are selected and their results are compared. The near field probes are placed to the left, middle and right of the antenna and at a height above the plane of the antenna. Sampling the co-polarized, $E_{y}$, component of the electric field at these locations results in the plot of Figure 4.35 .

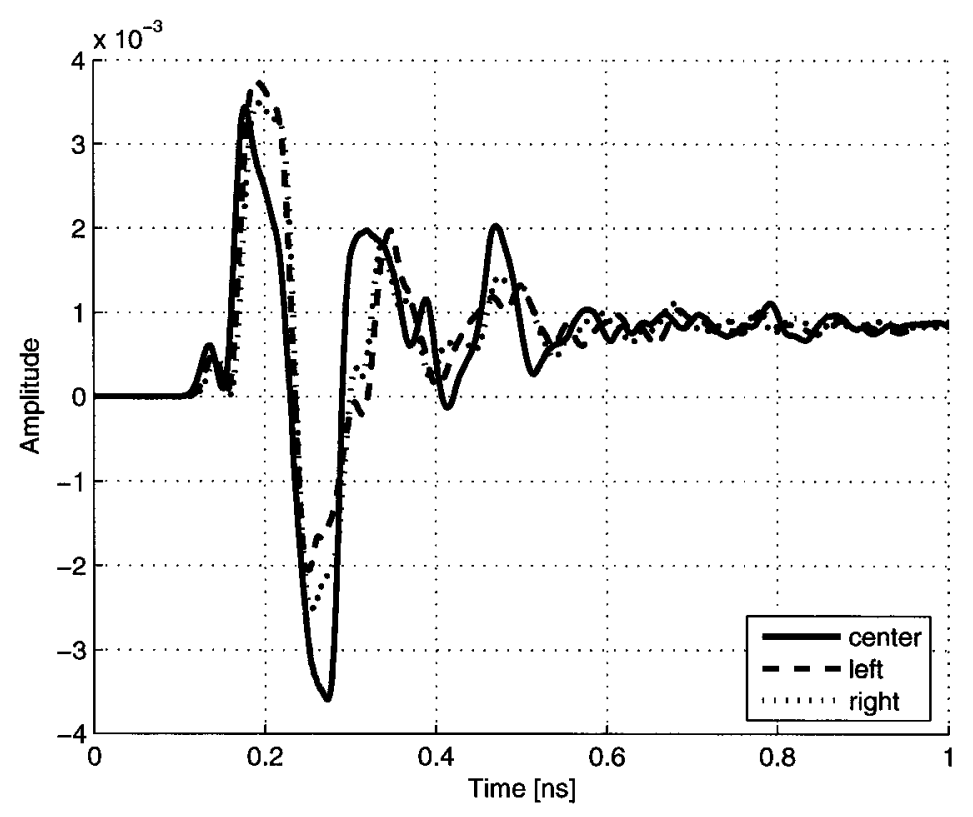

Figure 4.35: Simulated near field probe result for FR-4 PGP-TM

The close similarity in the waveforms gives confidence that the FR4-based PGP-TM has good pulse fidelity. As with the previous antenna designs, the waveforms resemble the first derivative of the Gaussian pulse applied to the antenna input. Thus, this result also serves as an indication of good time domain performance for the FR-4 PGP-TM. 


\subsubsection{LTCC-based Partial Ground Plane Triangular Monopole Simulation}

As with the FR-4 based PGP-TM a "staircase" approximation is used on the angled edges of the antenna and especially on the parasitic elements. Table 4.14 shows the FDTD parameters used in the simulation of the LTCC based PGP-TM. $\Delta z$ is chosen

Table 4.14: LTCC-based PGP-TM FDTD analysis parameters

\begin{tabular}{|c|c|}
\hline Increment & Value $(\mathrm{mm})$ \\
\hline$\Delta x$ & 0.450 \\
\hline$\Delta y$ & 0.500 \\
\hline$\Delta z$ & 0.396 \\
\hline
\end{tabular}

so that three Yee cells exactly match the thickness of the substrate, while $\Delta x$ and $\Delta y$ are chosen to exactly fit the width of the microstrip feed and the length of the antenna, respectively. The total mesh dimensions are $95 \times 80 \times 44$ in the $\hat{x}, \hat{y}$ and $\hat{z}$ directions respectively.

As discussed earlier, the parasitic elements on either side of the main body of the antenna have a large impact on its performance. Figure 4.36 shows the effect of parasitic element separation. The case with no parasitic elements is indicated with a dashed line. Bandwidth increases with increasing element separation, up to a point. At a distance of $1 \mathrm{~mm}$ the match is very good, while at $2.5 \mathrm{~mm}$ the match is poorer but the impedance bandwidth has increased by almost $1 \mathrm{GHz}$. A distance of $2.0 \mathrm{~mm}$ was chosen to be optimum and is indicated with a dotted line. It provides a good compromise between the depth of the $\left|S_{11}\right|$ curve and broad bandwidth. 


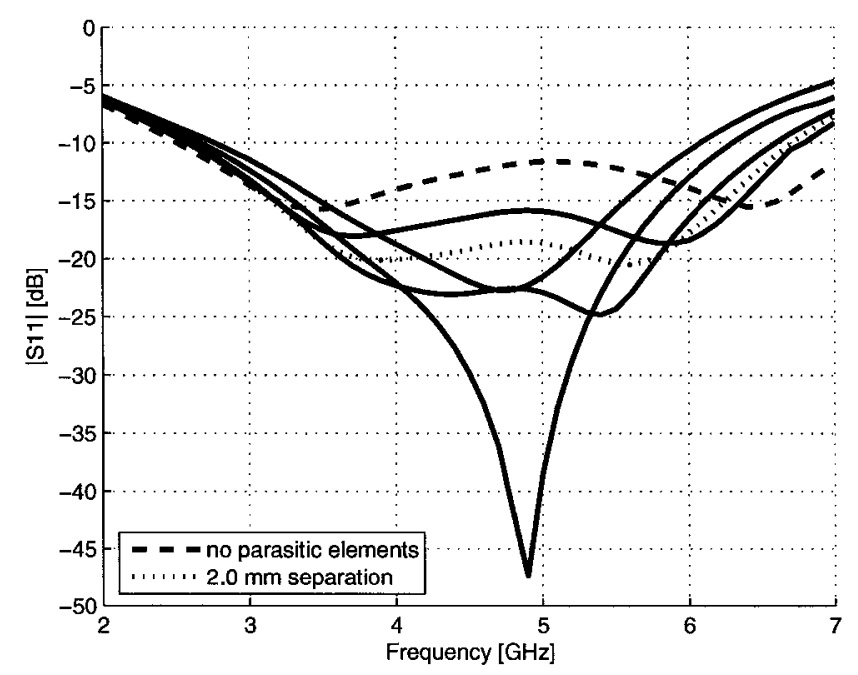

Figure 4.36: Simulated effect of parasitic element separation

Along with the response in the time domain, a comparison of the return loss generated by HFSS and FDTD for this configuration is shown in Figure 4.37. The FDTD simulation


Figure 4.37: Simulated LTCC PGP-TM return loss

results agree well with those generated by HFSS. However, the FDTD program shows a deeper minimum at $4 \mathrm{GHz}$ and a higher upper frequency limit for impedance bandwidth. 
These differences may be attributed to the decreased effectiveness of the Mur boundary condition at higher permittivities and also to the sensitivity introduced by the "staircase" approximation.

A 3-dimensional plot of the radiation pattern at $6 \mathrm{GHz}$ is shown in Figure 4.38, showing a dipole-like radiation characteristic. Moreover, the microchip circuit feature does not cause any pattern distortion. The 2-dimensional radiation characteristics at $4 \mathrm{GHz}$ and

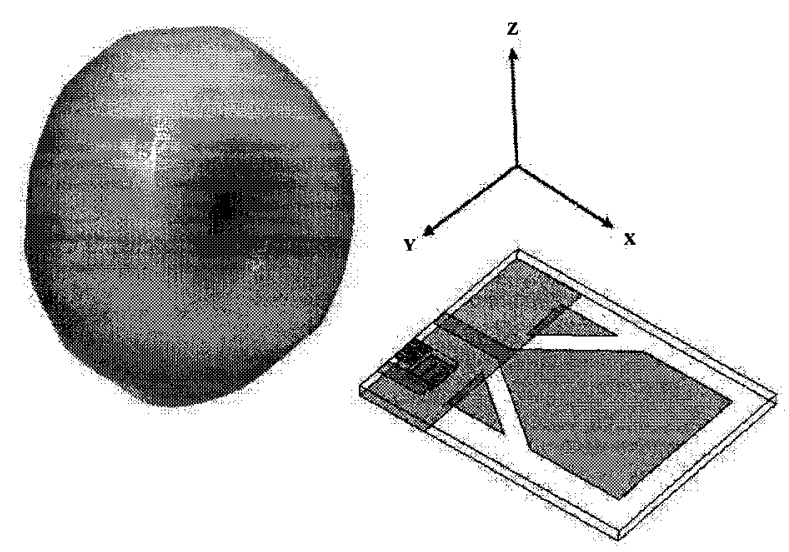

Figure 4.38: Simulated PGP-TM 3D radiation pattern at $6 \mathrm{GHz}$

$6 \mathrm{GHz}$ of the PGP-TM are displayed in Figures 4.39 to 4.42 . The substrate lies in the $\mathrm{x}-\mathrm{y}$ plane. The results show a stable omni-directional pattern with a peak gain of $2 \mathrm{~dB}$. The cross-polarization levels are shown to be below $-15 \mathrm{~dB}$ at both frequencies.

To verify the pulse fidelity of the omnidirectional PGP-TM, three different sampling locations are selected and their results are compared. The near field probes are placed to the left, middle and right of the antenna and at a height above the plane of the antenna. Sampling the co-polarized, $E_{y}$, component of the electric field at these locations results in the plot below:

The close similarity in the waveforms gives confidence that the LTCC-based PGP-TM 


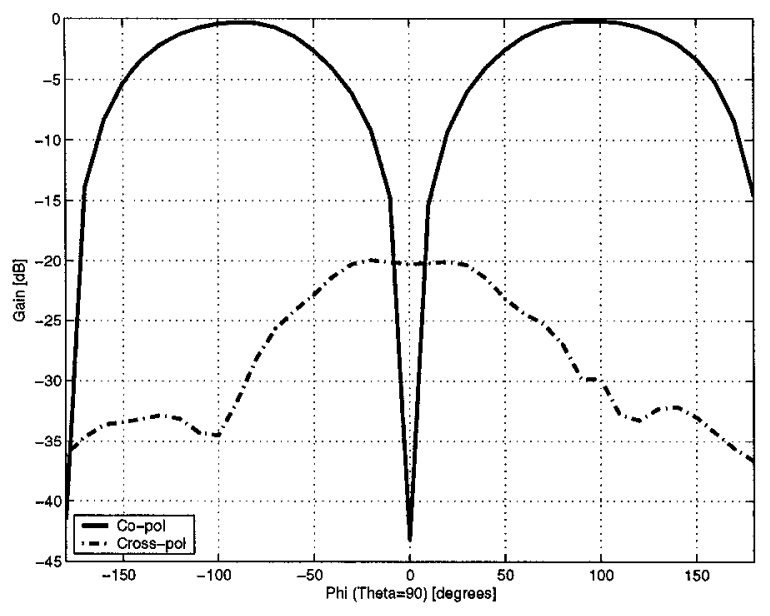

Figure 4.39: Simulated LTCC PGP-TM $4 \mathrm{GHz}$ XY-plane

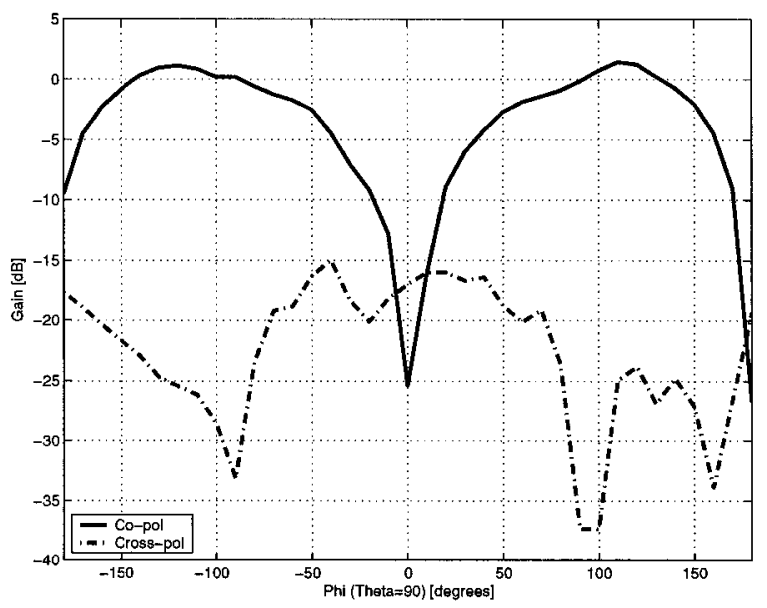

Figure 4.41: Simulated LTCC PGP-TM $6 \mathrm{GHz}$ XY-plane

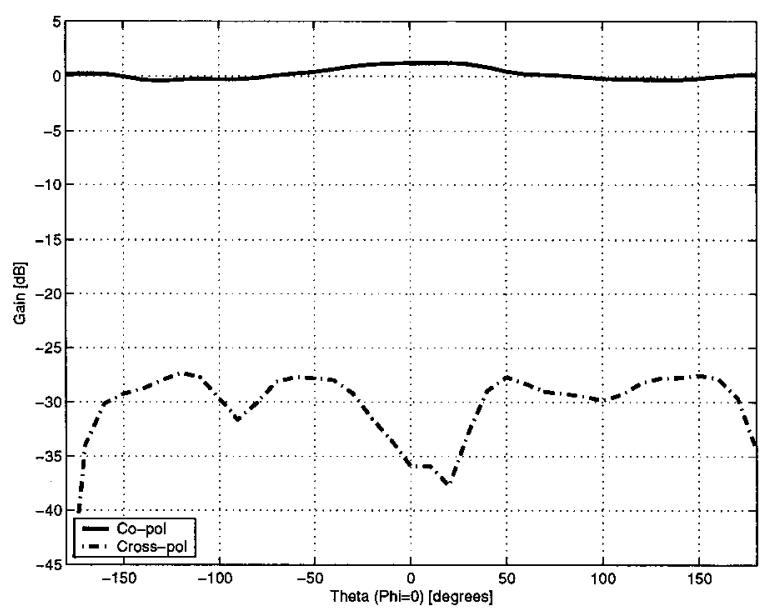

Figure 4.40: Simulated LTCC PGP-TM $4 \mathrm{GHz}$ YZ-plane

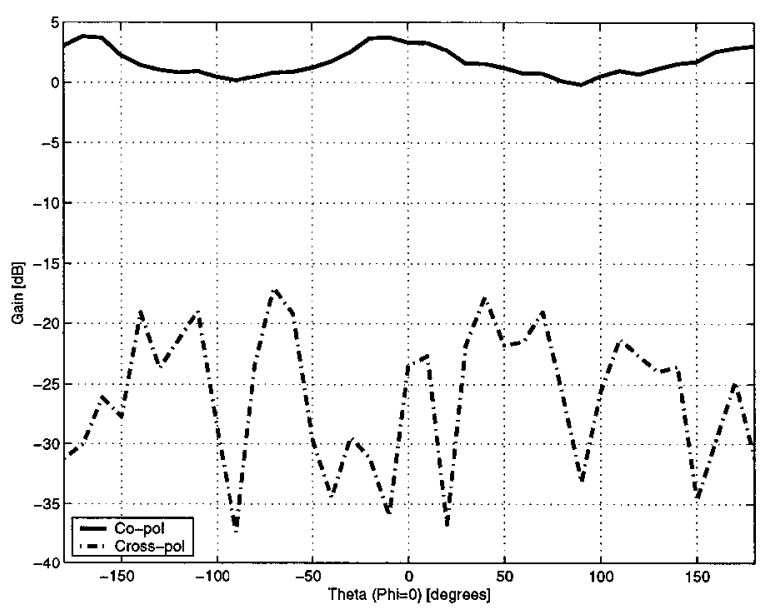

Figure 4.42: Simulated LTCC PGP-TM $6 \mathrm{GHz}$ YZ-plane 


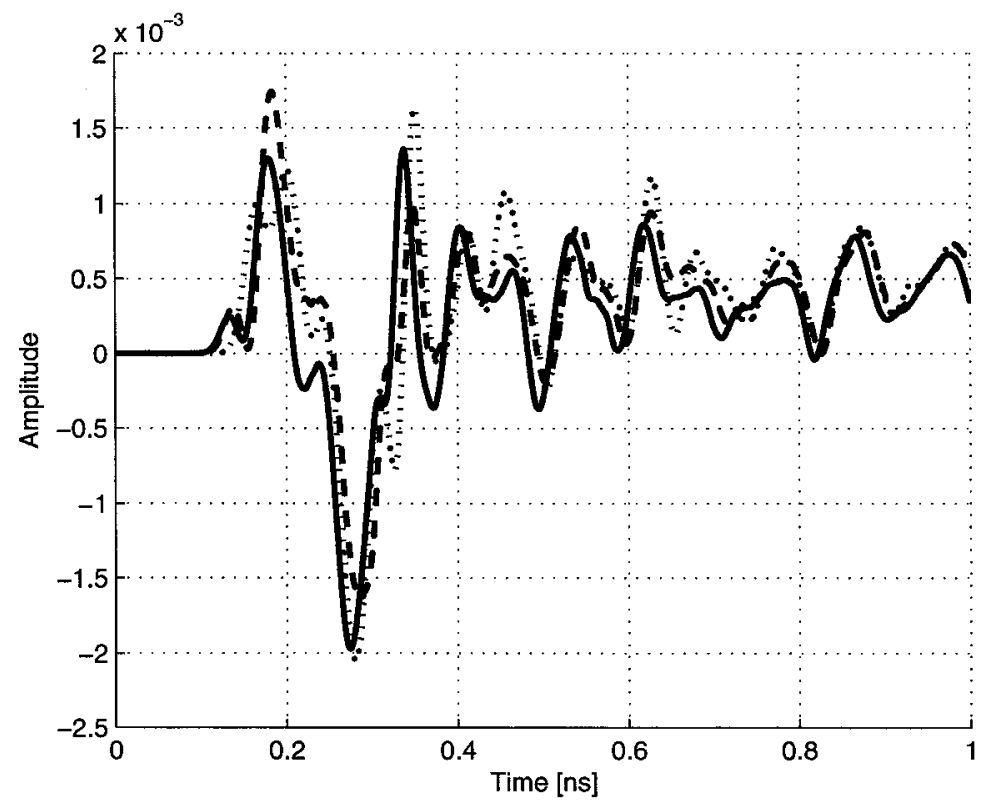

Figure 4.43: Simulated near field probe result for LTCC PGP-TM

has good pulse fidelity. As with the previous antenna designs, the waveforms resemble the first derivative of the Gaussian pulse applied to the antenna input. Thus, this result also serves as an indication of good time domain performance for the LTCC-based PGP-TM.

\subsection{Triangular Monopole Antenna with Orthogonal Ground Plane Design and Simulation}

The design of the orthogonal ground plane triangular monopole antenna (OGP-TM) served as an investigation into antennas based on very high permittivity substrates. The effects due to geometry variations and parasitic elements were also investigated. The necessity for an orthogonal ground plane and coaxial connector introduced extra complexity into the simulation and measurement stages of this design.

The OGP-TM was designed to operate in the $5 \mathrm{GHz}$ range of the radio frequency 
(RF) spectrum, making it compatible with a set of wireless local area network (WLAN) communication standards such as IEEE $802.11 \mathrm{a}(5.15 \mathrm{GHz}$ to $5.35 \mathrm{GHz})$ and HiperLAN $(5 \mathrm{GHz})$ in Europe. The complete design specifications for this antenna are provided in Table 4.15 .

Table 4.15: OGP-TM Specifications

\begin{tabular}{|c|c|}
\hline Bandwidth & $4.5 \mathrm{GHz}-5.5 \mathrm{GHz}$ \\
\hline Substrate & Experimental \\
& $\varepsilon_{r}=68$ \\
& Tan $\delta=0.002$ \\
& height $=1.0 \mathrm{~mm}$ (10 layers) \\
\hline Conductor & Silver \\
\hline
\end{tabular}

The OGP-TM consists of a main triangular metal area with two smaller adjacent parasitic elements. These elements contribute to the broadband behavior of the antenna. Three rectangular slots are made in the main triangle. The slots are designed to minimize the size of the antenna. The current on the antenna is forced to follow a longer path than without the slots, thereby making the antenna seem electrically larger. The size and position of the slots was chosen to force the current to follow close to the edge of the antenna. The same metallization is duplicated on the opposite face of the substrate. A total of thirteen vias are used to connect the two metallizations at the feed point and at the corners of the main triangle. This feature helps to make the antenna more broadband by increasing the volume of the antenna.

The antenna is mounted on a square shaped orthogonal ground plane and fed by a coaxial connector. If $d$ and $D$ represent the diameter of the inner and outer conductors, 
respectively, then the characteristic impedance of the coaxial connector is given by [45]:

$$
Z_{0}=\frac{\eta_{0}}{2.0 \pi \sqrt{\varepsilon_{r}}} \ln \left(\frac{D}{d}\right)
$$

where, $\eta_{0}=377 \Omega$ and $\varepsilon_{r}=2.1$ (teflon)

If $d$ is set to $0.375 \mathrm{~mm}$ then for a characteristic impedance of $50 \Omega D$ becomes $1.25 \mathrm{~mm}$. The center conductor of the coaxial cable is connected to the feed point of the antenna. Figure 4.44 shows the configuration of the proposed antenna with important geometrical details indicated. The perpendicular ground plane is situated in the same plane as the bottom edge of the substrate and is sized $60 \mathrm{~mm} \times 60 \mathrm{~mm}$. The sizing corresponds to $\lambda / 2$ at $5 \mathrm{GHz}$.

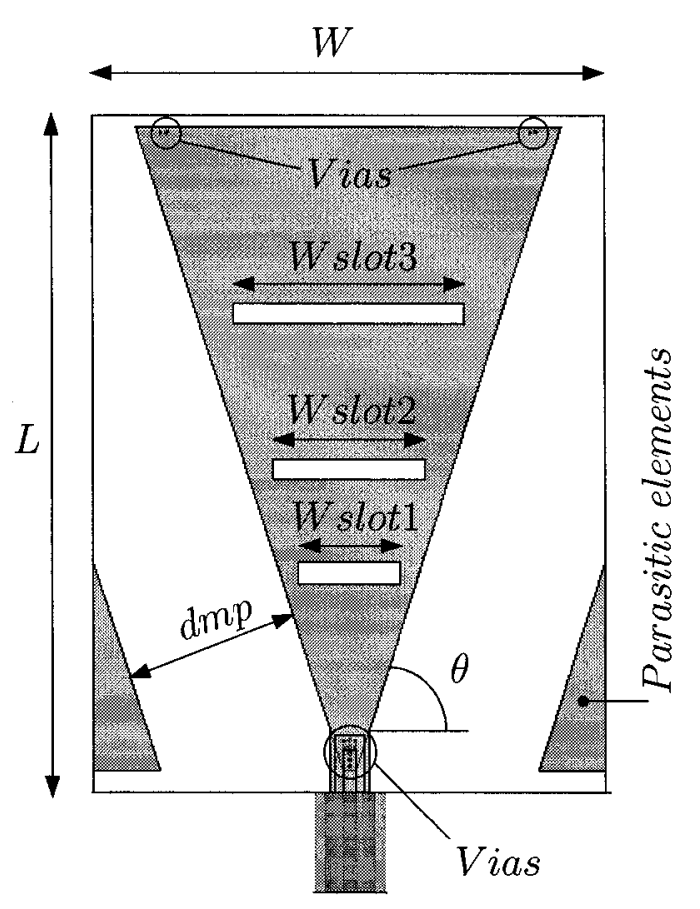

Figure 4.44: OGP-TM schematic

\begin{tabular}{|c|c|}
\hline Dimension & Value $[\mathrm{mm}]$ \\
\hline \hline $\mathrm{L}$ & 32 \\
\hline $\mathrm{W}$ & 30 \\
\hline$d_{m p}$ & 8.5 \\
\hline$w_{\text {slot } 1}$ & 5 \\
\hline$w_{\text {slot } 2}$ & 7.5 \\
\hline$w_{\text {slot } 3}$ & 11.25 \\
\hline$\theta$ & $72 \mathrm{deg}$ \\
\hline
\end{tabular}

Table 4.16: OGP-TM dimensions

The length and width of the antenna were chosen to be $32 \mathrm{~mm}$ and $30 \mathrm{~mm}$, respectively, to fit inside the area allocated on the LTCC substrate. The flare angle and parasitic 
element separation were optimized to give the broadest impedance bandwidth and are $72 \mathrm{deg}$ and $8.5 \mathrm{~mm}$, respectively. A section of the coaxial connector was modeled to make the simulation as realistic as possible. This antenna was not modeled in the FDTD based electromagnetic simulator because of the extra complexity associated with the coaxial connector.

\subsubsection{Orthogonal Ground Plane Triangular Monopole Simula- tion}

The purpose of this antenna is to investigate the effect that extremely high permittivities have on antenna performance. HFSS was used to simulate the antenna along with a portion of the coaxial connector. A plot of the return loss is shown in Figure 4.45. The

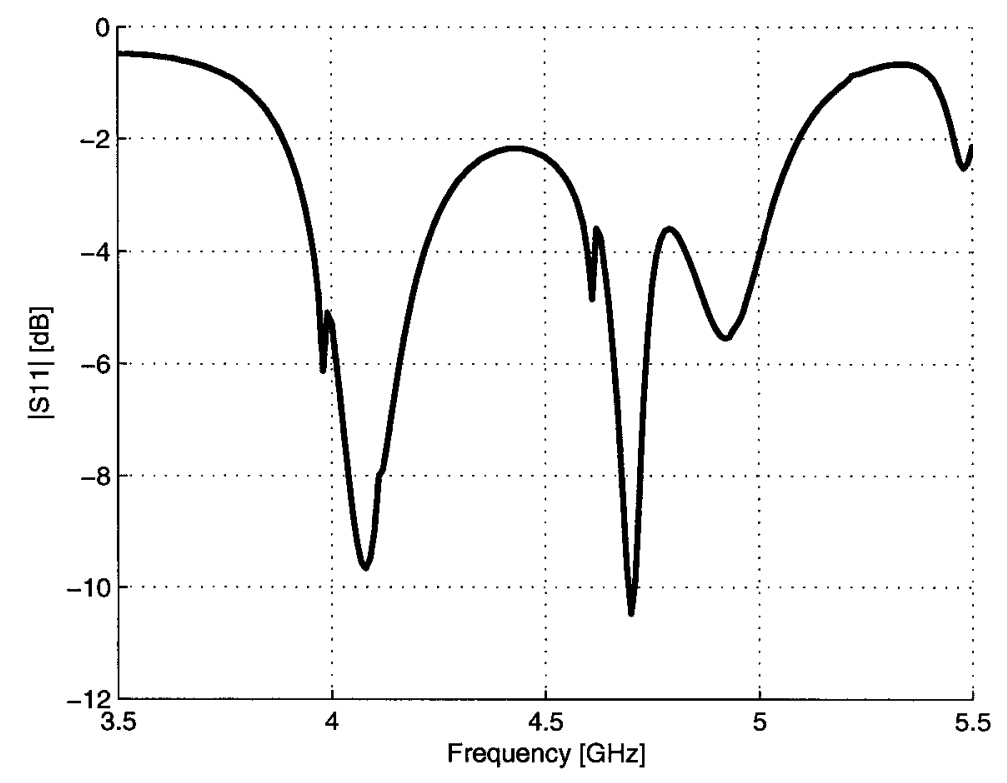

Figure 4.45: OGP-TM Return Loss

plot indicates two regions of resonance at $4.08 \mathrm{GHz}$ and $4.7 \mathrm{GHz}$.

For the radiation pattern simulations, the antenna's ground plane was placed in the 
xy-plane, making the axis of the antenna oriented along the z-axis. Two principal plane cuts were generated at $4.7 \mathrm{GHz}$ and are shown in Figures 4.46 and 4.47 . The results show an omni-directional radiation pattern in the xy-plane plane with a peak gain of $2 \mathrm{~dB}$. Cross-polarization levels are $10 \mathrm{~dB}$ below the peak co-polarization levels.

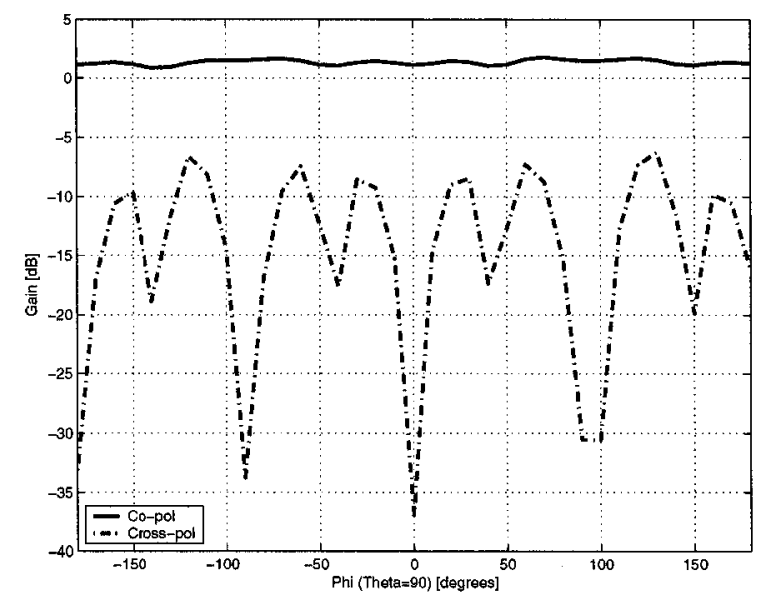

Figure 4.46: OGP-TM 4.7 GHz XY-plane

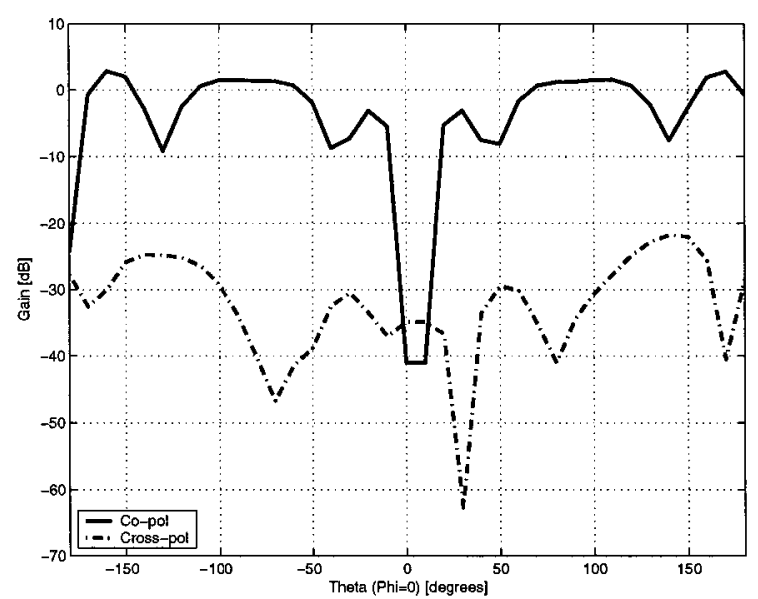

Figure 4.47: OGP-TM 4.7 GHz XZ-plane

\subsection{Planar Circuit Integration}

The LTCC versions of the AVA and PGP-TM antennas were designed with RF circuit integration in mind. To this end, a circuit feature was added to the designs that could accommodate a microchip die. Figures 4.48 and 4.49 show the LTCC antennas with the circuit feature added. Importantly, the antennas can operate with or without a functional microchip die. Figures 4.50 and 4.51 show the circuit in detail.

The circuit feature is placed $3.25 \mathrm{~mm}$ away from the adjacent microstrip feed line of the antenna to minimize interference. The circuit feature consists of a cavity with a grounded floor. The cavity has a footprint of $3 \mathrm{~mm} \times 4 \mathrm{~mm}$ to ensure that even large microchip dies can be used. Metal traces were drawn along one edge of the cavity. In this way, a 


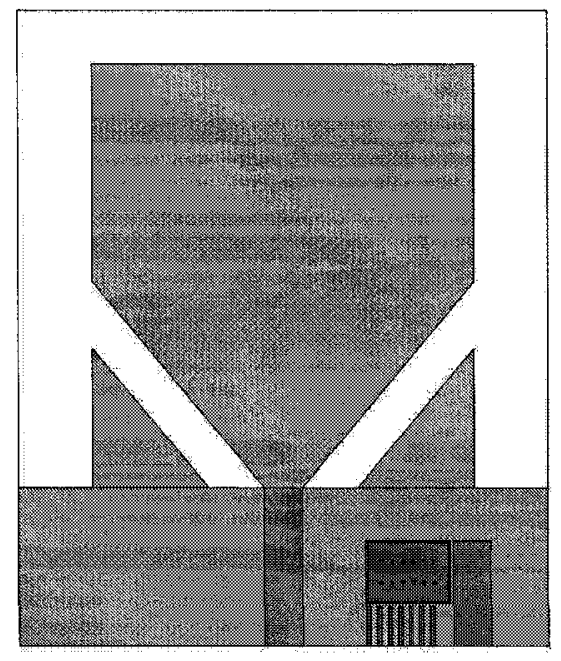

Figure 4.48: PGP-TM with module

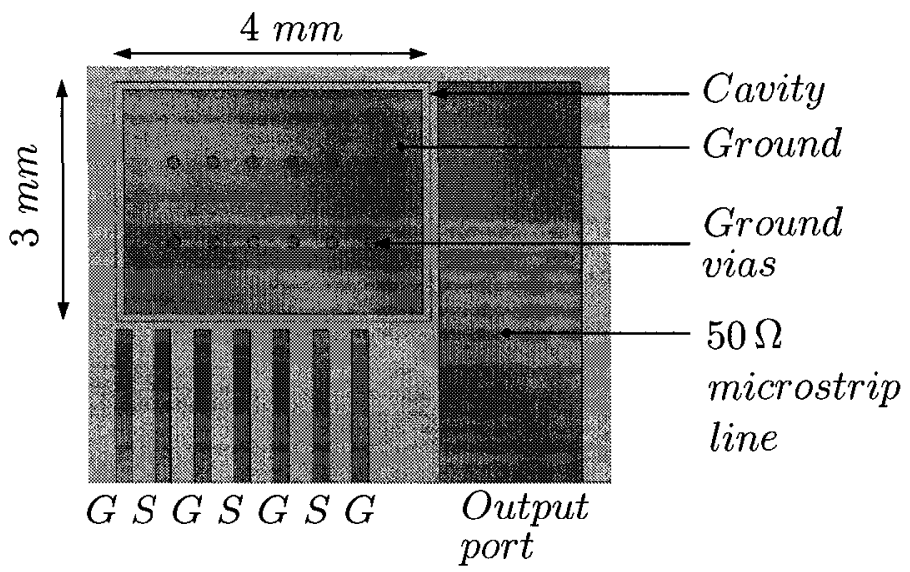

Figure 4.50: Circuit module (top)

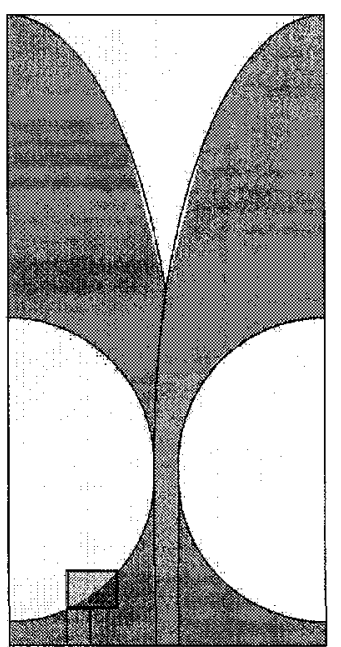

Figure 4.49: AVA with module

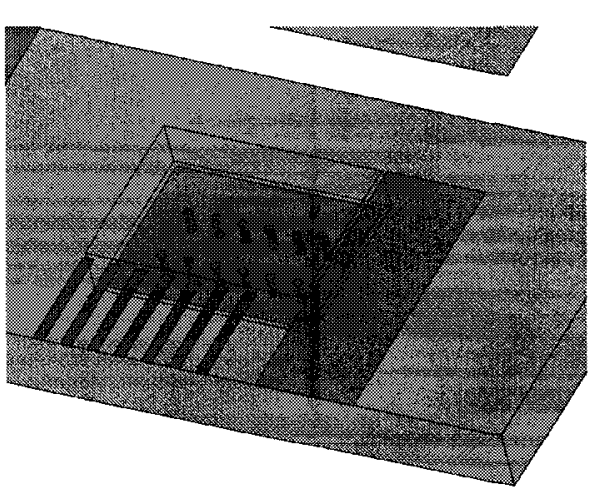

Figure 4.51: Circuit module (angled) 
daughter board could be easily attached to the LTCC substrate to feed ground and power to the microchip. A second $50 \Omega$ microstrip line was added to serve as an alternate output port. The microchip itself would be placed inside the cavity and bonded to the ground plane with conductive epoxy. Minimum length bond wires would be used to connect the microchip to the output of the antenna.

These designs show that LTCC technology can be used to combine an antenna and RF electronics to form a compact and low cost UWB transceiver.

\subsection{Simulation Summary}

Six different antennas were designed and simulated, including a B-AVA, OGP-TM, FR-4 and LTCC versions of the AVA and PGP-TM. The agreement between the HFSS and FDTD $\left|S_{11}\right|$ results is good. The radiation patterns generated by HFSS are what was expected of the antenna designs. Furthermore, the circuit feature for a UWB transceiver chip integrated with the LTCC antennas does not seem to degrade performance.

Novel parasitic elements were introduced and their effect on the performance of the PGP-TM was investigated. The results revealed that these elements reduce the input impedance variation with frequency of the antenna and thus can be used as an important design parameter.

Near field probe simulations were performed to obtain an understanding of the time domain radiated electric fields for each antenna. As expected, all the results indicated a waveform that resembled the first derivative of the applied Gaussian pulse. To verify the pulse fidelity of the omni-directional PGP-TM antennas, three different sampling 
locations around the antennas were selected and their results compared. Sampling the co-polarized components of the electric fields at those locations resulted in only small variations, indicating good pulse fidelity. 


\section{Chapter 5}

\section{UWB Antenna Measurements and Comparisons}

This chapter provides an extensive amount of measurements for the UWB antennas presented in Chapter 4. Time and frequency domain measurements are conducted to verify the simulated results and determine the tradeoffs between these designs. The simulated and measured results are compared and their degree of agreement is discussed.

\subsection{Measurement Setup}

Anechoic chamber measurements are made by placing the antenna under test at the near end of the chamber, while placing a standard gain horn antenna at the far end (see Figure 5.1). The equipment used included an Agilent $8720 \mathrm{ES}$ vector network analyzer and a computer workstation running FR959 far-field measurement software. Two principle plane measurements, azimuth and elevation, are taken at 4, 4.7,6 and $8 \mathrm{GHz}$. Based on spherical coordinates, an azimuthal plane measurement constitutes rotation in the xyplane, which is the plane of the antenna. An elevation plane measurement is performed in the orthogonal xz-plane. Both co-polarized and cross-polarized components are to be 


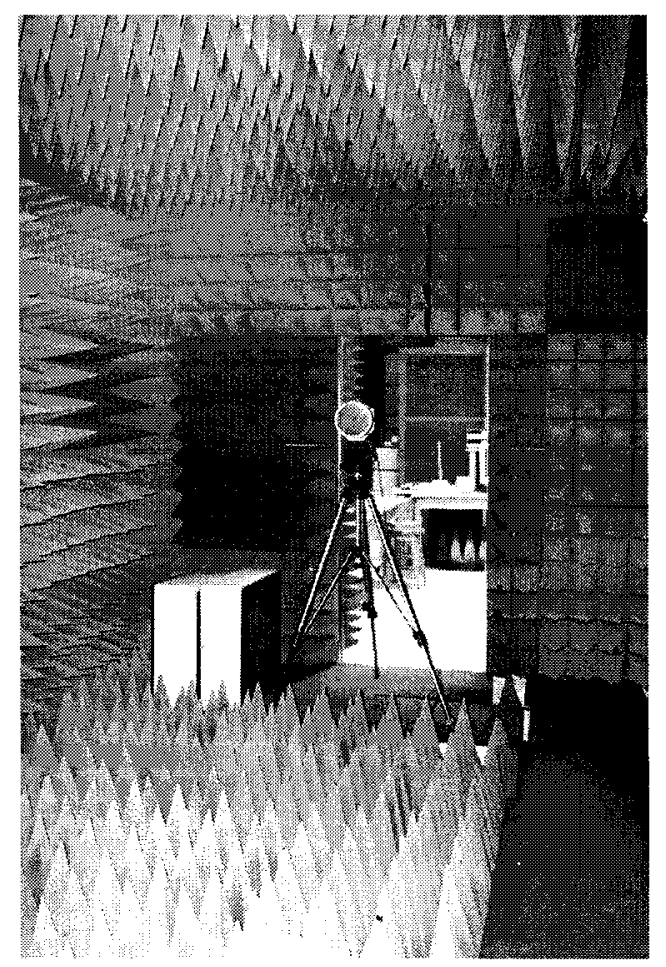

Figure 5.1: Anechoic chamber

obtained. The antennas measured are listed below:

- Orthogonal ground plane triangular monopole

- FR4-based antipodal Vivaldi

- FR4-based partial ground plane triangular monopole

- $\quad$ LTCC-based antipodal Vivaldi

- LTCC-based partial ground plane triangular monopole

In addition to radiation patterns, scattering parameter measurements are to be performed within a Faraday cage to minimize environmental interference. First the $\left|S_{11}\right|$ of each antenna is obtained to verify broadband impedance matching. Then the $S_{21}$ of a two antenna system (using identical antennas under test) is measured to assess linear phase and flat amplitude response. Measurements are performed at $20 \mathrm{~cm}$ and $75 \mathrm{~cm}$ to confirm 
the results. An ideal base-line for the $S_{21}$ measurements is created by directly connecting two ports of the network analyzer.

Time domain measurements are performed at CRC using a burst CW UWB pulse generator, Agilent $86100 \mathrm{~A}$ wide-bandwidth oscilloscope and a computer workstation. The receive and transmit antennas are mounted on plastic columns and placed $50 \mathrm{~cm}$ apart. The transmit and receive antennas are connected with high quality cables to the signal generator and oscilloscope, respectively. To create a near-ideal benchmark case, a cable is used to directly connect the pulse generator to the oscilloscope. A burst CW UWB pulse centered at $6 \mathrm{GHz}$ and having a bandwidth of $2 \mathrm{GHz}$ is employed [55]. The spectral content (normalized to peak power) of the burst CW pulse satisfies the UWB spectrum mask as defined by the FCC. The pulse and its corresponding spectral content are shown in Figure 5.2. To achieve a meaningful comparison between the different pulse responses,
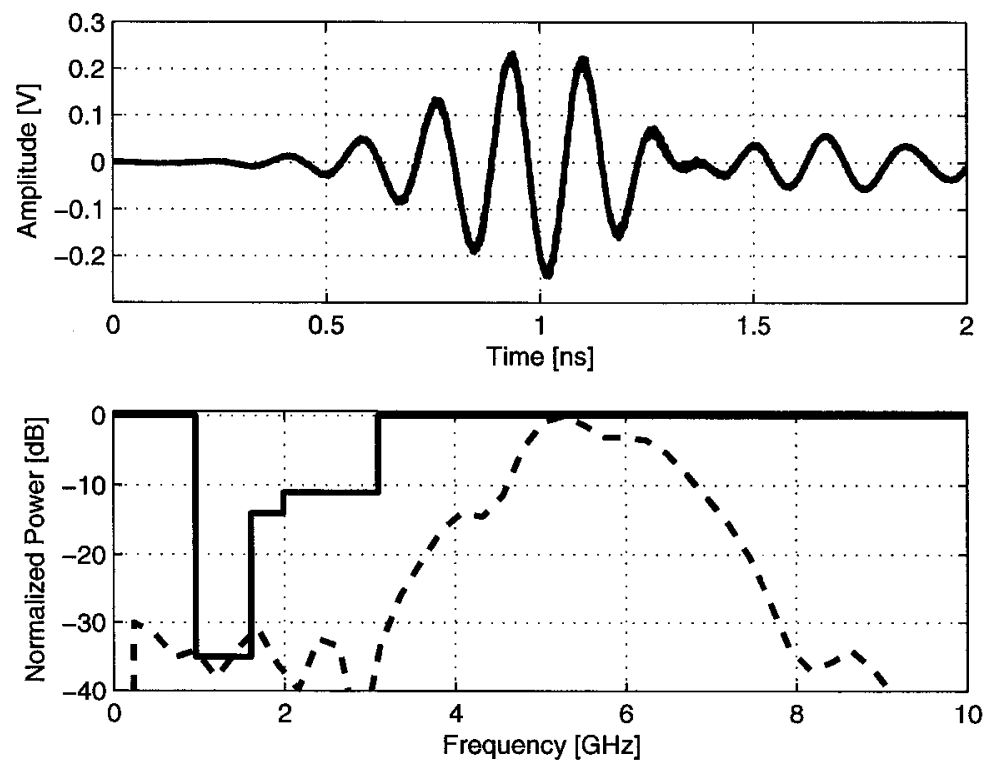

Figure 5.2: Measured time domain pulse and spectral content 
the pulses obtained from the antenna systems are scaled to match the peak amplitude of $230 \mathrm{mV}$ of the benchmark case. In this way, the differences in the relative pulse shapes can be easily seen.

\section{2 $5 \mathrm{GHz}$ Series Patch Array}

Before characterizing the UWB antennas of this thesis, it is useful to examine the response in the time domain of a narrow band antenna, as was done in Chapter 3 . To this end, a $5 \mathrm{GHz}$ patch antenna is measured (with a receive antenna known to exhibit good broadband performance). Figure 5.3 plots the input return loss, confirming the very narrowband performance of this antenna at $5 \mathrm{GHz}$. The response in the time domain is



Figure 5.3: Measured $\left|S_{11}\right|$ for narrowband patch antenna

shown in Figure 5.4 along with the applied pulse. As expected the response is very poor, being almost sinusoidal in nature and, although not shown, late-time ringing is significant 
up to $5 \mathrm{~ns}$. Moreover, the received pulse is severely attenuated with a peak amplitude of only $1.59 \mathrm{mV}$, much smaller than the other cases to be shown later. This result shows

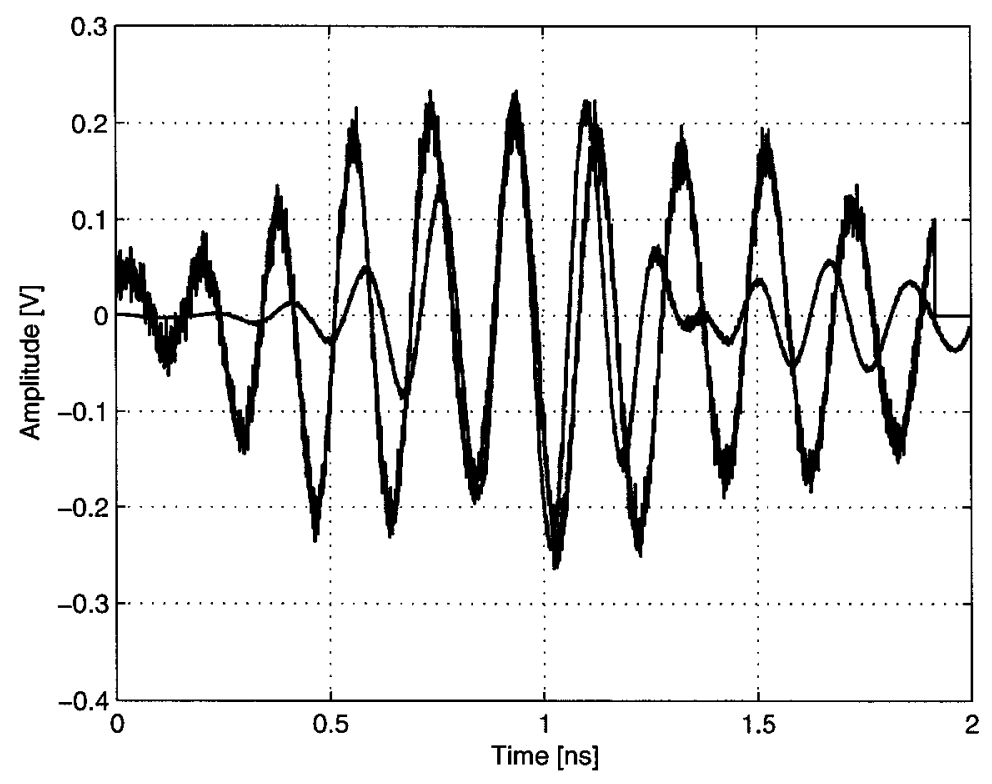

Figure 5.4: Measured response in the time domain for the narrowband patch antenna

that a narrowband antenna is completely unsuitable for a UWB antenna application.

\subsection{Orthogonal Ground Plane Triangular Monopole}

A photograph of the OGP-TM mounted on a partial ground plane, with dimensions of $6 \mathrm{~cm} \times 6 \mathrm{~cm}$, is shown in Figure 5.5. The total volume occupied by the antenna now becomes $6 \mathrm{~cm} \times 6 \mathrm{~cm} \times 32 \mathrm{~cm}$ or $1152 \mathrm{~cm}^{3}$

First, the return loss and radiation patterns are obtained in the frequency domain. Figure 5.6 shows the simulated and measured return loss results. The measurements indicate an impedance bandwidth from $4.5 \mathrm{GHz}$ to $5.64 \mathrm{GHz}$ with a peak $\left|S_{11}\right|$ of $-8.0 \mathrm{~dB}$ at $5.14 \mathrm{GHz}$. However, there is a noticeable discrepancy with the simulated values that 


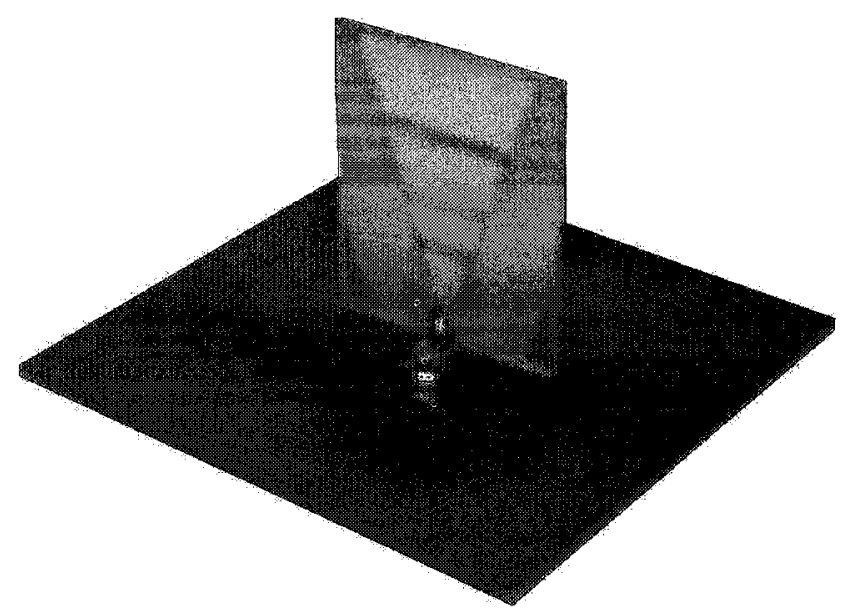

Figure 5.5: LTCC OGP-TM

is most likely due to improper modeling of the SMA connection at the feed point of the antenna. Reflections at this interface can easily reduce the accuracy of the simulated results. Nonetheless, a resonance appears at $4.7 \mathrm{GHz}$ in both the measured and simulated data. These results show that, although not as broadband as the AVA or PGP-TM, the OGP-TM still covers an extensive bandwidth as required for several WLAN standards.

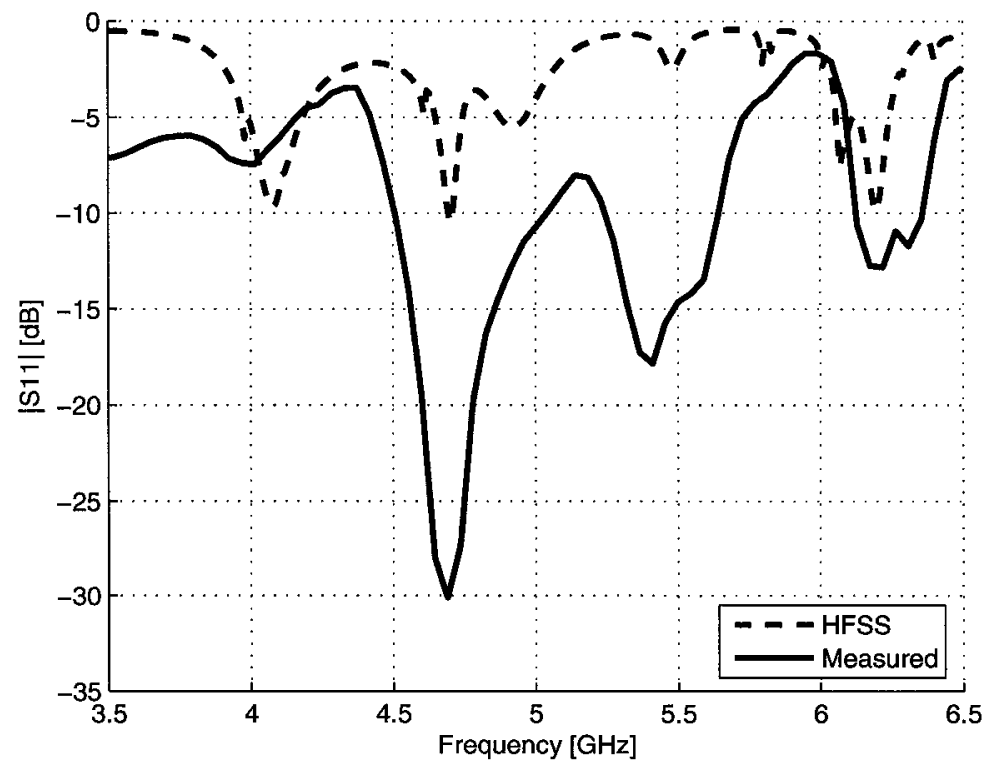

Figure 5.6: OGP-TM $\left|S_{11}\right|$ measurement 
The radiation pattern results for the two principal pattern cuts at $4.7 \mathrm{GHz}$ are shown in Figures 5.7 and 5.8. The simulated and measured results are in good agreement with two notable exceptions. The measured azimuthal co-polarized pattern shows a larger gain variation than the simulated pattern. This can be attributed to effects caused by the SMA connector or antenna mount that were not properly modeled in the simulator. The other discrepancy is the higher than expected cross-polarization levels in the elevation plane. This discrepancy is also present in the other antenna results and may be attributed to a misalignment of the transmit antenna during measurement.

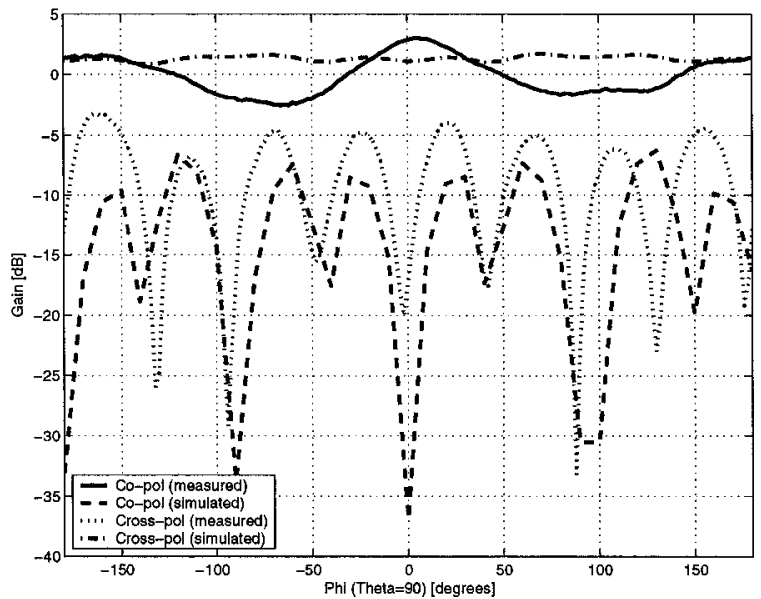

Figure 5.7: Measured azimuthal patterns for OGP-TM at $4.7 \mathrm{GHz}$

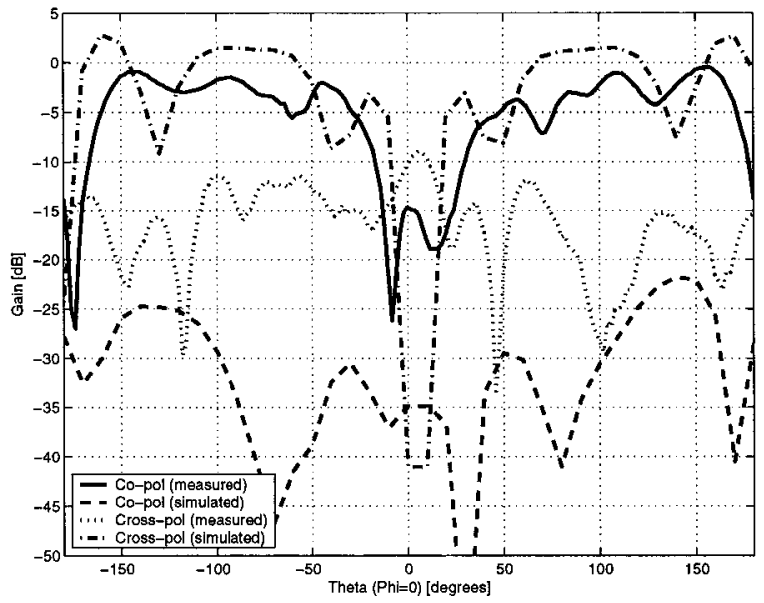

Figure 5.8: Measured elevation patterns for OGP-TM at $4.7 \mathrm{GHz}$

The response in the time domain of the OGP-TM is somewhat improved from the result obtained by the patch antenna and is shown in Figure 5.9. However, there are still significant differences in the relative amplitudes of the various peaks. Additionally, late time ringing is still present with this antenna. 


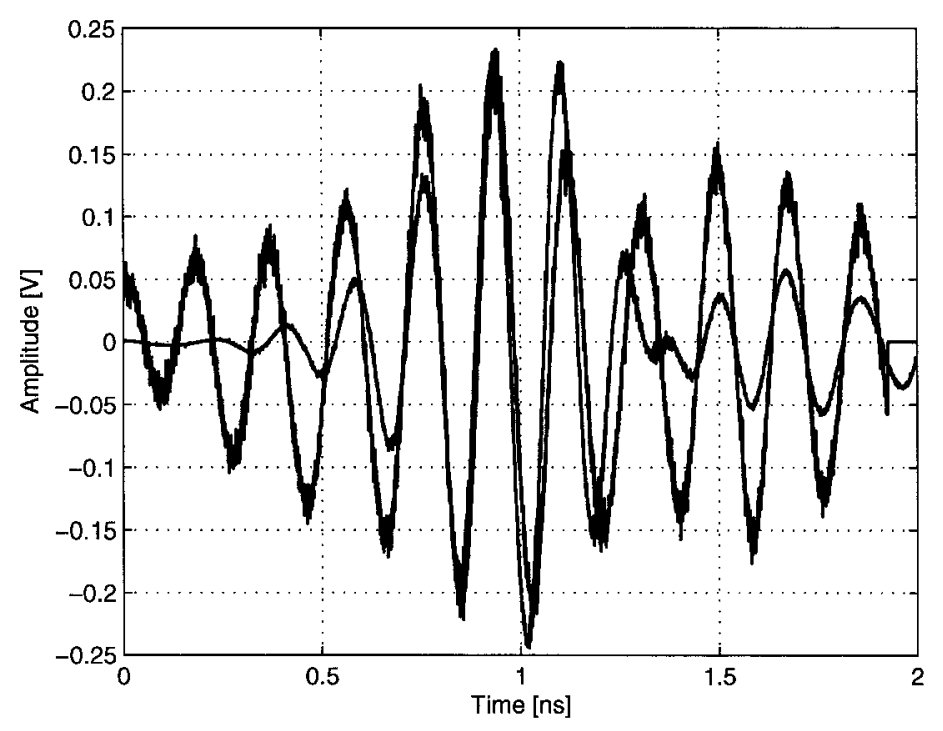

Figure 5.9: Measured OGP-TM time domain response

\subsection{FR4-based Antipodal Vivaldi}

A photograph of the FR4-based antipodal Vivaldi, including the gold SMA connector, is shown in Figure 5.10. The measured and simulated return loss results of Figure 5.11 show

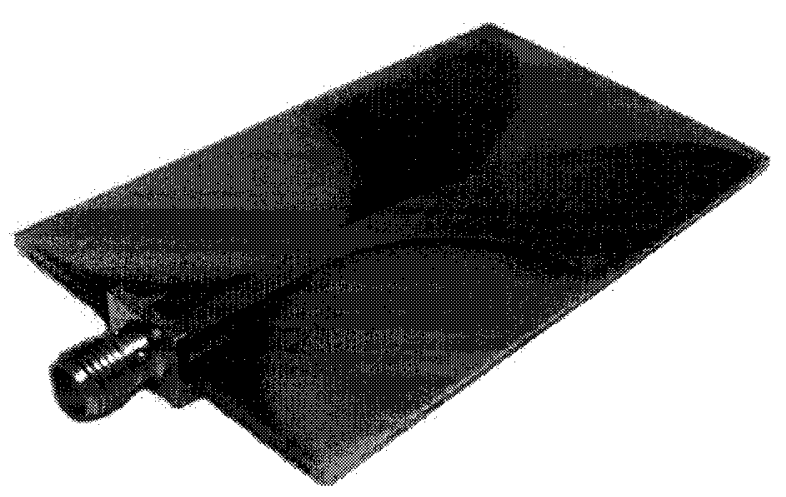

Figure 5.10: FR-4 based AVA

good agreement and testify to the accuracy of the simulated results. The measurements show an impedance bandwidth of $6 \mathrm{GHz}$ extending from $4 \mathrm{GHz}$ to $10 \mathrm{GHz}$.

The $S_{21}$ measurements were obtained from a two antenna system consisting of identical 


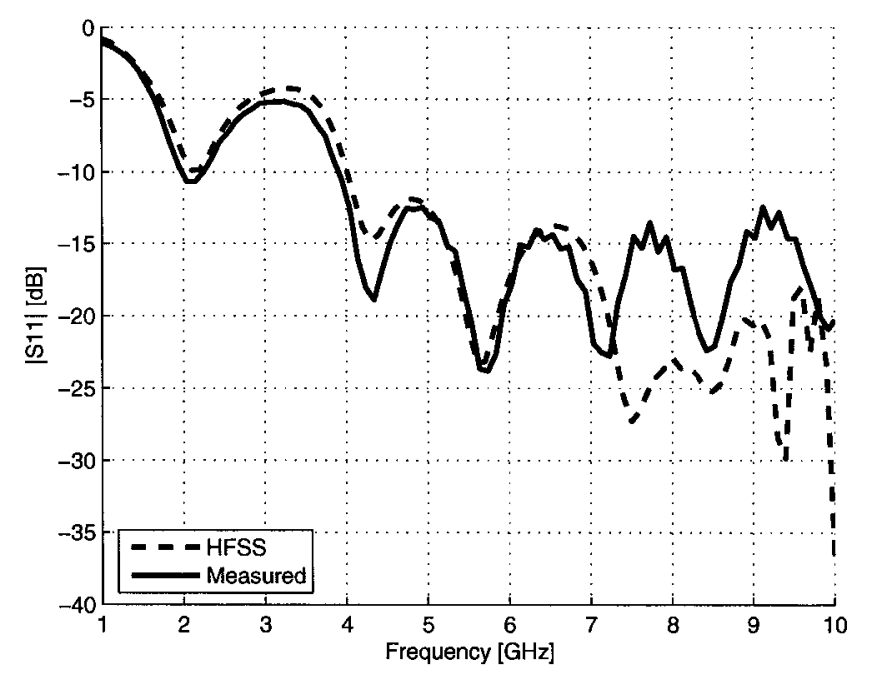

Figure 5.11: Measured $\left|S_{11}\right|$ for FR-4 based AVA

transmit and receive AVAs. A line of best fit, with equation $y=0.59 x-32$, is used to obtain the best prediction for the trend with frequency of $\left|S_{21}\right|$ from $1 \mathrm{GHz}$ to $10 \mathrm{GHz}$. Figure 5.12 shows that by using the line of best fit, $\left|S_{21}\right|$ stays within a variation of $3.2 \mathrm{~dB}$ in the antenna's operating bandwidth of $4 \mathrm{GHz}$ to $10 \mathrm{GHz}$. Over a decade $\left|S_{21}\right|$ increases
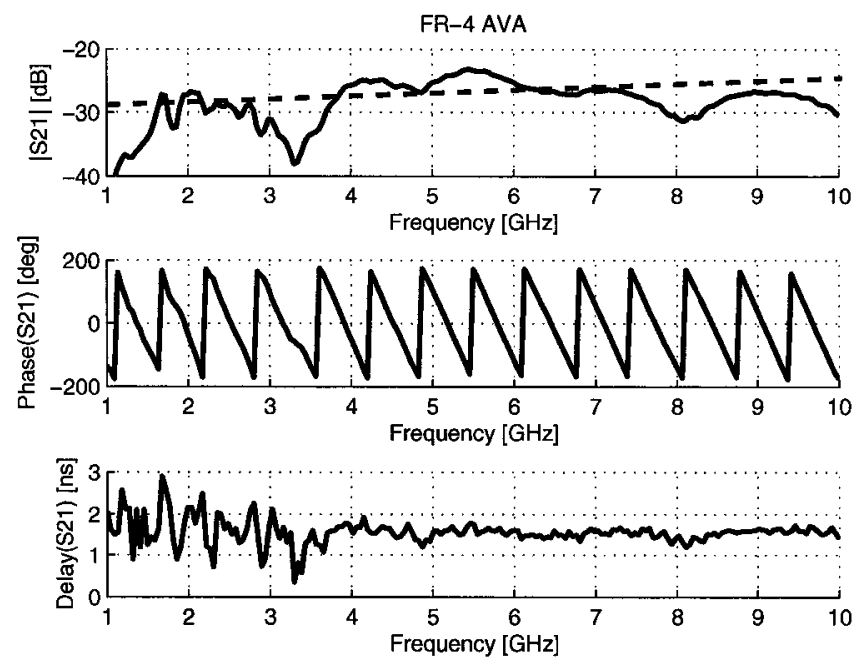

Figure 5.12: Measured $S_{21}$ of FR-4 based AVA 
as $6 \mathrm{~dB}$, however based upon the discussion in section 2.6 .2 the expected result is $20 \mathrm{~dB}$. A likely reason for this discrepancy is the fact that the substrate becomes very lossy beyond $6 \mathrm{GHz}$ and limits the gain increase. The phase of $S_{21}$ is linear in the operating bandwidth and leads to a group delay that has a maximum variation of only $728 \mathrm{ps}$.

Radiation pattern cuts at $4 \mathrm{GHz}$ and $8 \mathrm{GHz}$ in the two principle planes are shown in Figures 5.13 to 5.16 .

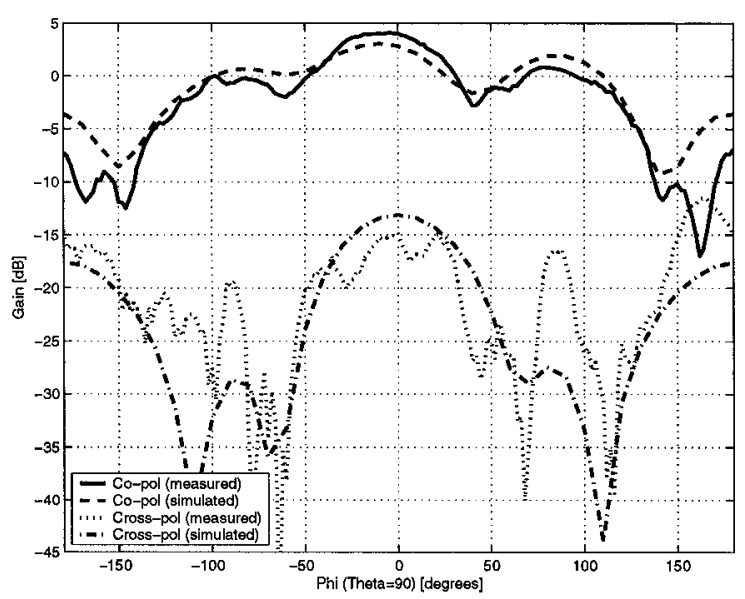

Figure 5.13: Measured azimuthal patterns for FR-4 AVA at $4 \mathrm{GHz}$

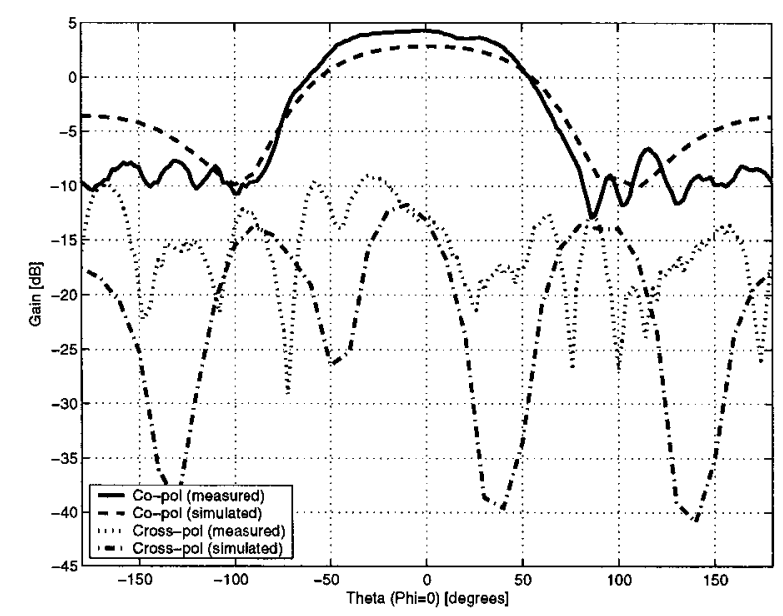

Figure 5.14: Measured elevation patterns for FR-4 AVA at $4 \mathrm{GHz}$

The agreement at $4 \mathrm{GHz}$ between the simulated and measured results is very good. The measurements indicate a boresight gain of $4.32 \mathrm{~dB}$ at $4 \mathrm{GHz}$ and $3 \mathrm{~dB}$ at $8 \mathrm{GHz}$ along with cross-polarized levels below $-10 \mathrm{~dB}$ at both frequencies. However, at $8 \mathrm{GHz}$ the agreement is notably poorer with regards to the cross-polarized levels and gain. The pattern becomes distorted and the cross-polarized levels are almost matching the co-polarized levels. These effects can be caused by the FR-4 substrate, which may indicate that the cut-off frequency has been reached. A measurement summary of the radiation pattern results is shown in Table 5.1. 




Figure 5.15: Measured azimuthal patterns for FR-4 AVA at $8 \mathrm{GHz}$

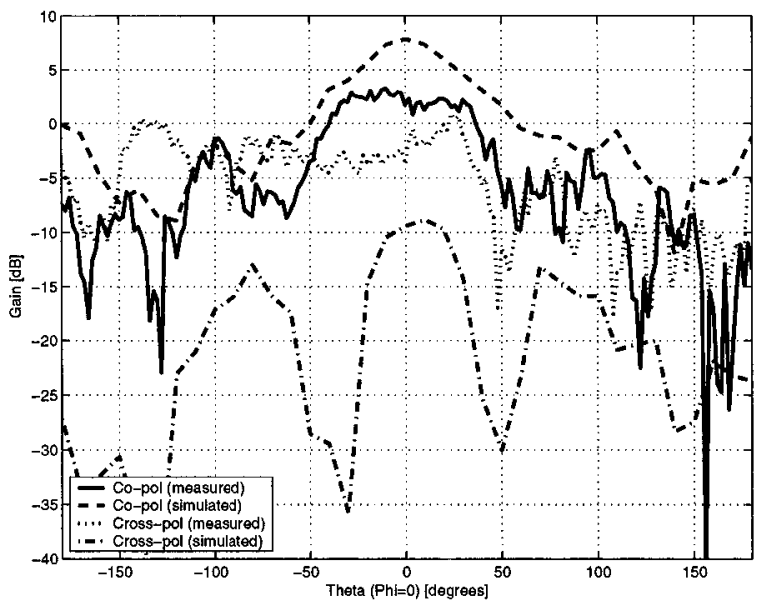

Figure 5.16: Measured elevation patterns for FR-4 AVA at $8 \mathrm{GHz}$

Table 5.1: Radiation pattern results summary for FR-4 based AVA

\begin{tabular}{|c|c|c|c|c|}
\hline & \multicolumn{2}{|c|}{$4 \mathrm{GHz}$} & \multicolumn{2}{c|}{$8 \mathrm{GHz}$} \\
\cline { 2 - 5 } & Simulated & Measured & Simulated & Measured \\
\hline HPBW (deg) & 125 & 112 & 65 & 75 \\
Gain (dB) & 3.09 & 4.32 & 7.85 & 3 \\
FBR (dB) & 6.69 & 13.93 & 7.97 & 5.77 \\
Cross-pol (dB) & -15 & -17 & -17 & -5 \\
\hline
\end{tabular}

The minimal variation in the group delay helps this antenna produce a pulse shape that is very well maintained with little dispersion or distortion visible in the received pulse. The peak amplitude of the received pulse is $7.1 \mathrm{mV}$. While these results show that the AVA has a large impedance bandwidth, good gain, linear phase and good time domain performance, the large size and lossy substrate make this antenna less attractive as a candidate for a UWB antenna application. 


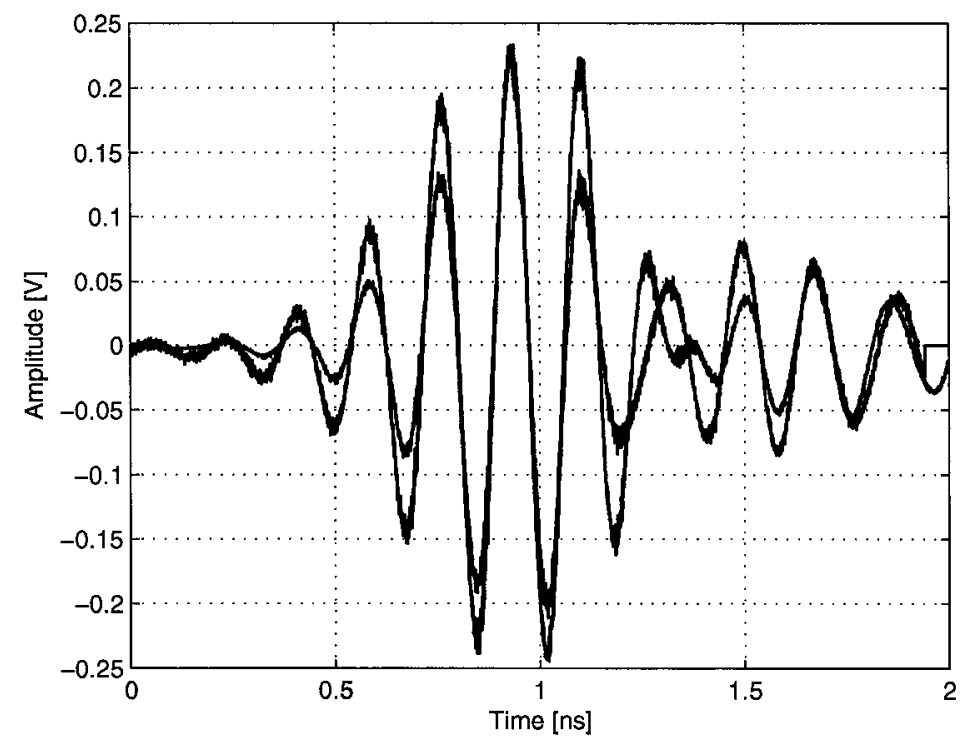

Figure 5.17: Measured FR-4 based AVA time domain response

\subsection{FR-4 based Triangular Monopole with Partial Ground Plane}

A photograph of the FR4-based PGP-TM, including gold SMA connector, is shown in

Figure 5.18. The measured and simulated return loss is shown in Figure 5.19. The

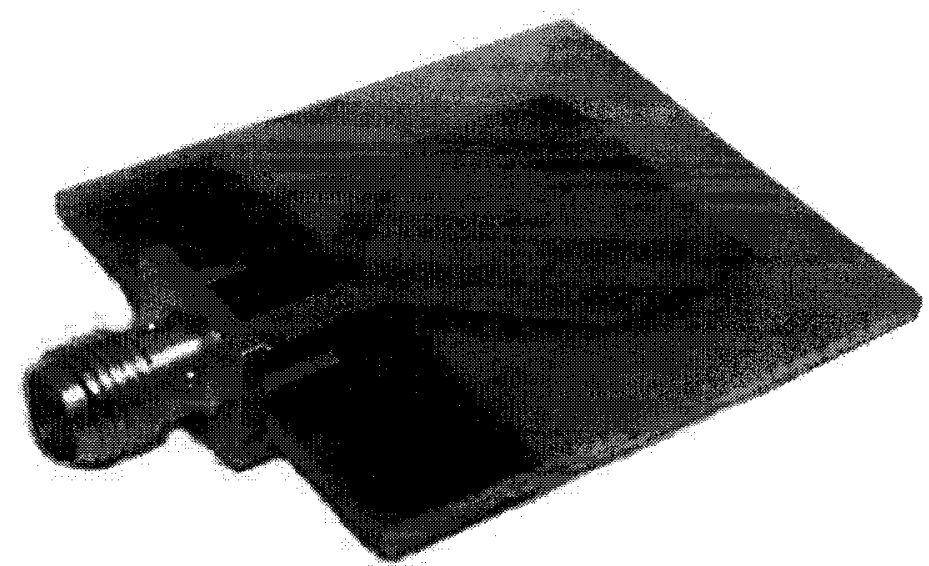

Figure 5.18: FR-4 based PGP-TM

measured result shows two regions where the $\left|S_{11}\right|$ curve is below $-10 \mathrm{~dB}$, from $3.47 \mathrm{GHz}$ 
to $6.82 \mathrm{GHz}$ and from $8.45 \mathrm{GHz}$ to $10 \mathrm{GHz}$. This gives a total bandwidth of $4.9 \mathrm{GHz}$. The agreement between HFSS and the measured results is very good. However, HFSS underestimates the lower limit of the impedance bandwidth by about $500 \mathrm{MHz}$ and does not predict the second resonance beyond $8 \mathrm{GHz}$. This discrepancy can be attributed to effects caused by the SMA connector, which was not modeled, the solder connection and extra substrate losses.

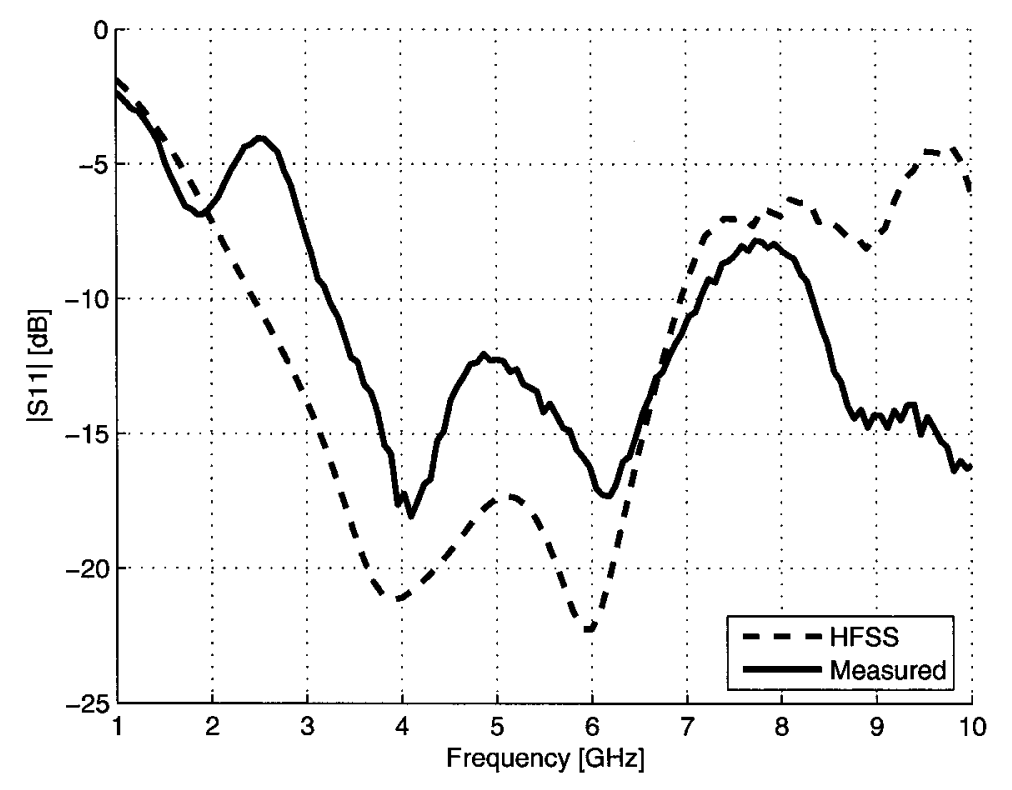

Figure 5.19: Measured $\left|S_{11}\right|$ for FR-4 based PGP-TM

Figure 5.20 shows the measured $S_{21}$ results for a two antenna system of PGP-TMs. Lines of best fit are included to show the best prediction for the relationship of $\left|S_{21}\right|$ with frequency and the variation with frequency of the group delay. According to the line of best fit, whose equation is $y=1.8 x-30,\left|S_{21}\right|$ stays within a variation of $15 \mathrm{~dB}$ in the antenna's operating band and shows a decreasing trend with frequency of $18 \mathrm{~dB}$ per decade. This measurement is in very good agreement with the expected result discussed in 
section 2.6.2. The phase of $S_{21}$ is linear beyond $4.5 \mathrm{GHz}$. Even with some initial instability before $4.5 \mathrm{GHz}$, the group delay stays within a variation of 2 ns in the operating band of the antenna when a $7^{\text {th }}$ degree polynomial is fitted to the data.

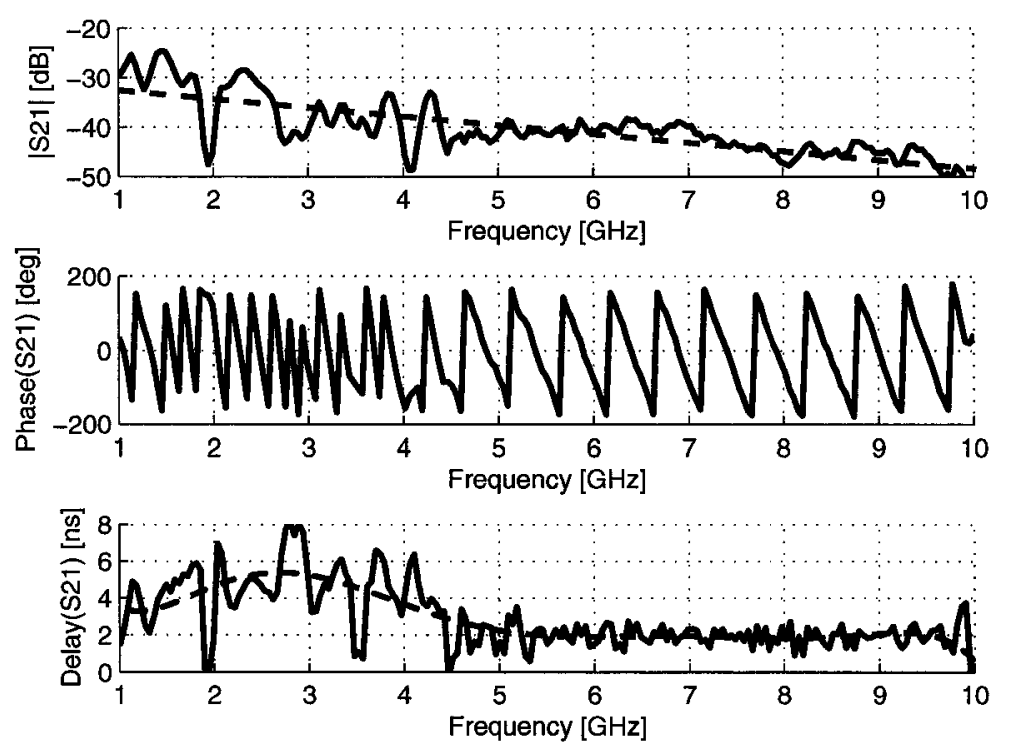

Figure 5.20: Measured $S_{21}$ of FR-4 based PGP-TM

Radiation pattern cuts at $4 \mathrm{GHz}$ and $6 \mathrm{GHz}$ in the two principal planes are shown in Figures 5.21 to 5.24. They indicate monopole-like patterns with omni-directional radiation in elevation, a gain of approximately $0 \mathrm{~dB}$ and cross-polarized levels below $-5 \mathrm{~dB}$ at $4 \mathrm{GHz}$ and below $-10 \mathrm{~dB}$ at $6 \mathrm{GHz}$. The similarities in the radiation patterns at both frequencies indicate good pattern stability across the entire impedance bandwidth. The simulated antenna gain is slightly overestimated due to extra dielectric losses and mismatch at the SMA connector.

As shown in Figure 5.25, the response in the time domain of this antenna shows very little distortion between the received pulse and that of the ideal case. The peak 




Figure 5.21: Measured azimuthal patterns for FR-4 PGP-TM at $4 \mathrm{GHz}$

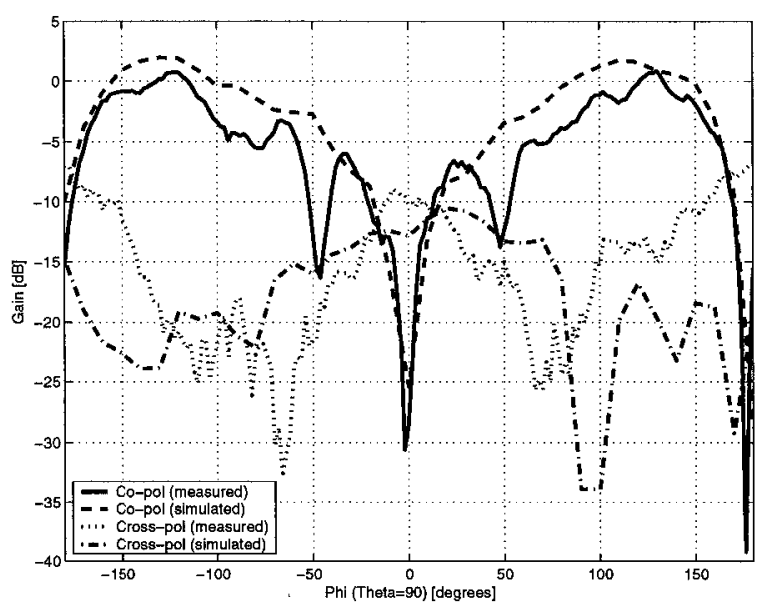

Figure 5.23: Measured azimuthal patterns for FR-4 PGP-TM at $6 \mathrm{GHz}$

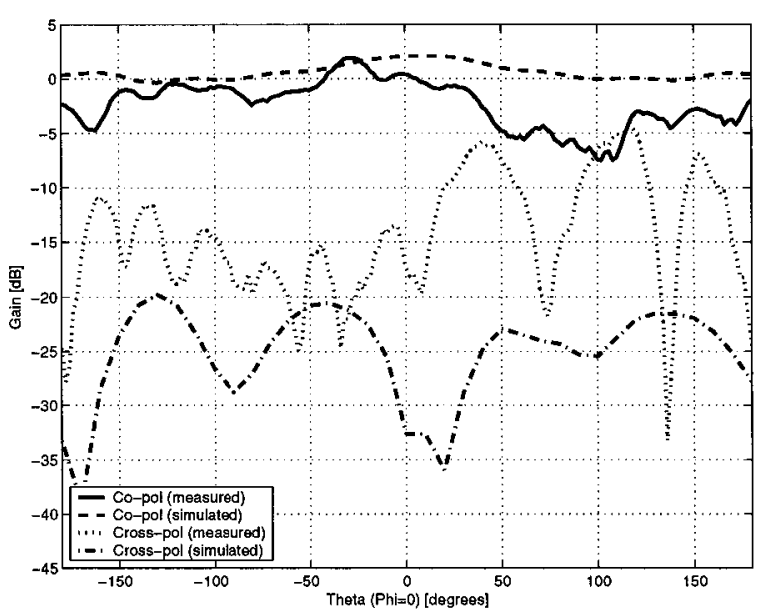

Figure 5.22: Measured elevation patterns for FR-4 PGP-TM at $4 \mathrm{GHz}$

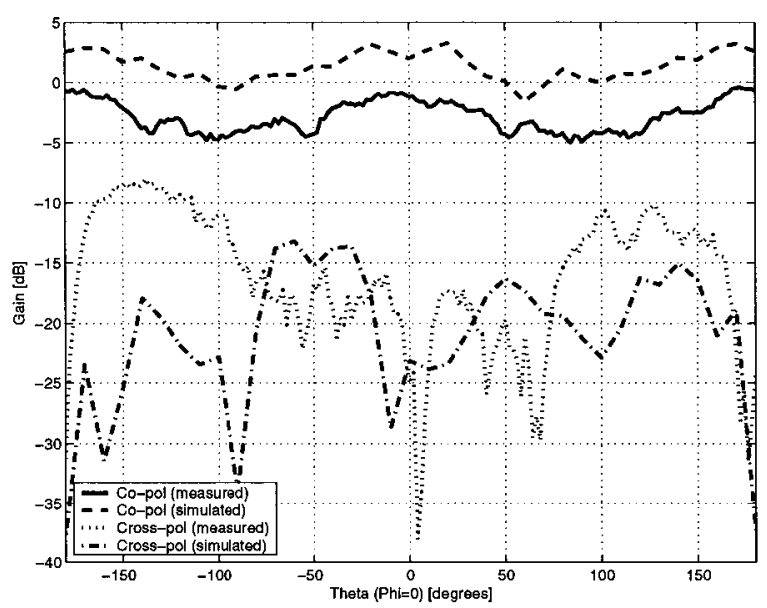

Figure 5.24: Measured elevation patterns for FR-4 PGP-TM at $6 \mathrm{GHz}$ 
amplitude observed was $1.75 \mathrm{mV}$. Due to this large attenuation, noise is more apparent in the received signal than in the AVA case.

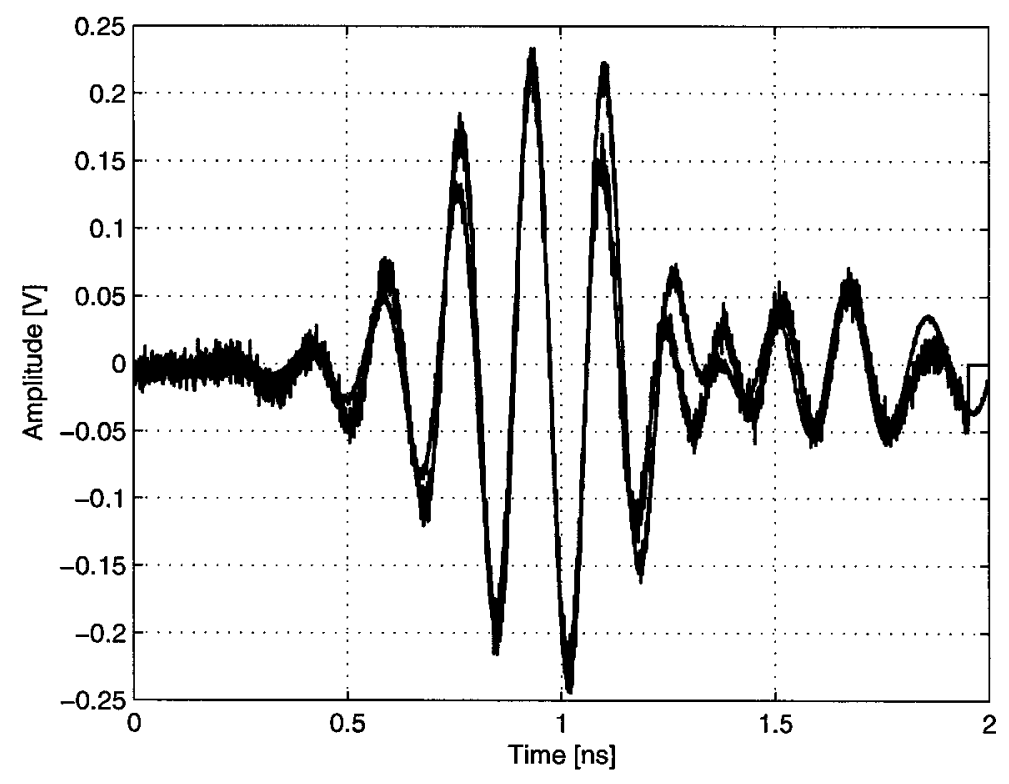

Figure 5.25: Measured FR-4 based PGP-TM time domain response

The results for this design demonstrate several important advantages compared to the FR-4 based AVA, such as more compact size, lower-cost, omni-directional radiation patterns and greater planar circuit integration possibilities. Furthermore, if a return loss that goes slightly above $-10 \mathrm{~dB}$ from $7 \mathrm{GHz}$ to $8 \mathrm{GHz}$ can be tolerated, then this antenna can satisfy the entire UWB bandwidth of $3.1 \mathrm{GHz}$ to $10.6 \mathrm{GHz}$.

\subsection{LTCC-based Antipodal Vivaldi}

The FR-4 based AVA was shown to provide very good performance in both the frequency and time domain with the notable exception of high cross-polarization levels at $8 \mathrm{GHz}$. Beyond the obvious packaging advantages, the LTCC version of the AVA, shown in Figure 
5.26, was developed to investigate what performance advantages the LTCC process could provide compared to the standard FR-4 implementation. The first results obtained were



Figure 5.26: LTCC-based AVA

for return loss, shown in Figure 5.27. The agreement between HFSS and the measured results is very good. The measurements indicate an impedance bandwidth from $6.65 \mathrm{GHz}$ to $10 \mathrm{GHz}$. As a consequence of the size constraint, the low frequency limit is $350 \mathrm{MHz}$ higher than that required by the DS-UWB group.



Figure 5.27: Measured $\left|S_{11}\right|$ of LTCC-based 
The measured $S_{21}$ results, shown in Figure 5.28, of a two LTCC-based AVA system show a very stable magnitude variation. The variation is within $5 \mathrm{~dB}$ in the operating band of the antenna when a line of best fit, whose equation is $y=1.9 x-46$, is used. As expected, the magnitude shows an increasing trend with frequency of almost $20 \mathrm{~dB}$ per decade. The phase shows very good linearity in the entire frequency range from $1 \mathrm{GHz}$ to $10 \mathrm{GHz}$. This leads to a very stable group delay that has a 0.5 ns variation when a $7^{\text {th }}$ degree polynomial is fitted to the data.
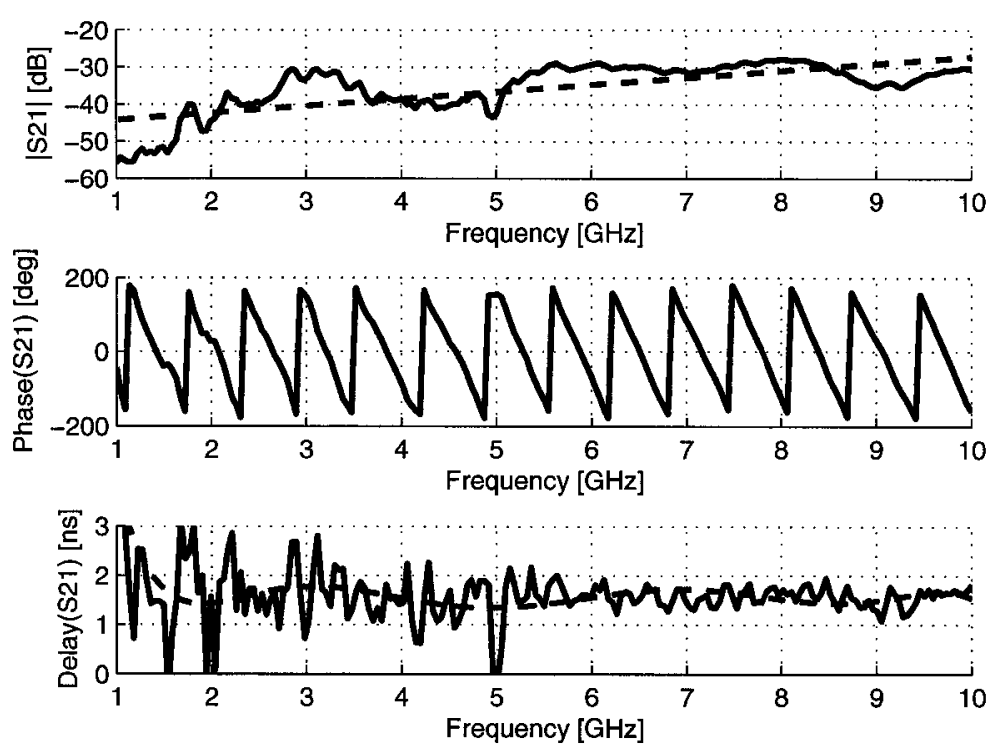

Figure 5.28: Measured $S_{21}$ of LTCC-based AVA

Radiation pattern cuts at $6 \mathrm{GHz}$ and $8 \mathrm{GHz}$ in the two principal planes are shown in Figures 5.29 to 5.32 . In all cases the agreement between the simulated and measured radiation patterns is very good. The radiation pattern is stable and shows no degradation at $8 \mathrm{GHz}$ as was visible in the FR-4 implementation of this antenna. The plots indicate 


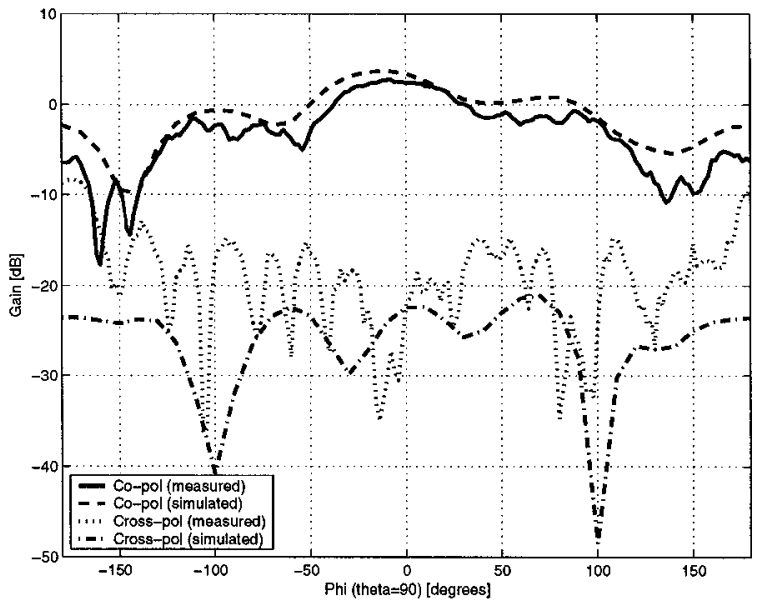

Figure 5.29: Measured azimuthal patterns for LTCC AVA at $6 \mathrm{GHz}$

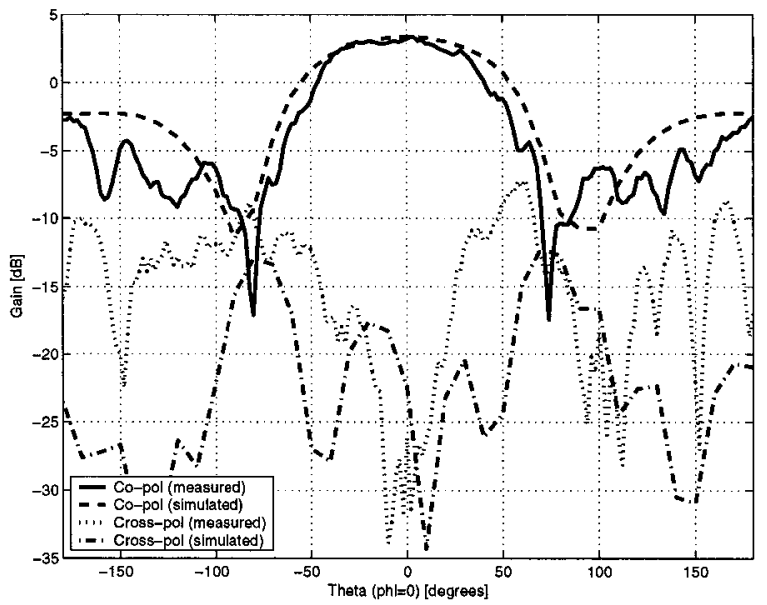

Figure 5.30: Measured elevation patterns for LTCC AVA at $6 \mathrm{GHz}$

a boresight gain of $2.76 \mathrm{~dB}$ at $6 \mathrm{GHz}$ and $5.1 \mathrm{~dB}$ at $8 \mathrm{GHz}$. For the most part, the crosspolarized levels are $20 \mathrm{~dB}$ below the co-polarized levels and are noticeably better than what was measured for the FR-4 version of this antenna. These results show that, along with the small increase in gain, the LTCC substrate provides for much better high frequency radiation performance than the FR-4 substrate. A summary of the simulated and

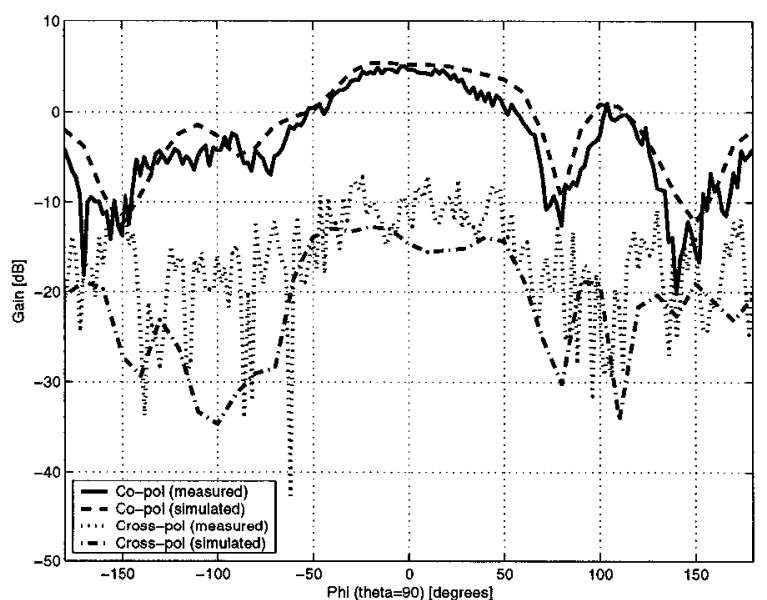

Figure 5.31: Measured azimuthal patterns for LTCC AVA at $8 \mathrm{GHz}$

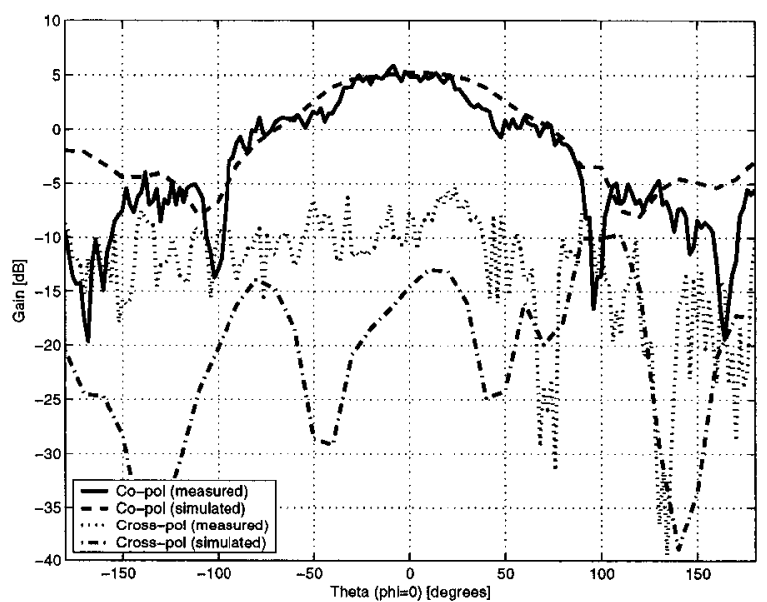

Figure 5.32: Measured elevation patterns for LTCC AVA at $8 \mathrm{GHz}$

measured radiation parameters is provided in Table 5.2. 
Table 5.2: LTCC-based AVA radiation pattern results comparison

\begin{tabular}{|c|c|c|c|c|}
\hline & \multicolumn{2}{|c|}{$6 \mathrm{GHz}$} & \multicolumn{2}{c|}{$8 \mathrm{GHz}$} \\
\cline { 2 - 5 } & Simulated & Measured & Simulated & Measured \\
\hline HPBW (deg) & 180 & 150 & 105 & 100 \\
Gain (dB) & 3.69 & 2.76 & 5.4 & 5.1 \\
FBR & 5.97 & 8.85 & 7.4 & 9.3 \\
Cross-pol (dB) & -25 & -20 & -20 & -15 \\
\hline
\end{tabular}

The excellent $S_{21}$ results for this antenna would indicate good performance in the time domain. Figure 5.33 confirms this supposition. The response in the time domain

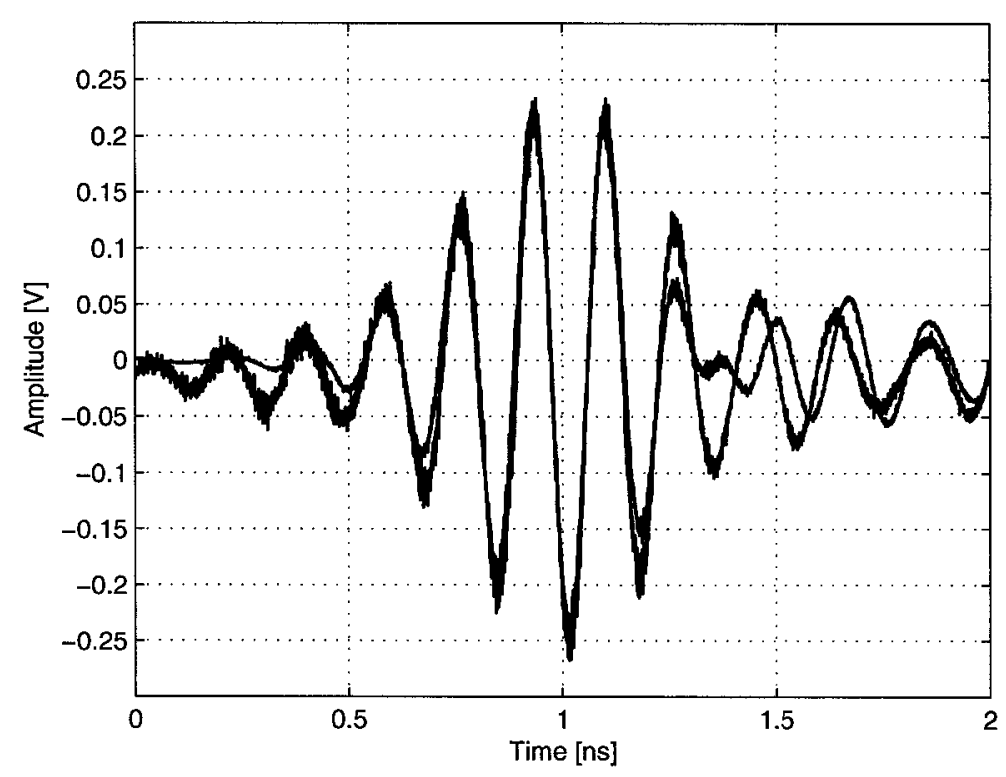

Figure 5.33: Measured LTCC-based AVA time domain response

is very well matched to the ideal case and confirms that frequency domain parameters such as linear phase and a small variation in the group delay indicate good time domain performance. The peak amplitude of the received pulse is $3.44 \mathrm{mV}$. This value cannot be directly compared to the $7.1 \mathrm{mV}$ peak of the FR-4 AVA since that antenna has an 
impedance bandwidth that is $2.65 \mathrm{GHz}$ greater and therefore has the ability to accept more energy.

This design shows that compact Vivaldi antennas can be made to operate at relatively low frequencies and be combined with planar integrated circuits.

\subsection{LTCC-based Triangular Monopole with Partial Ground Plane}

A picture of the LTCC-based PGP-TM, clearly displaying the microchip cavity, is shown

in Figure 5.34. The darker metal areas indicate where silver paste was used to avoid substrate warping. For the same reason, the microchip cavity floor has a meshed ground plane. The return loss measurement shows a total impedance bandwidth of $2.96 \mathrm{GHz}$ from

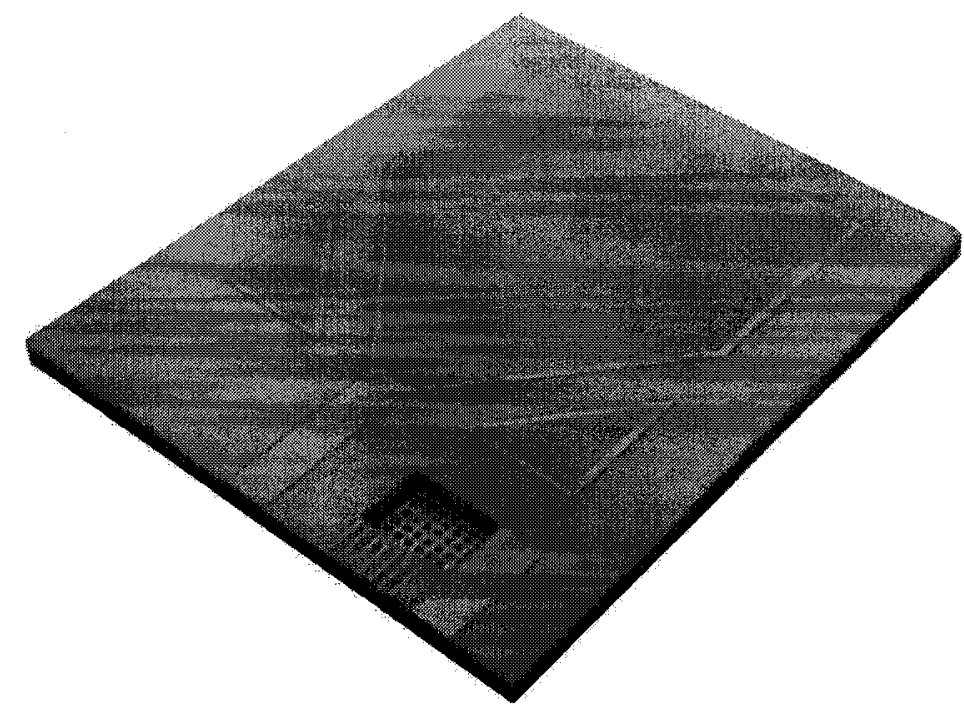

Figure 5.34: LTCC-based PGP-TM

$3.57 \mathrm{GHz}$ to $6.53 \mathrm{GHz}$. The agreement with the simulated results is good. However, HFSS underestimates the lower bound of the impedance bandwidth by approximately $1 \mathrm{GHz}$.

Figure 5.36 displays the magnitude and phase of $S_{21}$ along with group delay. Lines of 


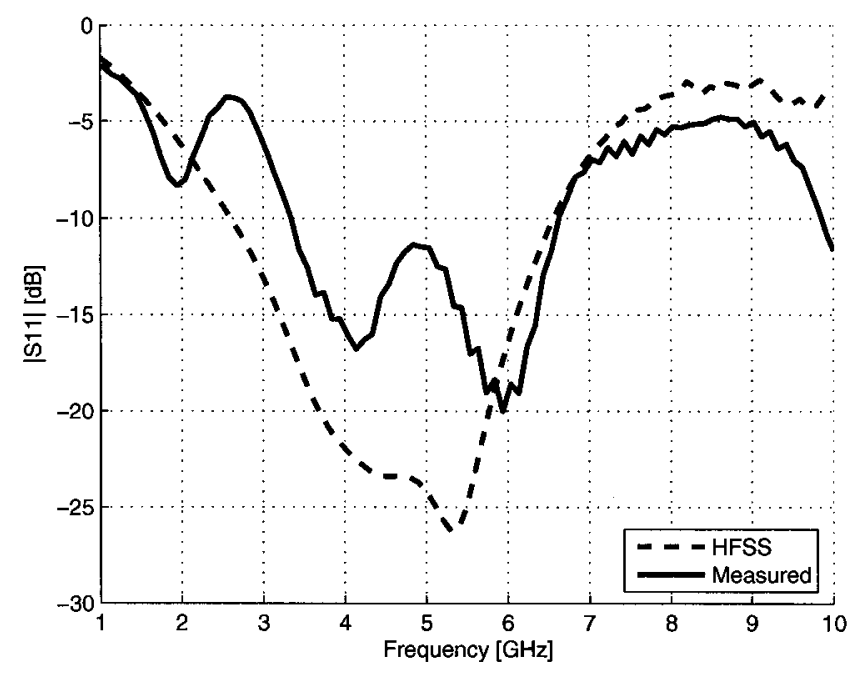

Figure 5.35: Measured $\left|S_{11}\right|$ for LTCC-based PGP-TM

best fit are included to show the best prediction for the variation of $\left|S_{21}\right|$ and group delay with frequency. In the antenna's operating band, the magnitude of $S_{21}$ shows a variation


Figure 5.36: Measured $S_{21}$ for LTCC-based PGP-TM

of $7 \mathrm{~dB}$ when a line of best fit, with equation $y=1.8 x-30$, is applied. The trend with frequency is decreasing at about $20 \mathrm{~dB}$ per decade and agrees with the expected result 
discussed in section 2.6.2 and those obtained for the FR-4 based PGP-TM. The phase of $S_{21}$ is linear beyond $4.5 \mathrm{GHz}$. Even with some initial instability before $4 \mathrm{GHz}$, the group delay stays within a variation of $1.8 \mathrm{~ns}$ in the operating band of the antenna when a $7^{\text {th }}$ degree polynomial is fitted to the data.

Radiation pattern cuts at $4 \mathrm{GHz}$ and $6 \mathrm{GHz}$ in the two principal planes are shown in Figures 5.37 to 5.40. They indicate monopole-like patterns with omni-directional radiation in elevation and gain of approximately $0 \mathrm{~dB}$. The agreement between the simulated and

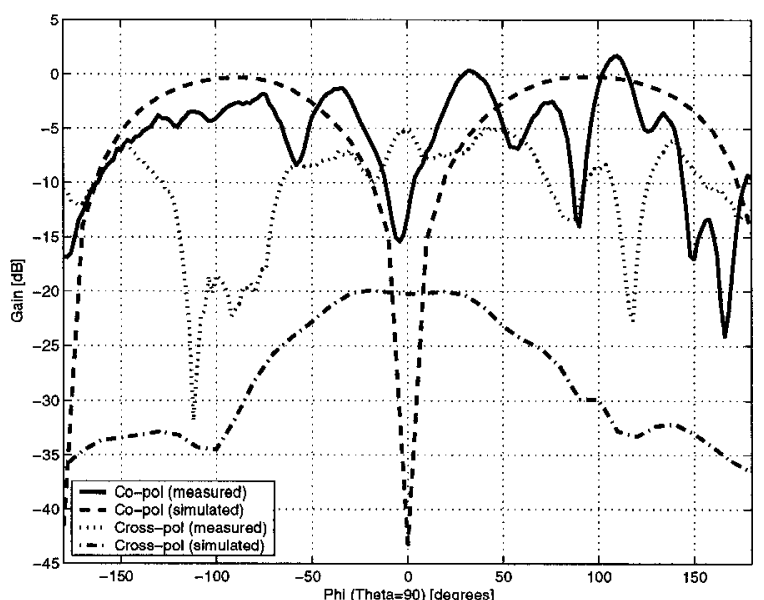

Figure 5.37: Measured azimuthal patterns for LTCC PGP-TM at $4 \mathrm{GHz}$



Figure 5.38: Measured elevation patterns for LTCC PGP-TM at $4 \mathrm{GHz}$

measured radiation patterns is good except for notably underestimated cross-polarization levels at $4 \mathrm{GHz}$. Compared to the radiation patterns of the FR-4 based PGP-TM, this antenna's patterns are more stable and closer to the ideal monopole shape. The radiation performance of this antenna improves at $6 \mathrm{GHz}$ where the cross-polarized levels are, in general, below $-15 \mathrm{~dB}$.

The response in the time domain is very good and is shown in Figure 5.41. The peak amplitude observed in the received pulse is $1.88 \mathrm{mV}$, which $0.13 \mathrm{mV}$ or $7 \%$ higher than in 




Figure 5.39: Measured azimuthal patterns for LTCC PGP-TM at $6 \mathrm{GHz}$

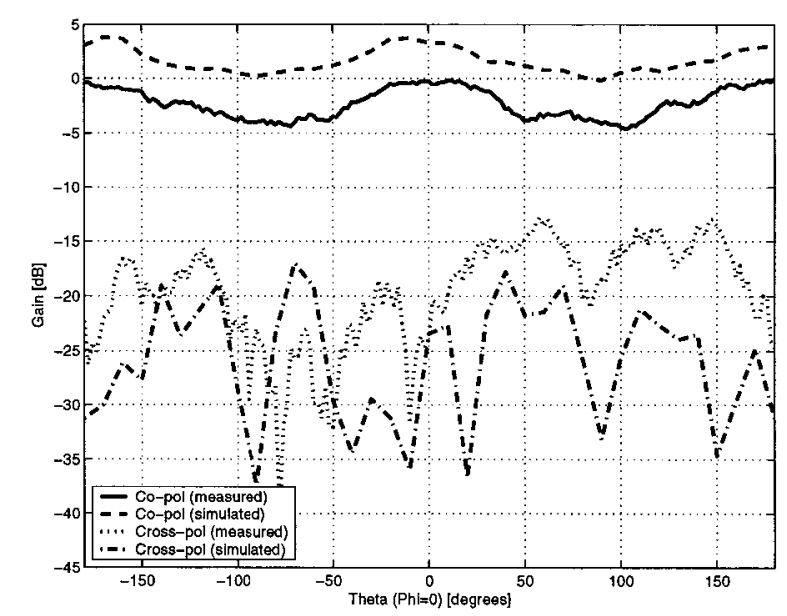

Figure 5.40: Measured elevation patterns for LTCC PGP-TM at $6 \mathrm{GHz}$

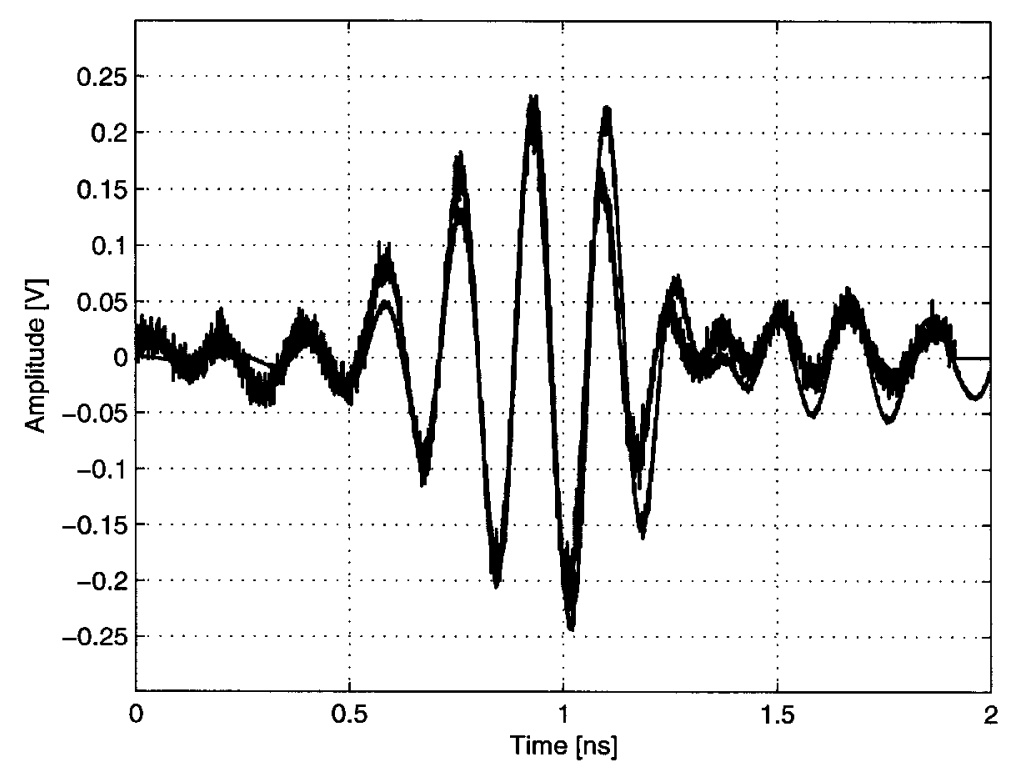

Figure 5.41: Measured LTCC-based PGP-TM time domain response 
the FR-4 implementation of this antenna. This is significant since the FR-4 PGP-TM has a $1.94 \mathrm{GHz}$ larger impedance bandwidth and therefore can accept more energy than its LTCC counterpart. This helps to create a reduction in the noise present in the received signal. Since the gain is unchanged, it may be that the lower loss LTCC substrate has increased the efficiency of this PGP-TM.

Compared to the FR-4 implementation, the LTCC-based PGP-TM shows important advantages such as more stable radiation patterns, higher average gain, lower crosspolarization levels, increased efficiency, improved response in the time domain and smaller size.

\subsection{Measurement Summary and Discussion}

Five of the antenna designs presented in Chapter 4 are manufactured and tested in both the frequency and time domain. Namely, an OGP-TM and FR-4 and LTCC implementations of the PGP-TM and AVA. A patch antenna is tested for comparative purposes. The simulation results are in good agreement with the measured values. The results are compared to determine what advantages can be gained by using an LTCC substrate as apposed to FR-4 and which antenna is the best candidate for UWB applications.

First, a $5 \mathrm{GHz}$ patch antenna is used to determine the response in the time domain characteristic of a very narrowband antenna. As expected, the received pulse suffered from significant distortion and would not be recognized as a valid UWB signal. Next, an OGP-TM antenna with a more extensive bandwidth of approximately $1.14 \mathrm{GHz}$ from 4.5 $\mathrm{GHz}$ to $5.64 \mathrm{GHz}$ is measured in the time domain. Its response shows less distortion 
than in the case of the patch but the received pulse is nonetheless significantly changed from the original transmitted signal. This antenna shows that broadband antennas can be designed using very high permittivity substrates of $\varepsilon_{r}=68$. A disadvantage of this antenna is its non planar topology, which makes it occupy more volume than the other planar antennas presented in this work. This exercise makes it clear that a wider band antenna is necessary for UWB applications.

The AVA is an inherently broadband antenna topology, which is also known to have good time domain performance. The measured results for a version of this antenna built on an FR-4 substrate show an impedance bandwidth from $4 \mathrm{GHz}$ to $10 \mathrm{GHz}$. The results for a two antenna system show that the $\left|S_{21}\right|$ is not increasing at the rate expected, which may be due to large substrate losses at higher frequencies. Although, the received pulse, in the time domain, shows very little distortion. The drawbacks to this design are its relatively large size and poor radiation performance above $8 \mathrm{GHz}$, again due to the large losses of the FR-4 substrate.

To alleviate the problems encountered with the FR-4 based AVA, an LTCC-based AVA is measured. The s-parameter results show an impedance bandwidth from $6.65 \mathrm{GHz}$ to $10 \mathrm{GHz}$ and an increasing system $\left|S_{21}\right|$ of $19 \mathrm{~dB}$ per decade that validates the theories discussed in section 2.6.2. This is the only antenna in this study that provides a suitable cancelation for path loss. The antenna has excellent time domain performance along with very well maintained radiation patterns at $8 \mathrm{GHz}$. This antenna displays improved time domain and radiation performance, while occupying $55 \%$ less area relative to the FR-4 based AVA. Furthermore, this antenna is inherently suitable for a UWB link due to its 
increasing $\left|S_{21}\right|$ with almost $20 \mathrm{~dB}$ per decade slope.

A FR-4 based PGP-TM antenna intended for applications where an omni-directional radiation pattern is required is measured next. The s-parameter results show an impedance bandwidth from $3.47 \mathrm{GHz}$ to $6.82 \mathrm{GHz}$ and a decreasing system $\left|S_{21}\right|$ of $18 \mathrm{~dB}$ per decade that agrees with the theories discussed in section 2.6.2 regarding an a UWB link composed of two omni-directional antennas. Radiation measurements indicate monopolelike patterns with omni-directional radiation in elevation and gain of approximately $0 \mathrm{~dB}$. The pattern remains stable over the operating bandwidth. The time domain response is excellent. As with the FR-4 based AVA, the high substrate losses above $7 \mathrm{GHz}$ are a drawback.

The LTCC-based equivalent of the PGP-TM antenna achieves similar or superior performance figures to its FR-4 based counterpart, while occupying $29 \%$ less area. Impedance bandwidth is measured to be from $3.57 \mathrm{GHz}$ to $6.53 \mathrm{GHz}$. Radiation performance is improved, especially the cross-polarization levels at $6 \mathrm{GHz}$. Also, the received time domain signal is slightly better maintained than in the case of the FR-4 based PGP-TM.

The measurement of an antenna system's $S_{21}$ is a good indicator of its time domain performance. A comparison of the trend of $\left|S_{21}\right|$ with respect to frequency for the four antenna designs discussed above is shown in Figure 5.42.

As expected, the Vivaldi antennas display a higher $\left|S_{21}\right|$ than the PGP-TM antennas. However, the relatively larger size and directional nature of the Vivaldi antennas may be a tradeoff that is difficult to tolerate. The antennas implemented with LTCC prove to be more compact and better performing, especially with regards to their radiation 


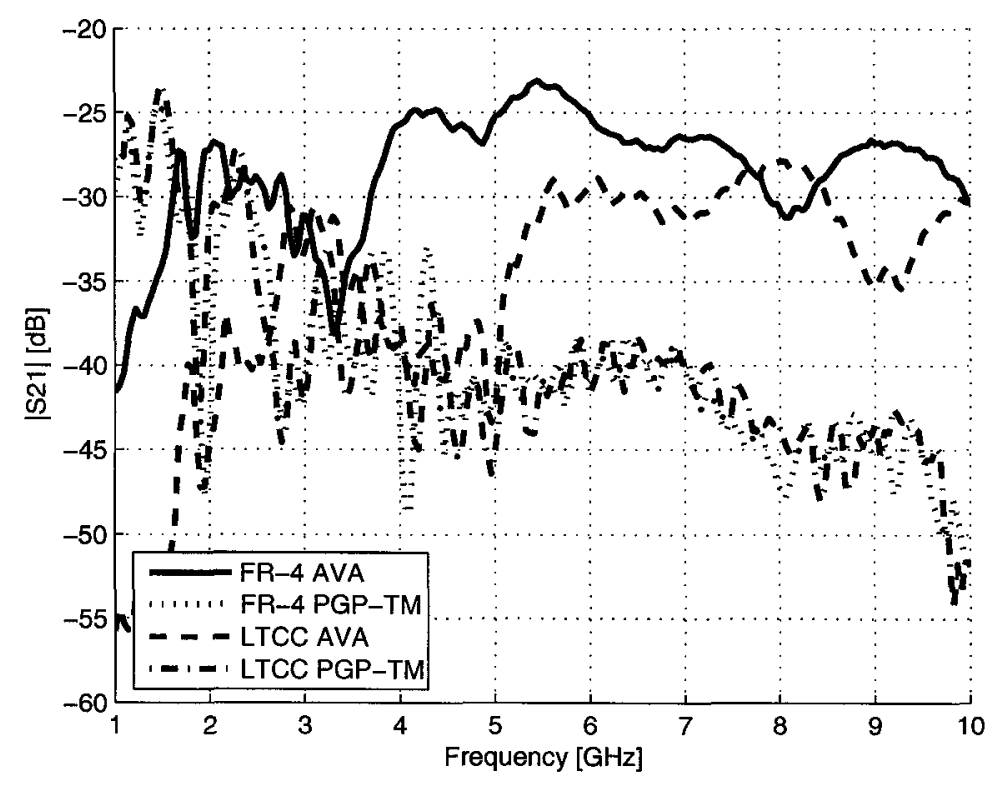

Figure 5.42: System $\left|S_{21}\right|$ trend with frequency

characteristics. In the case of the FR-4 based AVA, the substrate causes significant pattern degradation at $8 \mathrm{GHz}$ and a leveling off of the $\left|S_{21}\right|$ curve. The LTCC based AVA showed no such degradation or leveling off.

Table 5.3 displays a summary of the measured parameters for the four antenna designs based on FR-4 and LTCC substrates.

Although all the antennas listed in Table 5.3 are suitable for UWB applications, the measurement results reveal that the LTCC based antennas are superior to their FR-4 counterparts in terms of performance, size and packaging possibilities. 
Table 5.3: UWB Antenna Measurement Summary

\begin{tabular}{|c|c|c|c|c|}
\hline Antenna & FR-4 PGP-TM & LTCC PGP-TM & FR-4 AVA & LTCC AVA \\
\hline Dimensions $[\mathrm{mm}]$ & $35 \times 30$ & $30 \times 25$ & $70 \times 40$ & $50 \times 25$ \\
\hline Substrate & FR-4 & Ferro A6 & FR-4 & Ferro A6 \\
\hline Metal & Gold & Silver & Gold & Silver \\
\hline $\begin{array}{l}\text { Impedance } \quad \mathrm{BW} \\
{[\mathrm{GHz}]}\end{array}$ & $3.47-6.82$ & $3.57-6.53$ & $4-10^{1}$ & $6.65-10$ \\
\hline Radiation pattern & omni & omni & directional & directional \\
\hline $\begin{array}{l}\text { Cross-pol levels at } \\
\text { peak gain }[\mathrm{dB}]\end{array}$ & 15 & 15 & 17 & 25 \\
\hline $\begin{array}{l}\text { Low frequency gain } \\
{[\mathrm{dB}]}\end{array}$ & 0 & 0 & 4.32 & 2.76 \\
\hline $\begin{array}{l}\text { High frequency gain } \\
{[\mathrm{dB}]}\end{array}$ & 0 & 0 & 3 & 5.1 \\
\hline $\begin{array}{l}\left|S_{21}\right| \text { slope with fre- } \\
\text { quency }[\mathrm{dB} / \mathrm{dec}]\end{array}$ & -18 & -18 & 6 & 19 \\
\hline $\begin{array}{l}\text { Group delay varia- } \\
\text { tion over operating } \\
\text { BW [ns] }\end{array}$ & 2 & 1.8 & 0.7 & 0.5 \\
\hline $\begin{array}{l}\text { Time domain re- } \\
\text { sponse }\end{array}$ & Excellent & Excellent & Good & Excellent \\
\hline
\end{tabular}

1: Radiation performance limits practical bandwidth to below $8 \mathrm{GHz}$ 


\section{Chapter 6}

\section{Conclusions and Future Work}

\subsection{Conclusions}

The feasibility of designing and fabricating small, low cost, UWB compliant antennas has been investigated in this thesis. The DS-UWB implementation was selected as the target application. Requirements, and practical design guidelines for UWB antennas were formulated before the design and full characterization of a number of novel antennas was undertaken. The most important requirements are that a candidate UWB antenna have sufficient impedance bandwidth and a system $S_{21}$ that has a flat magnitude and linear phase. These requirements are most likely met by antennas that support traveling waves or that have a low $\mathrm{Q}$. Most often, these antennas incorporate tapers or rounded edges to give surface currents a smooth path to follow.

A FDTD based electromagnetic simulator was developed as an in-house tool to evaluate the time domain performance of the antennas under arbitrary pulse shape excitations. As a proven commercial FEM-based solver, HFSS was also used to investigate several fundamental parameters of UWB antenna operation, including the effects of geometry, substrate permittivity, size, parasitic elements and different ground configurations 
on frequency domain performance. The results of the FDTD simulator and HFSS were compared to the measured results and were found to be in good agreement.

Based on the introduced guidelines, AVA and PGP-TM antenna topologies were selected. The designs were optimized and realized in both FR-4 and LTCC substrates. The AVA constructed on an FR-4 substrate has a measured impedance bandwidth of $6 \mathrm{GHz}$, from $4 \mathrm{GHz}$ to $10 \mathrm{GHz}$. Radiation pattern measurements, performed in an anechoic chamber, revealed a directional pattern with a gain of $4.32 \mathrm{~dB}$ at $6 \mathrm{GHz}$. However, at $8 \mathrm{GHz}$ pattern stability degraded because of the limitations of the FR-4 substrate at high frequencies. The LTCC version of the AVA shows no such deficiencies while occupying $55 \%$ less area than its FR-4 counterpart. An increasing system $S_{21}$ slope of $19 \mathrm{~dB}$ per decade is observed - making this the only antenna in this study that provides a suitable cancelation for path loss. This antenna also incorporates a novel circuit feature to accommodate transceiver electronics. The impedance bandwidth for this antenna was $3.35 \mathrm{GHz}$, from $6.65 \mathrm{GHz}$ to $10 \mathrm{GHz}$. This makes it suitable for use in the upper band of the DS-UWB implementation. Gain was measured to be $5 \mathrm{~dB}$ at $8 \mathrm{GHz}$. A UWB pulse was transmitted and received with very little added distortion in an antenna system utilizing these AVAs.

Omni-directional antennas were also designed and fully characterized. Beginning with an OGP-TM that has a bandwidth of $1.14 \mathrm{GHz}$ from $4.5 \mathrm{GHz}$ to $5.64 \mathrm{GHz}$. This antenna has a gain of $0 \mathrm{~dB}$ and a monopole like pattern. This antenna shows that broadband antennas can be designed using very high permittivity substrates of $\varepsilon_{r}=68$. The characteristics of this antenna make it suitable for many WLAN applications. Planar omni-directional antennas were also investigated. The PGP-TM based on a FR-4 substrate has a return 
loss bandwidth of $3.35 \mathrm{GHz}$ or $4.9 \mathrm{GHz}$ when a small peak above $-10 \mathrm{~dB}$ at $8 \mathrm{GHz}$ is neglected - making this antenna nearly capable of spanning the full DS-UWB bandwidth. The expected decreasing system $\left|S_{21}\right|$ of almost $20 \mathrm{~dB}$ per decade is observed. The PGPTM implemented in LTCC occupies $29 \%$ less area than its FR-4 counterpart while also providing better radiation and time domain performance. The impedance bandwidth is measured to be from $3.57 \mathrm{GHz}$ to $6.53 \mathrm{GHz}$ - making this antenna suitable for use in the lower band of the DS-UWB implementation. The slope of the system $\left|S_{21}\right|$ is the same as with the FR-4 PGP-TM. Both substrate implementations of the PGP-TM antenna utilize novel parasitic tuning elements for optimized performance.

By validating the newly formulated requirements and guidelines, this thesis has laid the groundwork for the comprehensive design of UWB antennas. Implementing antennas in LTCC has proven to provide many advantages relative to other substrate implementations. The stable material properties and low loss of LTCC make it ideal for UWB antenna applications. Furthermore, because it is also a packaging technology, transceiver electronics can be easily integrated with the antennas making for a very compact module. The LTCC AVA, measuring only $50 \mathrm{~mm} \times 25 \mathrm{~mm} \times 1.2 \mathrm{~mm}$, is the first demonstration of a compact antipodal Vivaldi antenna with circuit integration possibilities suitable for UWB applications. The LTCC PGP-TM, measuring only $30 \mathrm{~mm} \times 25 \mathrm{~mm} \times 1.2 \mathrm{~mm}$, is the smallest demonstration of a monopole antenna with circuit integration possibilities suitable for UWB applications. 


\subsection{Main Contributions}

The main contribution of this thesis is an increased understanding of the tradeoffs between antenna design and transceiver complexity. Comprehensive designs of UWB antennas can be made by utilizing the requirements, guidelines and results contained in this thesis.

The other contributions of this thesis are as follows:

- The first demonstration of a compact LTCC AVA that has a system $\left|S_{21}\right|$ that provides a cancelation for path loss while being appropriate for UWB applications. Using this antenna in a UWB link can reduce the complexity of the transceiver by eliminating the need for a variable gain amplifier (to maintain a flat system $\left|S_{21}\right|$ ).

- The smallest example of an LTCC PGP-TM antenna suitable for UWB.

- Novel use of parasitic elements for the optimization of compact and wideband PGP-TM antennas.

- The first demonstration of UWB LTCC antennas with a novel circuit feature that can accommodate transceiver electronics.

- The first report of a compact and broadband OGP-TM antenna implemented on a $\varepsilon_{r}=68$ LTCC substrate.

Finally, the implications of designing antennas in an advanced packaging technology like LTCC cannot be overlooked. As discussed earlier, the use of LTCC leads to several advantages that would not be attainable if the antennas were implemented in FR-4 or Duroid. 


\subsection{Future Work}

The FDTD simulator developed in this thesis proved to be accurate. However, additional features could be implemented. The three main improvements that can be made are:

- Replacing the current MUR absorbing boundary condition with a more effective PML boundary.

- Implementing the near-to-far-field transformation to allow for radiation pattern generation.

- Developing a graphical user interface to simplify the use of the software.

Work that is a logical next step is the development of a microchip circuit that can be combined with the LTCC antennas to form a complete communications module. Finally, developing a UWB pulse that better conforms to the spectrum mask (only a $2 \mathrm{GHz}$ bandwidth was used in this thesis) and a more rigorous method for assessing the transmission characteristics in the time domain represent pertinent future work. 


\section{Bibliography}

[1] D. Barras, F. Ellinger, H. Jackel, and W. Hirt, "A low supply voltage SiGe LNA for ultra-wideband frontends," IEEE Microwave and Wireless Components Letters, vol. 14, pp. 469-471, October 2004.

[2] A. Bevilacqua and A. M. Niknejad, "An ultra-wideband CMOS low-noise amplifier for 3.1-10.6 GHz wireless receivers," IEEE Journal of Solid-State Circuits, vol. 39, pp. 2259-2268, December 2004.

[3] M. D. Tsai and H. Wang, "A $0.3-25 \mathrm{GHz}$ ultra-wideband mixer using commercial 0.18- $\mu \mathrm{m}$ CMOS technology," IEEE Microwave and Wireless Components Letters, vol. 14, pp. 522-524, November 2004.

[4] F. S. Lee, D. D. Wentzloff, and A. P. Chandrakasan, "An ultra-wideband baseband front-end," in IEEE Radio Frequency Integrated Circuits (RFIC) Symposium, pp. 493-496, June 2004.

[5] G. Lu, P. Spasojevic, and L. Greenstein, "Anntena and pulse designs for meeting UWB spectrum density requirements," in Ultra Wideband Systems and Technologies, IEEE Conference on, pp. 162-166, 16-19 November 2003. 
[6] D. Lamensdorf and L. Susman, "Baseband-pulse-antenna techniques," IEEE Antennas and Propagation Magazine, vol. 36, February 1994.

[7] X. H. Wu, Z. N. Chen, and M. Chia, "Note on antenna design in UWB wireless communication systems," in Ultra Wideband Systems and Technologies, IEEE Conference on, pp. 503-507, 16-19 November 2003.

[8] G. R. Aillo and G. D. Rogerson, "Ultra-wideband wireless systems," IEEE microwave magazine, pp. 36-47, June 2003.

[9] R. J. Fontana, "A brief history of UWB communications." http://www.multispectral.com/history.html.

[10] "Part 15 - radio frequency devices," tech. rep., Federal Communications Commission, [online] http://www.fcc.gov/oet/info/rules/part15/part15_1_26_05.pdf, 2005.

[11] R. Fisher, R. Kohno, M. McLaughlin, and M. Welborn, "DS-UWB physical layer submission to 802.15 task group 3a," July 2004.

[12] D. M. Pozar, Microwave Engineering, p. 636. John Wiley and Sons, Inc., third edition ed., 2005.

[13] A. Petosa, Antennas and Arrays Course Notes, pp. 1-30. Carleton University, 2002.

[14] W. L. Stutzman and G. A. Thiele, Antenna Theory And Design, pp. 37-52. John Wiley and Sons, Inc., second edition ed., 1998. 
[15] K. Kautio, "LTCC tutorial," tech. rep., VTT [online] www.vtt.fi/ele/research/ope/ltcc_tutorial.htm.

[16] Y. Lin, C. Liu, K. Li, and C. Chen, "Design of an LTCC tri-band transceiver module for GPRS mobile applications," Microwave Theory and Techniques, IEEE Transactions on, vol. 52, pp. 2718 - 2724, December 2004.

[17] L. Pergola, R. Vahldieck, U. Gobel, and P. Nuchter, "An LTCC-based 5-6 GHz receiver with integrated antenna," in Wireless Technology, 7th European Conference on, pp. $165-168$, October 2002.

[18] H. G. Schantz, "Introduction to ultra-wideband antennas," in Ultra Wideband Systems and Technologies, IEEE Conference on, pp. 1-9, 16-19 November 2003.

[19] S. H. Choi, J. K. Park, S. K. Kim, and J. Y. Park, "A new ultra-wideband antenna for UWB applications," Microwave and Optical Letters, pp. 399-401, 2004.

[20] C.Ying and Y.Zhang, "Integration of ultra-wideband slot antenna on LTCC substrate," Electronics Letters, vol. 40, pp. 645-646, May 2004.

[21] J. Liang, C. Chiau, X. Chen, and C. Parini, "Printed circular disc monopole antenna for ultra-wideband applications," in Electronics Letters, vol. 40, pp. 1246- 1247, September 2005.

[22] Q. Ye, "Time domain response of ultra wideband dipole antennas," in Antem/URSI Conference on Antenna Technology and Applied Electromagnetics, (Ottawa, Canada), pp. 661-664, July 2004. 
[23] W. S. Cho, M. Kanda, H. J. Hwang, and M. W. Howard, "A disk-loaded thick cylindrical dipole antenna for validation of an emc test site from 30 to $300 \mathrm{MHz}$," Electromagnetic Compatibility, IEEE Transactions on, vol. 42, pp. 172-180, May 2000.

[24] M. Fan, X. Zhang, and Z. Feng, "An improved type slot-stacked patch antenna," in Microwave Conference, Asia Pacific, vol. 3, pp. 742 - 745, December 1999.

[25] E. Tentzeris, R. Li, K. Lim, M. Maeng, E. Tsai, G. DeJean, and J. Laskar, "Design of compact stacked-patch antennas on LTCC technology for wireless communication applications," in Antennas and Propagation Society International Symposium, IEEE, vol. 2, pp. 500-503, June 2002.

[26] J. McLean, "A re-examination of the fundamental limits on the radiation q of electrically small antennas," Antennas and Propagation, IEEE Transactions on, vol. 44, pp. 672-676, May 1996.

[27] V. Rumsey, Frequency Independant Antennas. New York: Academic Press, 1966.

[28] N. Cohen, R. Hohfield, and D. Moschella, "RFID benefits from fractals," Technology Focus, pp. 14-15, 1996.

[29] C. Puente-Baliarda, J. Romeu, R. Pous, and A. Cardama, "On the behavior of the sierpinski multiband fractal antenna," Antennas and Propagation, IEEE Transactions on, vol. 46, no. 4, pp. 517-524, 1998. 
[30] J. P. Gianvittorio and Y. Rahmat-Samii, "Fractal antennas: A novel antenna miniaturization technique, and applications," Antennas and Propagation, IEEE Transactions on, vol. 44, no. 1, pp. 20-36, 2002.

[31] E. Gazit, "Improved design of the vivaldi antenna," IEE Proceedings Microwaves, Antennas and Propagation, vol. 135, no. 2, pp. 89-92, 1988.

[32] D. Sullivan, Electromagnetic Simulation Using the FDTD Method. IEEE Press, 2004.

[33] D. Sheen, S. Ali, M. Abouzahra, and J. Kong, "Application of the three-dimensional finite-difference time domain method to the analysis of planar microstrip circuits," Microwave Theory and Techniques, IEEE Transactions on, vol. 38, pp. 103-110, July 1990.

[34] M. Barari, A. Tavakoli, and R. Moeini, "Analysis of finite vivaldi antenna arrays using a FDTD method," in 3rd International Conference on Microwave and Millimeter Wave Technology Proceedings, pp. 642 - 645, August 2002.

[35] K. Yee, "Numerical solution of initial boundary value problems involving maxwell's equations in isotropic media," Antennas and Propagation, IEEE Transactions on, vol. 14, pp. 302-307, 1966.

[36] A. Taflove and S. Hagness, Computational Electrodynamics the FDTD Method. Boston: Artech House, 2 ed., 2000. 
[37] G. Mur, "Absorbing boundary conditions for the finite-difference approximation of the time domain electromagnetic-field equations," Electromagnetic Compatibility, IEEE Transactions on, vol. EMC-23, pp. 377-382, 1981.

[38] L. Godara, Handbook of Antennas in Wireless Communications. CRC Press, 2002.

[39] P. Gibson, "The vivaldi aerial," in 9th European Microwave Conference, 1979.

[40] V. Mikhnev and P. Vainikainen, "Wideband tapered-slot antenna with corrugated edges for GPR applications," 33rd European Microwave Conference, vol. 2, pp. 727729, 2003.

[41] W. Sorgel, C. Waldschmidt, and W. Wiesbeck, "Transient responses of a vivaldi antenna and logarithmic periodic dipole array for UWB communication," in IEEE Antennas and Propagation Society International Symposium, vol. 3, pp. 592-595, 2003.

[42] J. Shin and D. H. Schaubert, "A parameter study of stripline-fed vivaldi notchantenna arrays," Antennas and Propagation, IEEE Transactions on, vol. 47, May 1999.

[43] J. Langley, P. Hall, and P. Newman, "Balanced antipodal vivaldi antenna for wide bandwidth phased arrays," in Microwaves, Antennas and Propagation, IEE Proceedings, vol. 143, pp. 97-102, 1996.

[44] J. Noronha, T. Bielwa, C. Anderson, D. Sweeney, S. Licul, and W. Davis, "Designing antennas for UWB systems," Micorwaves and RF Magazine, pp. 53-61, 2003. 
[45] B. Wadell, Transmission Line Design Handbook. Artech House, 1991.

[46] T. K. Sarkar, M. C. Wicks, M. Salazar-Palma, and R. J. Bonneau, Smart Antennas, pp. 11-59. John Wiley and Sons, Inc., 2003.

[47] S. Y. Suh, W. L. Stutzman, and W. A. Davis, "Multi-broadband monopole disc antennas," in Antennas and Propagation Society International Symposium, IEEE, vol. 3, pp. 616-619, 2003.

[48] Z. Chen, M. Chia, and M. Ammann, "Optimization and comparison of broadband monopoles," in Microwaves, Antennas and Propagation, IEE Proceedings, no. 6, pp. 429-435, December 2003.

[49] L. Desclos, M.Madihian, J. Floc'h, and T. Ohsawa, "1.6-6 GHz optimized antennas for wireless LAN applications," in Wireless Applications Digest, IEEE MTT-S Symposium on Technologies for, pp. 39-42, 1997.

[50] O. Lodge, "Electric telegraphy." U.S. Patent 609,514, 1898.

[51] G. Brown and O. Woodward, "Experimentally determined radiation characteristics of conical and triangular antennas," RCA Review, vol. 13, pp. 425-452, 1952.

[52] A. Eldek, A. Elsherbeni, and C. Smith, "Wideband bow-tie slot antenna with tuning stubs," in Proceedings of the IEEE Radar Conference, pp. 583-588, April 2004.

[53] H. Chen, "Microstrip-fed dual-frequency printed triangular monopole," Electronics Letters, vol. 38, June 2002. 
[54] J. S. McLean, H. Foltz, and R. Sutton, "Pattern descriptors for UWB antennas," Antennas and Propagation, IEEE Transactions on, vol. 53, pp. 553-559, January 2005.

[55] D. Ball, P. Charlebois, and W. Lauber, "Ultra-wideband signal sources for interference measurements," in IEEE International Conference on Ultra-Wideband, September 2005. 